\title{
Near-infrared photometry of globular clusters towards the Galactic bulge: observations and photometric metallicity indicators
}

\author{
Roger E. Cohen, ${ }^{1 \star}$ Christian Moni Bidin, ${ }^{2}$ Francesco Mauro, ${ }^{1,3}$ Charles Bonatto ${ }^{4}$ \\ and Douglas Geisler ${ }^{1}$ \\ ${ }^{1}$ Departamento de Astronomía, Universidad de Concepción, Casilla 160-C, Concepción, Chile \\ ${ }^{2}$ Instituto de Astronomía, Universidad Católica del Norte, Av. Angamos 0610, Casilla 1280, Antofagasta, Chile \\ ${ }^{3}$ Instituto Milenio de Astrofísica, Santiago, Chile \\ ${ }^{4}$ Departamento de Astronomia, Universidade Federal do Rio Grande do Sul, Av. Bento Gonçalves 9500 Porto Alegre 91501-970, RS, Brazil
}

Accepted 2016 September 23. Received 2016 September 21; in original form 2015 December 7

\begin{abstract}
We present wide-field $J H K_{S}$ photometry of 16 Galactic globular clusters located towards the Galactic bulge, calibrated on the Two Micron All-Sky Survey photometric system. Differential reddening corrections and statistical field star decontamination are employed for all of these clusters before fitting fiducial sequences to the cluster red giant branches (RGBs). Observed values and uncertainties are reported for several photometric features, including the magnitude of the RGB bump, tip, the horizontal branch (HB) and the slope of the upper RGB. The latest spectroscopically determined chemical abundances are used to build distance- and reddening-independent relations between observed photometric features and cluster metallicity, optimizing the sample size and metallicity baseline of these relations by supplementing our sample with results from the literature. We find that the magnitude difference between the HB and the RGB bump can be used to predict metallicities, in terms of both iron abundance $[\mathrm{Fe} / \mathrm{H}]$ and global metallicity $[\mathrm{M} / \mathrm{H}]$, with a precision of better than $0.1 \mathrm{dex}$ in all three near-IR bandpasses for relatively metal-rich $([\mathrm{M} / \mathrm{H}] \gtrsim-1)$ clusters. Meanwhile, both the slope of the upper RGB and the magnitude difference between the RGB tip and bump are useful metallicity indicators over the entire sampled metallicity range $(-2 \lesssim[M / H] \lesssim 0)$ with a precision of 0.2 dex or better, despite model predictions that the RGB slope may become unreliable at high (near-solar) metallicities. Our results agree with previous calibrations in light of the relevant uncertainties, and we discuss implications for clusters with controversial metallicities as well as directions for further investigation.
\end{abstract}

Key words: globular clusters: general-infrared: stars.

\section{INTRODUCTION}

Galactic globular clusters (GGCs) play a crucial role in constraining stellar evolutionary models as well as Galactic chemical evolution. Recently, many of these clusters have been the subject of largescale photometric surveys using deep, high-resolution multicolour space-based observations (Piotto et al. 2002; Sarajedini et al. 2007; Piotto et al. 2015). However, GGCs located towards the Galactic bulge, despite their importance as the most metal-rich (and in some cases, massive) members of the GGC system, have been generally excluded from these surveys due to severe total and differential extinction at optical wavelengths. For this reason, infrared (IR) wavelengths, where the effects of extinction are greatly reduced

^E-mail: rcohen@ astro-udec.cl
$\left(A_{K} \sim 0.12 A_{V}\right.$; Casagrande \& VandenBerg 2014), are ideal for photometric investigations of such clusters.

The Vista Variables in the Via Lactea (VVV), a European Southern Obseratory (ESO) public survey, has observed a $562 \mathrm{deg}^{2}$ field including the Galactic bulge and a portion of the disc in $Y Z J H K_{S}$ filters down to $K_{S} \sim 20$, and thus presents an ideal opportunity to study the GGCs located in the survey area. Since the advent of near-IR arrays, a wealth of effort has been devoted to studying GGCs in the near-IR largely by Valenti, Ferraro and collaborators (e.g. Ferraro et al. 2000; Valenti, Ferraro \& Origlia 2004a,b; Valenti, Ferraro \& Origlia 2010, hereafter V10; also see Chun et al. 2010, and references therein), in addition to the earlier studies of Cho \& Lee (2002) and Ivanov \& Borissova (2002) that employed photometry from the Two Micron All-Sky Survey (2MASS; Skrutskie et al. 2006). An important goal of these investigations was the construction of relations between observable features in 
cluster near-IR colour-magnitude diagrams (CMDs) and their chemical abundances, as these relations can then be applied to obtain photometric metallicity estimates. With an eye towards future application for distant and/or heavily extincted stellar systems, we revisit these calibrations. This is advantageous in light of not only the quality of the VVV photometry, but more importantly its widefield nature, facilitating a statistical assessment of contamination by field stars (see Section 2.5), leveraged together with improved spectroscopic abundances (see Section 4.1) and reddening maps (e.g. Alonso Garcia et al. 2012; Gonzalez et al. 2012; Cohen et al. 2014, see Section 2.4). Here, we analyse an initial subset of GGCs within the VVV survey area that have spectroscopically measured $[\mathrm{Fe} / \mathrm{H}]$ values, with the goal of constructing updated distance- and reddening-independent relations between photometric features observable on the cluster giant and horizontal branches (HBs) and their metallicities. The resulting relations between distance- and reddening-independent photometric features measured from nearIR cluster CMDs versus cluster metallicities are further optimized by concatenating the results presented here with those available in the literature.

In the next section, we present the details of our observations and data processing, including corrections for differential reddening and field star contamination, and the resulting cluster CMDs. In Section 3 , we describe our methodology for measuring cluster photometric features as well as their uncertainties, and in Section 4, we use these measurements, along with literature values, to construct relations that can be used to estimate metallicities of old stellar populations photometrically. In the final section, we summarize our results, discussing implications for clusters with controversial metallicity values.

\section{DATA PROCESSING}

\subsection{Target cluster selection}

There are 36 GGCs presently known in the area covered by the VVV survey according to the catalogue of Harris (1996, 2010 revision, hereafter $\mathrm{H} 10$ ), plus one candidate discovered as a result of this survey (VVV CL001; Minniti et al. 2011). We aim to derive relations between observed photometric parameters on the cluster red giant branches (RGBs), where the most IR-bright cluster members lie, and cluster metallicities (in terms of both $[\mathrm{Fe} / \mathrm{H}]$ and $[\mathrm{M} / \mathrm{H}]$ ), so we have selected a subset of the GGCs in the VVV survey area that all have spectroscopically measured $[\mathrm{Fe} / \mathrm{H}]$ values. To restrict our sample to only those clusters with high-quality $[\mathrm{Fe} / \mathrm{H}]$ measurements, we consider only clusters with a value of ' 1 ' in the last column of table A1 in Carretta et al. (2009, hereafter C09), and add two clusters (NGC 6380 and M 28 = NGC 6626) with recent spectroscopic $[\mathrm{Fe} / \mathrm{H}]$ values based on $\mathrm{Ca}$ II triplet equivalent widths (Saviane et al. 2012; Mauro et al. 2014, hereafter M14), comprising a sample of 17 GGCs from VVV including photometry of NGC 6544 described in Cohen et al. (2014). We return to the issue of various spectroscopic metallicities for the target clusters in Section 4.1, and the use of literature measurements for additional clusters is discussed in Section 4.3.

\subsection{Photometry}

The images that we employ were obtained as part of the VVV survey using the $4.1 \mathrm{~m}$ Visible and Infrared Survey Telescope for Astronomy (VISTA), equipped with the VIRCAM (VISTA InfraRed Camera) instrument (Emerson, McPherson \& Sutherland 2006).
The VIRCAM detector consists of a $4 \times 4$ array of chips, each with $2048 \times 2048$ pixels and a pixel scale of 0.339 arcsec per pixel. A description of the survey can be found in Minniti et al. (2011), with further details regarding the survey strategy and data products in Saito et al. (2010). Information regarding the first data release, including products that we employ here, is given in Saito et al. (2012). Point spread function-fitting (PSF) photometry is performed on VVV images obtained from the Cambridge Astronomical Survey Unit (CASU) ${ }^{1}$ via the iterative usage of the DAOPHOT/ALLFRAME suite (Stetson 1987, 1994) identically to previous studies (e.g. Mauro et al. 2012; Cohen et al. 2014). This PSF photometry pipeline has been customized to operate on pre-processed, stacked VVV images produced by CASU, and the reader is referred to Mauro et al. (2013) for a detailed description of the PSF photometry pipeline and comparisons with other data reduction techniques and products. We have chosen to perform photometric and astrometric calibration of the resulting catalogues to 2MASS for two reasons. First, because our photometry becomes saturated below the tip of the RGBs of all of our target clusters, merging our photometric catalogues with 2MASS is necessary in order to construct fiducial sequences and luminosity functions (LFs) over the entire luminosity range of the cluster RGBs and measure photometric features (described in Section 3). Secondly, by performing our analysis in the 2MASS photometric system, our results may be directly compared and/or combined with previous near-IR studies, the majority of which have been calibrated to 2MASS as well (Valenti et al. 2004a; V10; Chun et al. 2010; V10; Cohen et al. 2014, 2015). To calibrate our photometry and astrometry to the $2 \mathrm{MASS} J H K_{S}$ system, a magnitude range is selected among the stars matched between VVV and the 2MASS point source catalogue (PSC) in which both data sets show good agreement with minimal scatter, avoiding stars that are sufficiently faint so as to be unduly affected by crowding and/or large photometric errors in 2MASS. Additionally, stars with neighbours detected within 2.2 arcsec contributing a contaminating flux of $\geq 0.03$ mag are rejected from use as local standards (e.g. Mauro et al. 2013). Instrumental magnitudes resulting from PSF photometry are calibrated to the 2MASS $J H K_{S}$ system (rather than the native VISTA filter system) using the classical transformation equations of the form $m_{2 \mathrm{MASS}}-m_{\text {inst }}=a+b\left(J-K_{S}\right)_{2 \mathrm{MASS}}$, where $a$ is a photometric zero-point offset and $b$ is a linear colour term. The coefficients $a$ and $b$ are obtained independently for each VIRCAM chip per image per filter using least squares fitting, but using a weighting scheme to downweight discrepant data points ${ }^{2}$ rather than a sigma clipping or rejection procedure. For the coefficients $a$ and $b$, the values measured in each of the three $\left(J, H, K_{S}\right)$ filters are $a=$ $(0.62,0.26,-0.52) \pm(0.04,0.03,0.06)$ and $b=(0.03,-0.02,-0.02)$ $\pm(0.02,0.02,0.02)$, compared to median fitting uncertainties $\leq 0.02$ for the offset $a$ and $\leq 0.01$ for the colour term $b$ in all three bandpasses. Thus, the resulting photometric calibrations have $1 \sigma$ zeropoint uncertainties of $\lesssim 0.02$ mag for all target clusters, and a star by star comparison between our calibrated photometry and 2MASS in all three $J H K_{S}$ filters is shown in Fig. 1. All stars matched between VVV and 2MASS are shown in grey in each panel of Fig. 1, and

\footnotetext{
${ }^{1}$ Images and aperture photometry catalogues from VVV data releases are publicly available through the ESO archive, and CASU is located at http://casu.ast.cam.ac.ck

${ }^{2}$ The algorithm is based on a series of lectures presented at ' $\mathrm{V}$ Escola Avancada de Astrofisica' by P. B. Stetson, see http://ned.ipac.caltech.edu/ level5/Stetson/Stetson_contents.html and http://www.cadc.hia.nrc.gc.ca/ community/STETSON/homogenous/
} 


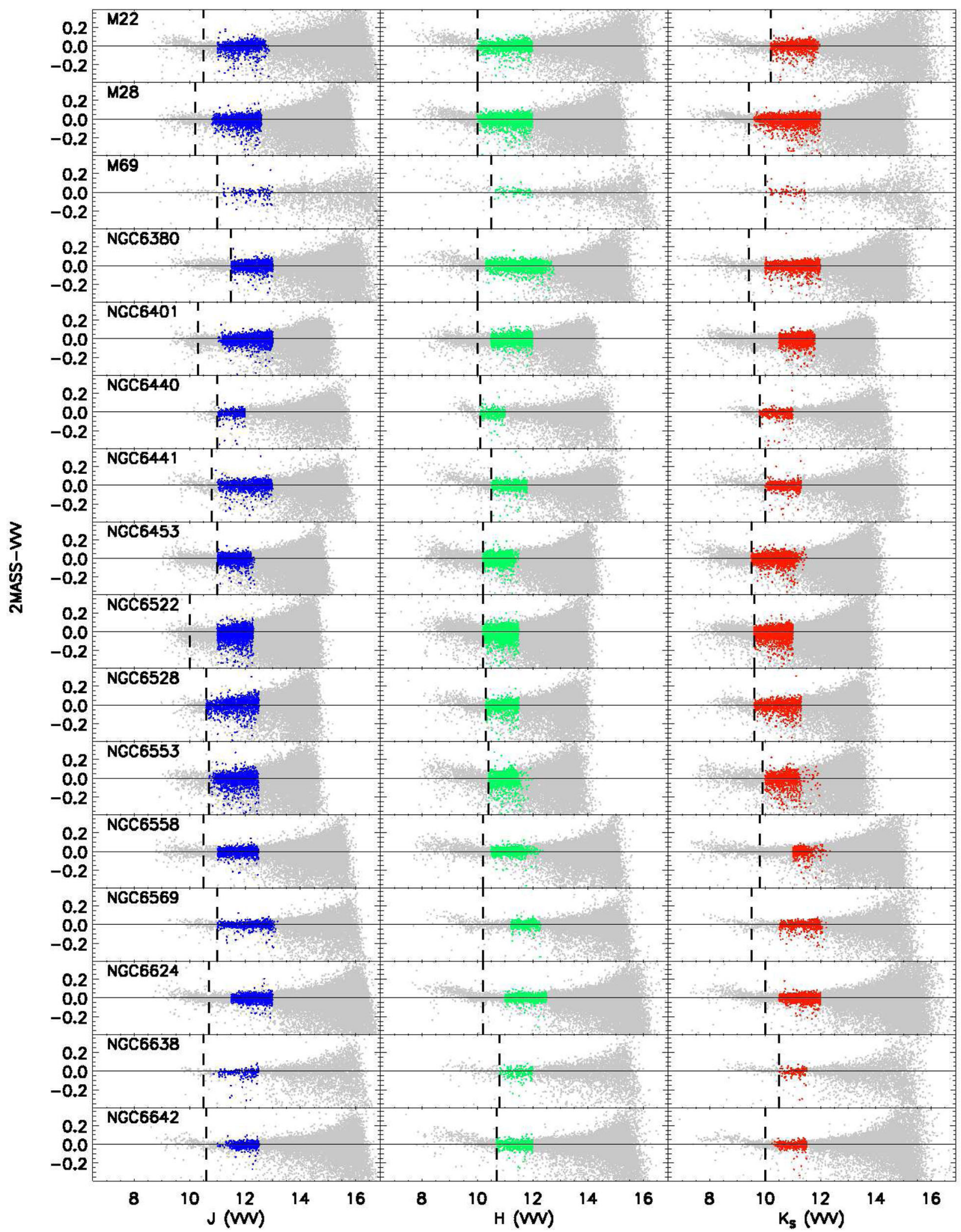

Figure 1. A comparison between our calibrated magnitudes and those from 2MASS for all of our target clusters. Each cluster is shown as a row of three plots, illustrating the difference between VVV and 2MASS as a function of (left to right) VVV $J, H$ and $K_{S}$ magnitude. In each plot, the grey points represent all stars matched between VVV and 2MASS, while the coloured points represent the stars used for calibration. The solid horizontal line represents equality, while the dashed vertical line indicates the VVV saturation limit above which photometry from 2MASS was employed. 
the subset of these stars used for calibration is overplotted. The vertical dashed line in each panel of Fig. 1 indicates the magnitude at which the VVV photometry is unusable due to saturation, that varies somewhat from cluster to cluster due to differences in stellar crowding as well as observing conditions. For stars that are brighter than this limit in any of the three $J H K_{S}$ filters, we supplement our VVV catalogues with photometry from the 2MASS PSC. All colours and magnitudes that we report in this study are in the 2MASS photometric system (rather than the native VISTA system), and additional discussions regarding the calibration of VVV photometry to the 2MASS system can be found in Moni Bidin et al. (2011) and Chené et al. (2012).

Astrometric calibration is performed to the coordinates given in the 2MASS PSC, using the world coordinate system information placed in the headers of the stacked VVV images by CASU as an initial guess in order to correct for effects of geometric distortion. The resulting astrometry has a root mean square (rms) precision of $\sim 0.2$ arcsec for all target clusters, in accord with the astrometric precision of 2MASS.

\subsection{Comparison with previous photometry}

Of our target clusters, 13 of 16 are also included in the compilation of V10. ${ }^{3}$ We calculate the mean magnitude differences in each filter between our photometry and theirs using a weighted $2.5 \sigma$ clip in magnitude bins, employing only unsaturated stars brightward of the observed LF peak. The resulting comparisons of magnitude difference as a function of magnitude are shown for each cluster in Fig. 2. Given our photometric zero-point uncertainty of $<0.02 \mathrm{mag}$ and the zero-point uncertainty of 0.05 mag estimated by V10, the two studies, having both been calibrated to 2MASS, are generally in good agreement. While larger offsets are seen in a few cases (NGC 6528, NGC 6553, NGC 6638, NGC 6642), the direct comparison with 2 MASS in Fig. 1 gives no reason to be doubtful about the calibration of these clusters. Specifically, the mean magnitude offset between the VVV and 2MASS photometry (weighted by the inverse square of their total photometric uncertainties) over the magnitude range of stars used for calibration is $<0.016 \mathrm{mag}$ in $J$ and $K_{S}$ and $<0.023 \mathrm{mag}$ in $H$ for these four clusters (these mean differences are $<0.02$ mag for all other clusters in all bandpasses as well).

Photometric analysis of GGCs towards the Galactic bulge can be severely hampered by contamination from field stars in the bulge and disc, particularly in cases where bulge and disc contaminants are inseparable from the cluster evolutionary sequences using colour-magnitude criteria alone. Statistical field star decontamination methods that compare the colour-magnitude loci of cluster and field stars generally rely on the assumption that reddening is spatially invariant (see Section 2.5.1 below), so before undertaking analyses of the GGC photometry, we first correct for differential reddening and then apply a statistical field star decontamination procedure.

\subsection{Differential reddening}

We correct our photometric catalogue of each cluster for reddening only in a strictly differential sense (we do not correct for total line-of-sight extinction). This is done using the reddening maps of

\footnotetext{
${ }^{3}$ See the Bulge Globular Cluster Archive at http://www.bo.astro.it/ GC/ ir_archive
}

Gonzalez et al. (2012), ${ }^{4}$ adopting the value of $E\left(J-K_{S}\right)$ corresponding to the location of the cluster centre as a reference zero-point for the differential reddening corrections over the spatial area of each cluster. This reference value is given as $E\left(J-K_{S}\right)_{\mathrm{REF}}$ in Table 1 . The photometric catalogue for each cluster is then corrected for reddening variations over the field of view using the difference between the value of $E\left(J-K_{S}\right)$ at a given spatial location and $E\left(J-K_{S}\right)_{\text {REF }}$ (i.e. the value at the spatial location of the cluster centre). However, since the Gonzalez et al. (2012) maps were constructed by measuring the variation in the $\left(J-K_{S}\right)$ colour of the Galactic bulge red clump (RC) as a function of spatial location, the number statistics necessary to reliably measure the bulge RC colour restrict the spatial resolution of the Gonzalez et al. (2012) maps to $>1$ arcmin, while significant differential reddening towards bulge GGCs can occur on spatial scales of arcseconds (Alonso Garcia et al. 2012; Massari et al. 2012; Cohen et al. 2014). Furthermore, the Gonzalez et al. (2012) maps were constructed from aperture photometry catalogues rather than PSF photometry, and therefore suffer from crowding and incompleteness significantly brightward of their detection limits as compared to PSF photometry (e.g. Mauro et al. 2013, see their fig. 6). Therefore, where available, we have combined the Gonzalez et al. (2012) maps of the field surrounding each cluster with high-spatial resolution reddening maps (constructed using cluster stars) of the central region of the cluster. The high-resolution maps were taken from Alonso Garcia et al. (2012) where available (eight clusters), from Cohen et al. (2014) in the case of NGC 6544, and for six more clusters, we employ maps similarly constructed from archival optical Hubble Space Telescope imaging described in detail elsewhere (Cohen et al., in preparation). ${ }^{5}$ While the high-resolution maps are generally restricted to the inner regions of the target clusters where the membership probability is high, we note that they extend well beyond the cluster half-light radii from the Harris (1996) and H10 catalogue, encompassing the majority of cluster members. ${ }^{6}$ These high-resolution maps are also applied in a strictly differential sense, relative to $E\left(J-K_{S}\right)_{\mathrm{REF}}$, but we must take into account that the differential reddening corrections given by the Alonso Garcia et al. (2012) maps may not be referred to the same differential reddening zero-point (i.e. the cluster centre). Therefore, we shift the Alonso Garcia et al. (2012) corrections to refer to our reference value of $E\left(J-K_{S}\right)_{\mathrm{REF}}$ (i.e. the Gonzalez et al. 2012 value at the cluster centre) by comparing, for all stars within the radius permitted by the Alonso Garcia et al. (2012) maps, the $\left(J-K_{s}\right)$ colour obtained after performing the Alonso Garcia et al. (2012) correction with that resulting from the Gonzalez et al. (2012) correction. This yields the mean difference $\Delta E\left(J-K_{S}\right)$ (and standard deviation) between the two maps, given in Table 1 for clusters in our sample with high-resolution maps from Alonso Garcia et al. (2012). For the two target clusters with no available high-spatial resolution reddening maps (NGC 6569 and NGC 6638), we employ only the Gonzalez et al. (2012) maps, noting that they predict quite modest differential reddening over the entire sampled area in both cases $\left(\Delta E\left(J-K_{S}\right) \leq 0.065\right)$.

\footnotetext{
${ }^{4}$ The BEAM calculator can be found at http://mill.astro.puc.cl/BEAM/ calculator.php

${ }^{5}$ For comparison, we note that these maps have a median spatial resolution of $\sim 10$ arcsec.

${ }^{6}$ The only possible exception is NGC6558, for which the Alonso Garcia et al. (2012) map has a radial limit of 1.81 arcmin versus a half-light radius of 2.15 arcmin from H10, although this value may not be too reliable as this cluster is core-collapsed (Trager, King \& Djorgovski 1995).
} 


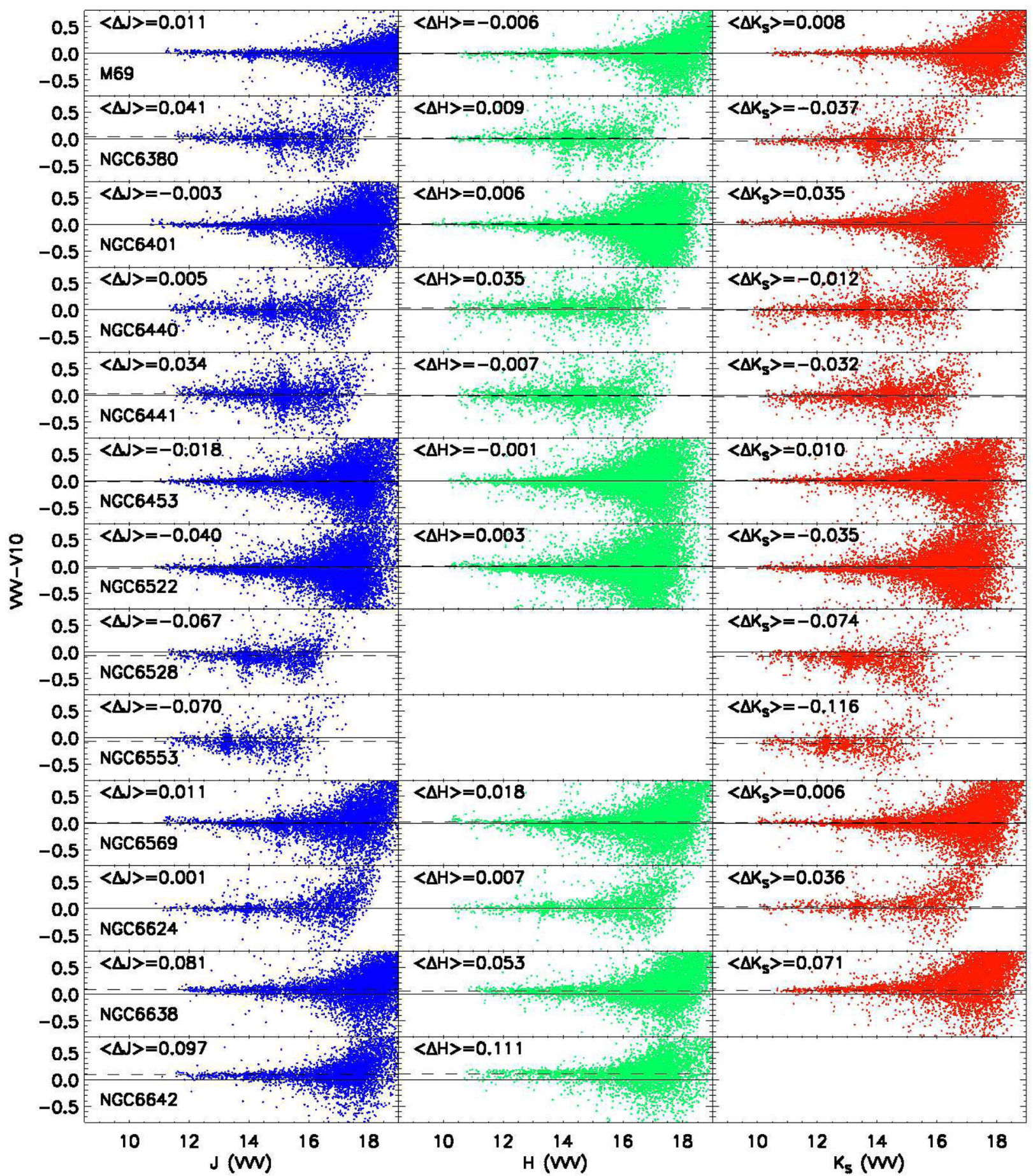

Figure 2. A comparison between our photometry and that of V10. Symbols are as in Fig. 1 except that the mean magnitude offset is given in each plot and shown as a horizontal dashed line.

\subsection{Field star decontamination}

\subsubsection{Methodology}

We clean our differential reddening corrected cluster CMDs of field stars using a statistical technique detailed in Bonatto \& Bica (2007), including recent improvements described by Bonatto \& Bica (2010). The application of this technique to VVV PSF photometry is described in Cohen et al. (2014), but can be summarized as follows: two spatial regions are selected, the first being the spa- tial region to be decontaminated (over which high-spatial resolution differential reddening maps are available) that has area $A_{\text {clus }}$ and a total number of stars $N_{\text {tot }}$ in the magnitude range considered for decontamination. The second area is the comparison (e.g. field) region, that has area $A_{\mathrm{fld}}$, which we have chosen to have an inner radius equal to the cluster $\mathrm{H} 10$ tidal radii. ${ }^{7}$ To statistically decontaminate

${ }^{7}$ For NGC 6569 and NGC 6638, that lack high-resolution differential reddening maps, we set the cluster area to have limiting radii of $r \leq 1.90$ and 
Table 1. Differential reddening and decontamination parameters.

\begin{tabular}{|c|c|c|c|c|c|c|c|c|c|}
\hline Cluster & $\begin{array}{c}E\left(J-K_{S}\right)_{\mathrm{REF}} \\
(\mathrm{mag})\end{array}$ & $\begin{array}{c}\left\langle\Delta E\left(J-K_{S}\right)\right\rangle^{a} \\
(\mathrm{mag})\end{array}$ & Reddening map ${ }^{b}$ & $N_{\text {tot }}$ & $N_{\text {clus }}$ & $\mathrm{A}_{\text {fld }} / \mathrm{A}_{\text {clus }}$ & $\begin{array}{c}A_{\mathrm{fld}} \\
\left(\operatorname{arcmin}^{2}\right)\end{array}$ & $\begin{array}{c}f_{\text {sub }} \\
\text { ( per cent) }\end{array}$ & $\begin{array}{c}J_{\lim } \\
(\mathrm{mag})\end{array}$ \\
\hline NGC6380 & 0.496 & & 1 & $5169 \pm 72$ & $4292 \pm 148$ & 117.67 & 854.10 & $99.8 \pm 0.3$ & 18.40 \\
\hline NGC6401 & 0.417 & & 1 & $11385 \pm 107$ & $6086 \pm 403$ & 61.95 & 732.46 & $99.3 \pm 0.4$ & 18.40 \\
\hline NGC6440 & 0.530 & & 1 & $6413 \pm 80$ & $4443 \pm 164$ & 49.77 & 366.00 & $99.8 \pm 0.1$ & 18.40 \\
\hline NGC6441 & 0.205 & & 1 & $15050 \pm 123$ & $10860 \pm 120$ & 39.07 & 495.92 & $99.9 \pm 0.1$ & 18.80 \\
\hline NGC6453 & 0.285 & & 1 & $6616 \pm 81$ & $4717 \pm 46$ & 149.67 & 1100.67 & $99.9 \pm 0.1$ & 18.20 \\
\hline NGC6522 & 0.234 & $-0.018 \pm 0.017$ & 2 & $21244 \pm 146$ & $14942 \pm 591$ & 75.13 & 1281.29 & $99.9 \pm 0.1$ & 18.40 \\
\hline NGC6528 & 0.271 & & 1 & $11857 \pm 109$ & $4231 \pm 209$ & 32.74 & 367.41 & $99.7 \pm 0.1$ & 18.40 \\
\hline NGC6544 & 0.736 & & 3 & $24166 \pm 155$ & $7771 \pm 235$ & 32.80 & 368.57 & $93.7 \pm 1.2$ & 18.84 \\
\hline NGC6553 & 0.369 & $0.008 \pm 0.029$ & 2 & $48836 \pm 221$ & $29283 \pm 412$ & 11.63 & 558.43 & $99.8 \pm 0.1$ & 18.20 \\
\hline NGC6558 & 0.150 & $0.004 \pm 0.016$ & 2 & $6678 \pm 82$ & $3707 \pm 87$ & 87.20 & 897.46 & $99.5 \pm 0.2$ & 18.60 \\
\hline NGC6569 & 0.199 & & 4 & $7692 \pm 88$ & $5744 \pm 33$ & 32.35 & 366.92 & $99.9 \pm 0.1$ & 18.60 \\
\hline NGC6624 & 0.104 & $0.003 \pm 0.021$ & 2 & $24038 \pm 155$ & $14537 \pm 574$ & 26.57 & 1282.67 & $99.9 \pm 0.1$ & 19.20 \\
\hline M28 & 0.138 & $0.014 \pm 0.027$ & 2 & $56829 \pm 238$ & $33320 \pm 88$ & 16.56 & 1274.84 & $99.9 \pm 0.1$ & 18.60 \\
\hline M69 & 0.017 & $0.004 \pm 0.009$ & 2 & $14107 \pm 119$ & $5079 \pm 389$ & 0.53 & 17.61 & $98.1 \pm 0.6$ & 18.68 \\
\hline NGC6638 & 0.189 & & 4 & $9654 \pm 98$ & $3466 \pm 358$ & 4.66 & 35.19 & $99.1 \pm 0.2$ & 18.93 \\
\hline NGC6642 & 0.161 & $0.002 \pm 0.027$ & 2 & $9853 \pm 99$ & $6626 \pm 76$ & 39.79 & 616.03 & $99.9 \pm 0.1$ & 19.40 \\
\hline M22 & 0.000 & $0.042 \pm 0.044$ & 2 & $153216 \pm 391$ & $65122 \pm 4052$ & 1.69 & 224.28 & $96.5 \pm 0.8$ & 18.45 \\
\hline
\end{tabular}

${ }^{a}$ Reddening map zero-point offset in the sense (Alonso Garcia et al. 2012)-(Gonzalez et al. 2012)

${ }^{b}$ Reddening maps applied to cluster photometry before decontamination as follows: (1) Cohen et al. (in preparation) (2) Alonso Garcia et al. 2012,

(3) Cohen et al. 2014, (4) Gonzalez et al. 2012

the cluster region, the CMD of the cluster region is compared to the CMD of the comparison region by dividing their CMDs into a three-dimensional grid of cells in $J,\left(J-K_{S}\right),(J-H)$. The effects of photometric incompleteness are minimized by including only stars that lie brightward of the observed cluster area LF peak $J_{\text {lim }}$. In each CMD cell of the cluster region, the number of field stars to be removed is calculated by summing the probability density distributions of all comparison field stars in the analogous CMD cell, corrected for the ratio of cluster to comparison field areas. This number of stars, rounded to the nearest integer, are randomly removed from the cluster region CMD cell, and the entire procedure is repeated over $3^{6}=729$ iterations in which the cell sizes and locations are varied to mitigate the effects of binning. The mean number of surviving cluster stars $N_{\text {clus }}$ is calculated over all iterations, stars are sorted by their survival frequency and cluster stars are retained in order of decreasing survival frequency until this mean number of surviving cluster stars is reached. The efficiency of this field star decontamination procedure may be gauged using the subtraction efficiency $f_{\text {sub }}$, that is the fraction of (decimal) stars to be subtracted (based on the stellar density of the comparison field and the ratio of comparison to cluster field area) to the actual (integer) number of probable field stars removed from the cluster region. To attain the highest possible subtraction efficiencies, the comparison regions generally consist of an annulus wide enough that $A_{\text {fld }}$ is many times larger than $A_{\text {clus }}$. A large comparison region has the added advantage that any small-scale variations in the stellar density of the comparison field are averaged out, as the comparison regions we employ have typical areas $\gtrsim 10^{3} \operatorname{arcmin}^{2}$. However, especially given the relatively large ( $\sim 30$ arcmin) tidal radii of some of our target clusters, in practice, an upper limit to the size of the comparison region is necessary due to several factors. These include the proximity of other nearby features not representative of the cluster line of sight such as other globular and open clusters, and in the case of M69, proximity to the edge of the VVV survey area over which photom-

1.55 arcmin, respectively, from the cluster centre, corresponding to more than twice the $\mathrm{H} 10$ half-light radii in both cases. etry is available. The values of $N_{\text {tot }}, N_{\text {clus }}$, the ratio of comparison to cluster region areas $A_{\mathrm{fld}} / A_{\text {clus }}$, the total comparison region area $A_{\mathrm{fld}}$, the subtraction efficiency $f_{\text {sub }}$ and the faint magnitude limit $J_{\text {lim }}$ are given for all of our target clusters, including results for NGC 6544 from Cohen et al. (2014) that we add to our sample, in Table 1, along with formal uncertainties that take into account both photometric errors and Poissonian uncertainties of the total number of stars in the cluster and comparison regions. The impact of uncertainties in the decontamination procedure on the photometric features that we measure are discussed in the context of each of these features in Sections 3.2, 3.3.2 and 4.4.2.

\subsubsection{Proper motions: an independent test of the decontamination algorithm}

As an independent test of the decontamination procedure, we may compare our statistically decontaminated CMDs with results from relative proper motion studies. There is one cluster in our sample, M 22, for which membership probabilities have been calculated from relative proper motions over a relatively wide field of view by Libralato et al. (2014). ${ }^{8}$ After matching our photometric catalogue to theirs, in Fig. 3, we compare all stars in our (differential reddeningcorrected) catalogue surviving statistical decontamination (shown in panel a) with those that Libralato et al. (2014) considered likely members (panel b) as well as those that survived the decontamination procedure but have zero probability of membership according to their proper motions (panel c). It is evident that for this cluster, the decontamination procedure fails to remove a minority of field RGB stars, seen 0.2-0.3 mag redward of the cluster RGB in panels (a) and (c) of Fig. 3. There are several probable causes for this effect, and proper motion selection can be similarly subject to contamination from field stars with cluster-like proper motions (e.g. Libralato et al. 2015), although it may be possible to take this effect into account statistically in some cases (e.g. Milone et al. 2012).

\footnotetext{
${ }^{8}$ Proper motions in M22 were also published by Zloczewski et al. (2012), although they did not give formal membership probabilities.
} 


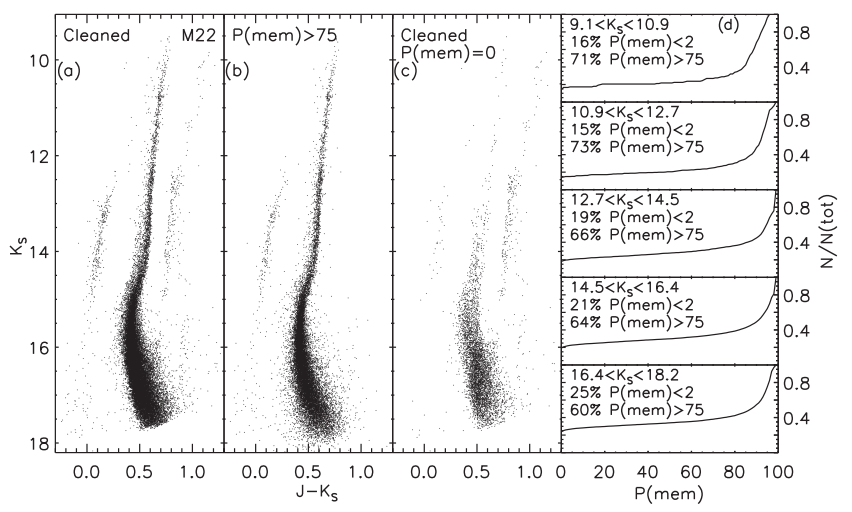

Figure 3. (a) CMD of all stars present in the proper motion catalogue of Libralato et al. (2014) that passed the statistical decontamination procedure described in Section 2.5.1. (b) All stars in our photometric catalogue that are likely proper motion members $\left(P_{\text {mem }}>75\right)$ according to Libralato et al. (2014). (c) Stars that survived our statistical decontamination algorithm but have a proper motion based membership probability of 0 from Libralato et al. (2014). (d) Cumulative distribution of Libralato et al. (2014) membership probability for all stars that survived the statistical decontamination procedure, shown in 5 mag bins.

To further compare the performance of the decontamination algorithm versus the use of proper motions as a function of magnitude (or, equivalently, photometric error), in panel (d) of Fig. 3, we divide the stars in our catalogue that survived the decontamination algorithm into magnitude bins. In each magnitude bin, we plot the cumulative distribution of the proper motion membership probabilities from Libralato et al. (2014), as well as giving the fraction of surviving stars in each bin that fall into the ranges of proper motion probability used by Libralato et al. (2014) to identify definite members $\left(P_{\text {mem }}>75\right.$ per cent $)$ and definite non-members $\left(P_{\text {mem }}<\right.$ 2 per cent). It is clear from the right-hand panel of Fig. 3 that the contamination rate among our statistically decontaminated sample is $\leq 25$ per cent without the use of a colour cut, and this contamination rate does not vary appreciably with magnitude.

\subsection{Colour-magnitude diagrams}

CMDs of all of our target clusters are shown in the $\left(K_{S}, J-K_{S}\right)$ plane in Fig. 4 and in the $(J, J-H)$ plane in Fig. 5, and are also included in the supplementary figures along with the RGB LFs. Stars that passed the decontamination procedure are shown in black, whereas stars that failed are shown in grey. In addition, we have identified known variables in our target clusters by matching our 2MASSastrometrized $J H K_{S}$ catalogues with the most recent version of the Catalogue of Variable Stars in GGCs (Clement et al. 2001) ${ }^{9}$ and the catalogue of equatorial coordinates by Samus et al. (2009). These variables are overplotted on the CMDs as blue diamonds. We have excluded known variables from the determination of the fiducial sequences since detailed variability studies show that asymptotic giant branch (AGB) variables may be present faintward of the RGB tip, and a more thorough discussion of variability on the upper RGB and the inclusion of variables in RGB tip magnitude measurements can be found in Section 3.2. In any case, the influence of known variables on each of the photometric features we measure is discussed in the context of each of the relevant features in Section 3.

\footnotetext{
${ }^{9}$ http://www.astro.utoronto.ca/ cclement/read.html
}

While in a minority of cases, the decontamination procedure results in gaps in the cluster evolutionary sequences or a failure to remove field RGB stars, this is a likely consequence of the differing spatial resolution between the reddening maps applied to the comparison field ( $>1$ arcmin; Gonzalez et al. 2012) and those applied to the cluster regions before decontamination (see Alonso Garcia et al. 2012; Cohen et al. 2014, Section 2.4). In addition, the fact that the clusters that are most susceptible to this effect (NGC 6553 and M22) are also the most nearby along the line of sight suggests that this could also be partially due to preferential obscuration of the field (e.g. bulge) population by the cluster in these cases, and we note that the Gonzalez et al. (2012) map that we employ for the comparison field tends to overestimate reddening at small $(<4 \mathrm{kpc})$ heliocentric distances (Schultheis et al. 2014). In any case, we find that the location of photometric features we measure is insensitive to this effect beyond their reported uncertainties, based both on comparisons to previous studies employing radial cuts and/or proper motions (see Section 3.5) as well as a comparison between values measured using statistically decontaminated CMDs versus field-subtracted LFs (see Section 3.3.2).

\section{OBSERVED PHOTOMETRIC FEATURES}

\subsection{Cluster fiducial sequences}

In order to derive calibrations between cluster chemical abundances and photometric features along the cluster RGBs, including the RGB tip, bump and slope, we fit fiducial sequences to the RGBs in the differential reddening corrected, field star decomtaminated CMDs, that are hereafter referred to as the 'processed' CMDs. Fiducial sequences are fit using an iterative procedure similar to previous studies (Ferraro et al. 2000; Valenti et al. 2004a; Cohen et al. 2014, 2015). First, a rough visual colour-magnitude cut was used to isolate the CMD region of the RGB. Next, the RGB was divided into magnitude bins of width $0.5 \mathrm{mag}$, and the median colour and magnitude in each bin was measured. A low-order $(\leq 3)$ polynomial was then fit to these median colours as a function of magnitude, iteratively rejecting stars more than $2 \sigma$ in colour from the fit polynomial in each bin. This process is repeated until convergence is indicated by the number of surviving stars changing by under 2 per cent since the previous iteration. This procedure is still necessary even if the field star decontamination algorithm functions perfectly, since bona fide cluster HB and AGB stars should still be present and thus can be removed from consideration in a statistical manner to construct sequences representative of the RGB.

Once the fiducial sequence has been constructed, we make a colour cut in the $\left(K_{S}, J-K_{S}\right)$ CMD to identify sub-samples of stars used to measure the slope of the upper RGB as well as the locations of the red giant branch bump (RGBB) and the HB. Specifically, in order to minimize contamination of the cluster RGB by the $\mathrm{HB}$, we use only stars with $\left(J-K_{S}\right)$ colours within $3 \sigma$ of the fiducial sequence (where $\sigma$ represents the median photometric error as a function of $K_{S}$ magnitude), that we refer to as Sample A. Similarly, to avoid a bias on the HB magnitude caused by the RGBB, we measure the location of the HB using only stars blueward of Sample A, that we refer to as Sample B. An example of the selection of both of these samples is shown for the case of M69 in Fig. 6.

The coefficients of the fiducial sequences in both the $\left(K_{S}, J-K_{S}\right)$ and $(J, J-H)$ CMDs are given in Table 2 along with their ranges of 


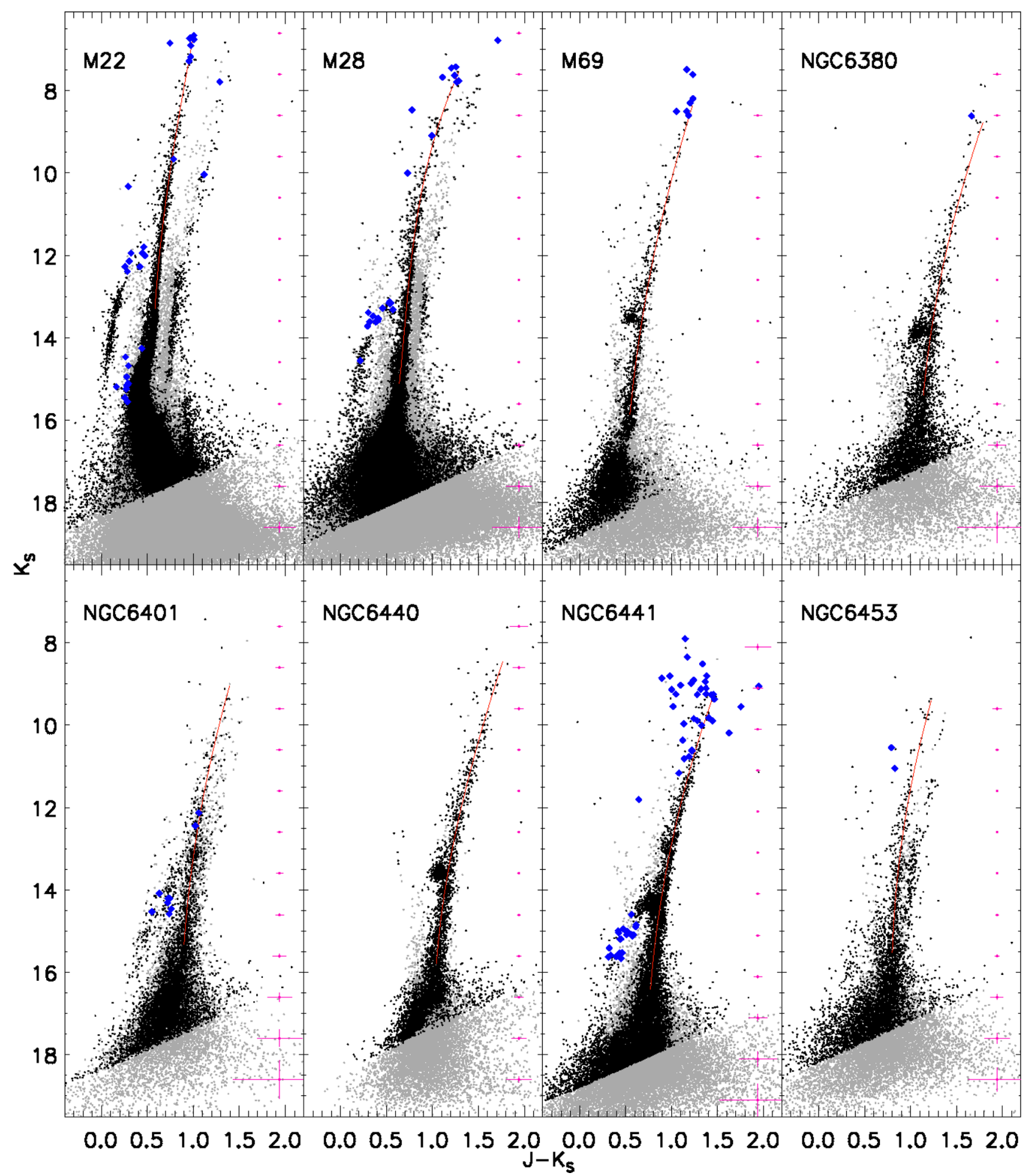

Figure 4. Differential reddening corrected CMDs of all of our target clusters in the $\left(K_{S}, J-K_{S}\right)$ plane. All stars within the cluster region are shown in grey, those surviving the decontamination procedure are shown in black, and known variables that were removed from our analysis are plotted as blue diamonds. In addition, our fiducial sequences are shown in red, and median photometric errors in magnitude bins are shown along the right-hand side of each CMD in magenta. High-quality $\left(K_{S}, J-K_{S}\right)$ and $(J, J-H)$ CMDs for all target clusters are included in as a set of supplementary figures.

validity. We reiterate that these fiducial sequences are derived from photometry that has been corrected for differential reddening across the cluster relative to the cluster centre, but has not been corrected for total line-of-sight extinction.

\subsection{Red giant branch tip}

For the clusters in common with V10, we could, in principle, use their measured red giant branch tip (TRGB) magnitudes, since both 


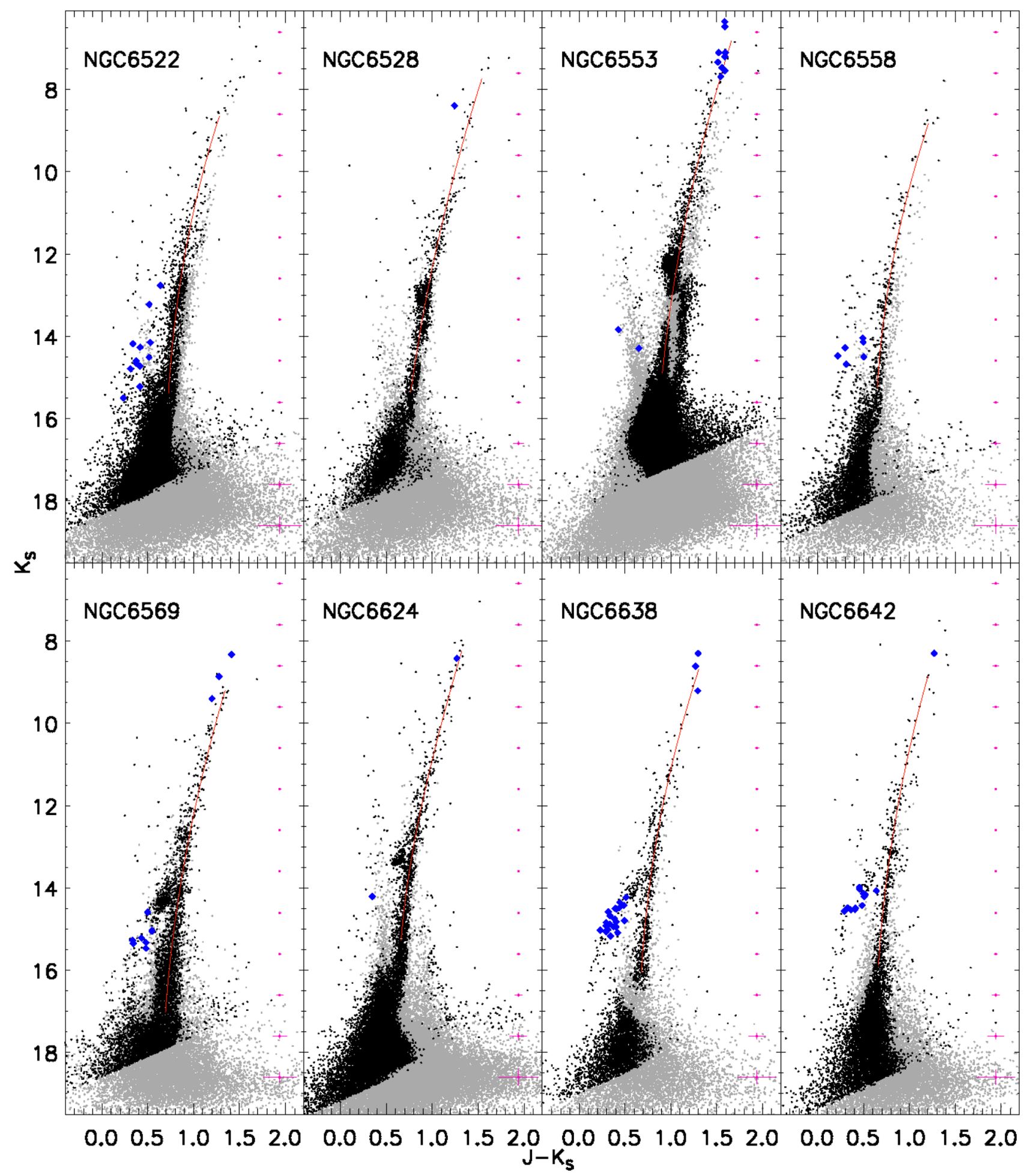

Figure 4 - continued

studies are similarly reliant upon 2MASS photometry at these bright magnitudes, and they likewise applied a statistical procedure to remove field stars from 2MASS photometry. However, we redetermine these magnitudes for three reasons. First, application of differential reddening corrections could change these values somewhat (although this effect would likely be small due to the horizontality of the reddening vector in $\left.K_{S},\left(J-K_{S}\right)\right)$. Secondly, we ensure that all clusters (not just those in common with V10) have their TRGB magnitudes measured self-consistently. Thirdly, we avoid luminous AGB variables unknown in previous investigations (but see below). Therefore, when identifying the location of the TRGB, we use the processed CMDs as they take the photometry in all three $J H K_{S}$ bands as well as photometric errors into consideration, and select the brightest star along the cluster RGB in both the $K_{S},\left(J-K_{S}\right)$ and $J,(J-H)$ decontaminated CMDs. In addition, we have checked the candidate TRGB star in each cluster against unmatched stars from 


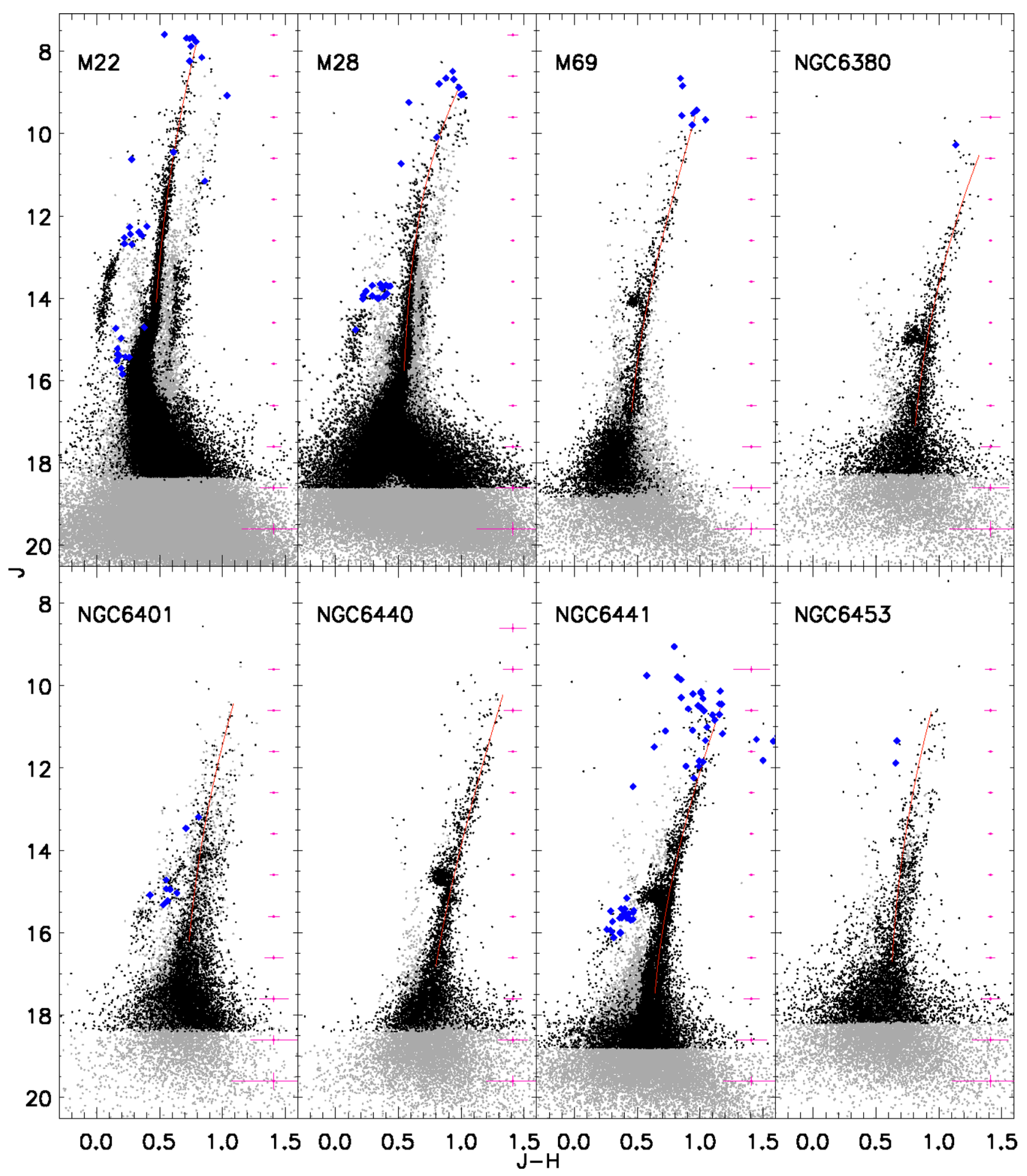

Figure 5. As for Fig. 4 but in the $(J, J-H)$ plane.

2MASS within the H10 tidal radii as well as unmatched stars in the catalogues of V10 where available.

In many cases, selection of the brightest non-AGB cluster member is ambiguous, so the selected TRGB star is ultimately only the candidate brightest RGB star. Although a statistical uncertainty on the TRGB magnitude can be estimated based on evolutionary considerations (e.g. Ferraro et al. 2000), the true uncertainty in the TRGB location may be difficult to ascertain for three reasons.
First, the exponential nature of the RGB LF implies that the RGB is sparsely populated close to the tip. Even globular clusters typically have too few RGB stars to employ statistical methods for quantifying the TRGB uncertainty, such as the edge detection technique pioneered by Lee, Freedman \& Madore (1993) or maximum likelihood methods (Méndez et al. 2002; Makarov et al. 2006 but see Conn et al. 2011). Secondly, it is difficult to separate RGB and AGB members based on photometry since AGB stars are effec- 


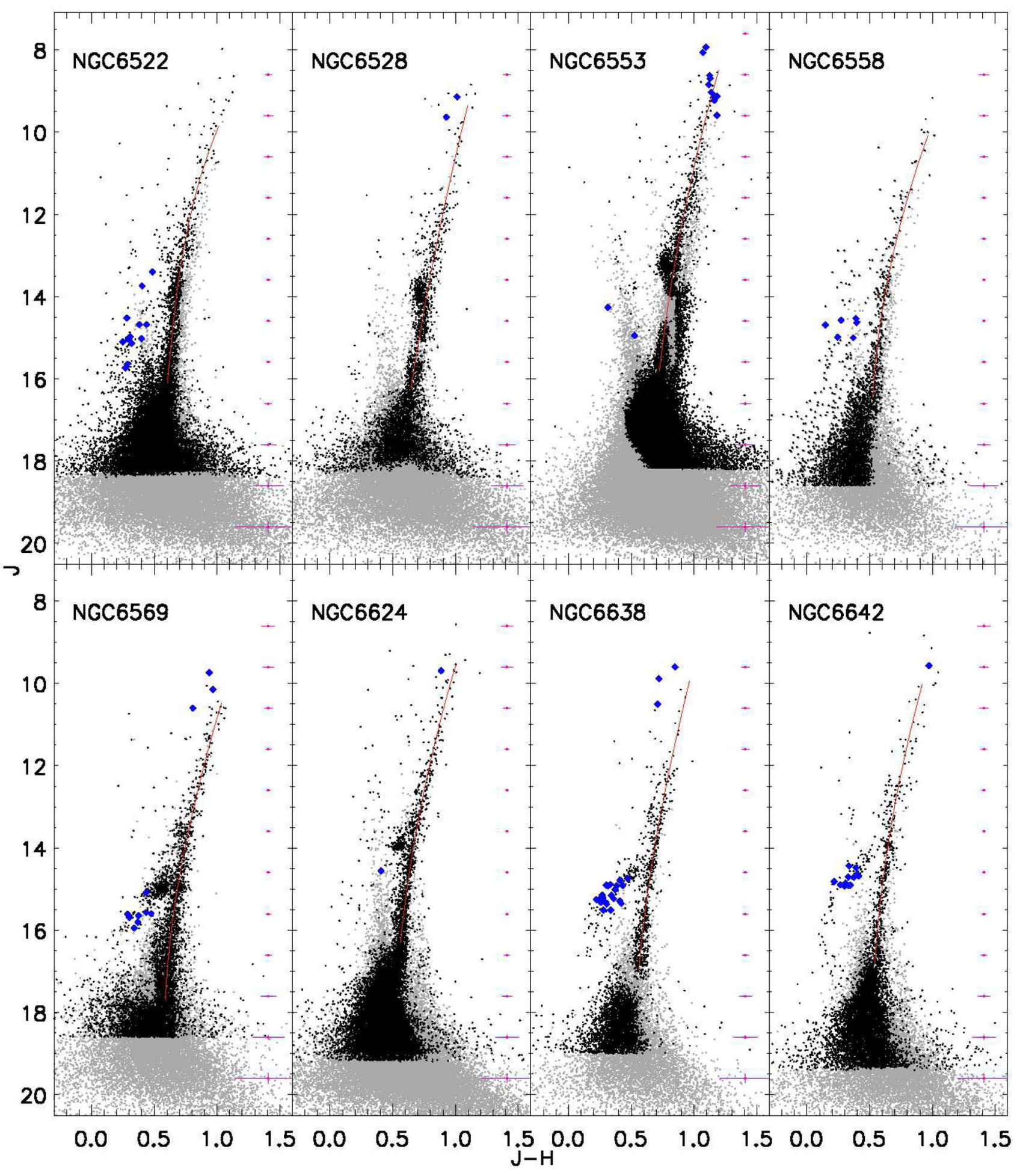

Figure 5 - continued

tively co-located with the TRGB in near-IR colour-magnitude and colour-colour planes. This is illustrated in a near-IR two-colour diagram in Fig. 7, where we plot AGB variables in NGC 362, NGC 2808 and M 22 from Lebzelter \& Wood (2011) and Sahay, Lebzelter $\&$ Wood (2014) as diamonds. We have included only periodic variables that those studies do not suspect of being non-members, and observed colours were converted to the dereddened plane using $E(B$ $-V$ ) values from VandenBerg et al. (2013; or Monaco et al. 2004 in the case of M22) and the $R_{V}=3.1$ extinction law from appendix B of Hendricks et al. (2012). To illustrate the coincidence of these variables with GGC RGBs, we overplot $\mathrm{K}$ and $\mathrm{M}$ giant colours from Bessell \& Brett (1988) as well as predictions of $12 \mathrm{Gyr} \alpha$ enhanced $([\alpha / \mathrm{Fe}]=+0.4$ for $[\mathrm{Fe} / \mathrm{H}]<0$, otherwise $[\alpha / \mathrm{Fe}]=$ $+0.2)$ isochrones over a wide range of cluster metallicities $(-2.5$ $<[\mathrm{Fe} / H]<+0.5)$ from the Dartmouth Stellar Evolution Database (DSED; Dotter et al. 2008) as these models reproduce GGC RGB 


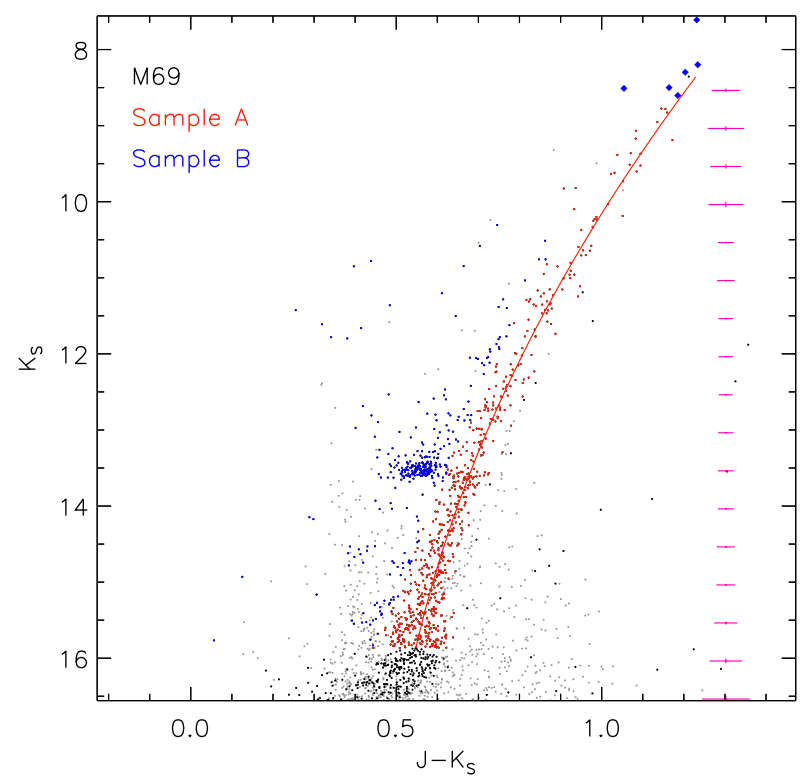

Figure 6. The processed CMD of M69, illustrating the selection of stellar sub-samples used to measure CMD features. Sample A, used to measure the RGB slope and the RGBB magnitude, is shown in red, and Sample B, used to measure the magnitude of the HB, is shown in blue. Other symbols are as in Fig. 4.
near-IR colours to $\sim 0.03 \mathrm{mag}$ (Cohen et al. 2015). Additionally, dereddened colours of Mira variables towards the Galactic bulge from the surveys of Matsunaga, Fukushi \& Nakada (2005) and Matsunaga et al. (2009) are shown as filled grey circles and crosses, respectively. Fig. 7 illustrates that variability is common close to the TRGB in the near-IR colour-colour plane as well as optical and near-IR CMDs. The problem of disentangling bright AGB and RGB cluster members is not restricted to more metal-rich GGCs, as periodic variables likely to be cluster members have also been detected in GGCs as metal-poor as M 15 (McDonald et al. 2010), that has $[\mathrm{Fe} / \mathrm{H}]=-2.33(\mathrm{C} 09)$, in addition to the metal-intermediate to metal-poor GGCs with variables shown in Fig. 7.

Thirdly, both AGB and RGB stars are often photometrically variable, so that even when an AGB star has a colour and/or magnitude that is separable from the RGB in the mean, it may coincide with the RGB at some pulsational phases. This is illustrated, for example, in fig. 6 of Montegriffo et al. (1995), where the location of AGB variables near the RGB tip on the CMD changes significantly as a function of their pulsational phase. However, not only do AGB and RGB stars both vary, but it is unclear whether their pulsational properties can be used to disentangle their evolutionary state. For example, the optical variability study of 47 Tuc by Lebzelter \& Wood (2005) found that all cluster giants that they detected with $(V-I)>1.8$ are variable (see their fig. 3 ). Furthermore, while upper

Table 2. Coefficients of observed fiducial sequences.

\begin{tabular}{|c|c|c|c|c|c|c|}
\hline \multicolumn{7}{|c|}{$\left(J-K_{S}\right)=a_{0}+a_{1} K_{S}+a_{2} K_{S}^{2}+a_{3} K_{S}^{3}$} \\
\hline Cluster & $K_{S, \min }$ & $K_{\mathrm{S}, \max }$ & $a_{0}$ & $a_{1}$ & $a_{2}$ & $a_{3}$ \\
\hline NGC6380 & 8.770 & 15.609 & 3.7852440 & -0.3019084 & 0.0084423 & $0.000 \mathrm{v} 0000$ \\
\hline NGC6401 & 9.040 & 15.728 & 3.1537068 & -0.2626610 & 0.0075405 & 0.0000000 \\
\hline NGC6440 & 8.459 & 15.986 & 3.0384719 & -0.1304930 & -0.0054349 & 0.0003610 \\
\hline NGC6441 & 9.188 & 16.612 & 3.6435592 & -0.3149566 & 0.0085259 & 0.0000000 \\
\hline NGC6453 & 9.402 & 15.720 & 3.1904884 & -0.2939827 & 0.0090236 & 0.0000000 \\
\hline NGC6522 & 8.649 & 15.589 & 3.4198779 & -0.3620996 & 0.0147217 & -0.0001667 \\
\hline NGC6528 & 7.739 & 15.560 & 2.9406701 & -0.2207616 & 0.0051508 & 0.0000000 \\
\hline NGC6553 & 6.812 & 15.106 & 2.8970294 & -0.2216789 & 0.0059164 & 0.0000000 \\
\hline NGC6558 & 8.822 & 15.551 & 3.2531687 & -0.3158048 & 0.0095168 & 0.0000000 \\
\hline NGC6569 & 9.192 & 17.227 & 3.0955093 & -0.2485383 & 0.0063109 & 0.0000000 \\
\hline NGC6624 & 8.234 & 15.478 & 2.2191760 & -0.0562974 & -0.0103873 & 0.0004842 \\
\hline M28 & 7.548 & 15.314 & 4.9231014 & -0.8379519 & 0.0577563 & -0.0013936 \\
\hline M69 & 8.358 & 16.068 & 2.8374305 & -0.2459917 & 0.0064082 & 0.0000000 \\
\hline NGC6638 & 8.682 & 16.256 & 3.1853596 & -0.2878985 & 0.0082148 & 0.0000000 \\
\hline NGC6642 & 8.830 & 17.089 & 2.8574505 & -0.2486005 & 0.0069500 & 0.0000000 \\
\hline M22 & 6.722 & 13.500 & 1.5011816 & -0.0506029 & -0.0058926 & 0.0003390 \\
\hline \multicolumn{7}{|c|}{$(J-H)=a_{0}+a_{1} J+a_{2} J^{2}+a_{3} J^{3}$} \\
\hline Cluster & $J_{\min }$ & $J_{\max }$ & $a_{0}$ & $a_{1}$ & $a_{2}$ & $a_{3}$ \\
\hline NGC6380 & 10.526 & 17.123 & 3.4250948 & -0.2755087 & 0.0071731 & 0.0000000 \\
\hline NGC6401 & 10.436 & 16.290 & 2.6685162 & -0.2095136 & 0.0055676 & 0.0000000 \\
\hline NGC6440 & 10.214 & 16.779 & 2.1937630 & -0.0479402 & -0.0058459 & 0.0002235 \\
\hline NGC6441 & 10.532 & 17.612 & 2.3822459 & -0.0921838 & -0.0049885 & 0.0002610 \\
\hline NGC6453 & 10.622 & 16.802 & 2.4814560 & -0.2045837 & 0.0056062 & 0.0000000 \\
\hline NGC6522 & 9.863 & 16.009 & 4.9753892 & -0.7589820 & 0.0455941 & -0.0009510 \\
\hline NGC6528 & 9.338 & 16.265 & 2.0592060 & -0.1242321 & 0.0022911 & 0.0000000 \\
\hline NGC6553 & 8.499 & 15.715 & 2.2585166 & -0.1573739 & 0.0038187 & 0.0000000 \\
\hline NGC6558 & 10.079 & 16.537 & 2.9453618 & -0.2744620 & 0.0077358 & 0.0000000 \\
\hline NGC6569 & 10.471 & 17.562 & 2.1521419 & -0.0924727 & -0.0036560 & 0.0002197 \\
\hline NGC6624 & 9.536 & 16.365 & 2.3521783 & -0.1859118 & 0.0046656 & 0.0000000 \\
\hline M28 & 8.825 & 15.205 & 4.4994024 & -0.7116985 & 0.0437171 & -0.0009187 \\
\hline M69 & 9.574 & 16.907 & 1.3614957 & 0.0510122 & -0.0142585 & 0.0004771 \\
\hline NGC6638 & 9.936 & 16.999 & 1.7881424 & -0.0704574 & -0.0028577 & 0.0001619 \\
\hline NGC6642 & 10.023 & 17.402 & 2.2953396 & -0.1858459 & 0.0048425 & 0.0000000 \\
\hline M22 & 7.737 & 14.092 & 1.6818249 & -0.1488596 & 0.0044827 & 0.0000000 \\
\hline
\end{tabular}




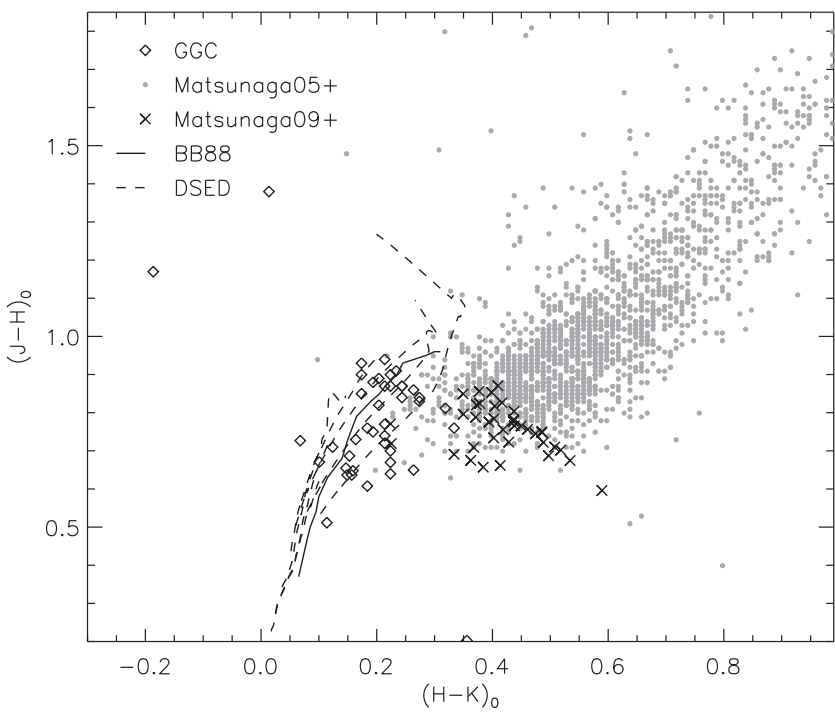

Figure 7. Near-IR colour-colour diagram showing dereddened colours of periodic AGB variables in several GGCs from Lebzelter \& Wood (2011) and Sahay et al. (2014) as diamonds, as well as Mira variables towards the Galactic Centre from Matsunaga et al. $(2005,2009)$ as filled grey circles and crosses respectively. K and M giant colours from Bessell \& Brett (1988) are overplotted as a solid line, and predictions of 12 Gyr DSED models for $[\mathrm{Fe} / \mathrm{H}]=-2.5$ to +0.5 (increasing from left to right on the plot) as dashed lines.

RGB and AGB variables may be more easily detected owing to generally larger pulsational amplitudes (Kiss \& Bedding 2003, 2004), lower amplitude RGB stars pulsate as well, with amplitudes ranging from hundreths of magnitudes for the OGLE Small Amplitude Red Giants in the Magellanic Clouds (OSARGs; Soszyński et al. 2004) and the Galactic bar (Wray, Eyer \& Paczyński 2004) down to millimagnitudes for low-luminosity RGB stars (e.g. Bedding et al. 2010).

Disconcertingly, the success of recent variability campaigns targeted at luminous GGC members at both optical (e.g. Layden et al. 2010; Sahay et al. 2014; Abbas et al. 2015) and IR wavelengths (Matsunaga, Fukushi \& Nakada 2006; Sloan et al. 2010) implies that the current census of variable upper RGB/AGB stars in GGCs is likely incomplete, as these stars are often saturated in photometric time series investigations of less luminous RR Lyrae and SX Phoenicis pulsators. Moreover, even when variability data are available, it remains unclear to what extent pulsational properties aid in separating AGB from RGB members near the TRGB, especially when only a small number of time series epochs are available. On one hand, fig. 4 of Lebzelter \& Wood (2005) as well as the results of Sahay et al. (2014) suggest that variability amplitude decreases with decreasing luminosity, although with a relatively small sample size and sparse time sampling, the evolutionary state of any individual case may still be unclear. Perhaps, the most useful link between pulsational properties and evolutionary state for luminous giants was illustrated using a combination of photometry and extensive time series data. To this end, Kiss \& Bedding (2003, 2004) found that a significant fraction of the variables below the TRGB are RGB rather than AGB stars. In addition, Soszyński et al. (2004) managed to efficiently separate RGB and AGB stars below the TRGB using detailed pulsational properties, revealing that RGB (e.g. non-AGB) pulsators tend to have almost exclusively short primary periods $(P \lesssim 60 \mathrm{~d}$; see their fig. 8$)$ and small pulsational amplitudes $\left(A_{I}<\right.$ $0.14 \mathrm{mag}$ ). On the other hand, Lebzelter et al. (2005) discuss the difficulty of separating AGB and RGB stars using pulsational properties. While small-amplitude variables below the TRGB appear to be dominated by RGB stars in a statistical sense, an AGB status may be difficult to exclude on any individual case-by-case basis, at least when very high-quality time series data are lacking.

The time series aspect of VVV imaging unfortunately cannot provide any clues with respect to our target cluster TRGBs, as our VVV PSF photometry saturates $>1$ mag below the TRGB. Therefore, given the complexities associated with choosing a single star to represent the location of the TRGB, we provide a detailed clusterby-cluster description of our choice of TRGB star in Appendix A, and list the corresponding TRGB magnitudes in Table A1 without formal uncertainties. A comparison between our TRGB magnitudes and those reported in the literature is given in Section 3.5, and we discuss empirical constraints on the precision of TRGB measurements in Section 4.6, and the impact of the TRGB uncertainty on RGB slope measurements in Section 4.4.1. Lastly, one possibility for definitively separating bright RGB and AGB members in the absence of high-quality time series data may be via spectroscopy (Mészáros, Dupree \& Szalai 2009).

\subsection{The RGBB}

The RGBB in the RGB LF was originally described by Iben (1968) and Thomas (1967), and the investigation of Fusi Pecci et al. (1990) was one of the earlier studies to quantify the relationship between the RGBB luminosity and the chemical abundances of cluster stars. Empirical relations between cluster metallicity and the RGBB luminosity have been presented in optical (e.g. Nataf et al. 2013) as well as near-IR (Cho \& Lee 2002; Valenti et al. 2004b) bandpasses, and we measure the location of the bump in all three $J H K_{S}$ filters. While we defer a discussion of the bump luminosity (and consequently of the GGC distance scale, but see Cohen et al. 2015) to a forthcoming study, we demonstrate below in Section 4.5 that an accurate characterization of the bump apparent magnitude, in combination with other features among luminous, evolved cluster members such as the HB and TRGB, can yield distance- and reddening-independent cluster metallicities with a useful precision.

\subsubsection{Measuring the bump location}

To quantify the location of the RGBB in our target GGCs and its uncertainty, we construct the LF of the RGB using only stars in Sample A. In an attempt to maintain self-consistency in our analysis, the LF is built with a bin size of 0.3 mag for all target clusters, although we found that the use of bin sizes from 0.2 to 0.4 mag had a negligible effect on the resulting RGBB magnitudes compared to their uncertainties. To mitigate the effects of binning, 10 histograms are constructed per cluster, but with the bin starting points shifted fractionally each time by an increment 0.1 times the bin width, and the 10 histograms are then averaged (e.g. Gullieuszik et al. 2007). The resulting LF is then fit with an exponential plus Gaussian (Nataf et al. 2011, 2013) as a function of apparent magnitude $m$ in each filter:

$$
\begin{aligned}
N(m)= & A \exp \left[B\left(m-m_{\mathrm{RGBB}}\right)\right] \\
& +\frac{N_{\mathrm{RGBB}}}{\sqrt{2 \pi} \sigma_{\mathrm{RGBB}}} \exp \left[-\frac{\left(m-m_{\mathrm{RGBB}}\right)^{2}}{2 \sigma_{\mathrm{RGBB}}^{2}}\right] .
\end{aligned}
$$

Here, $A$ is a scalefactor, $B$ gives the exponential slope of the RGB and $m_{\mathrm{RGBB}}$ is the magnitude of the bump. An example of an observed LF and the resulting fit is shown in Fig. 8. 

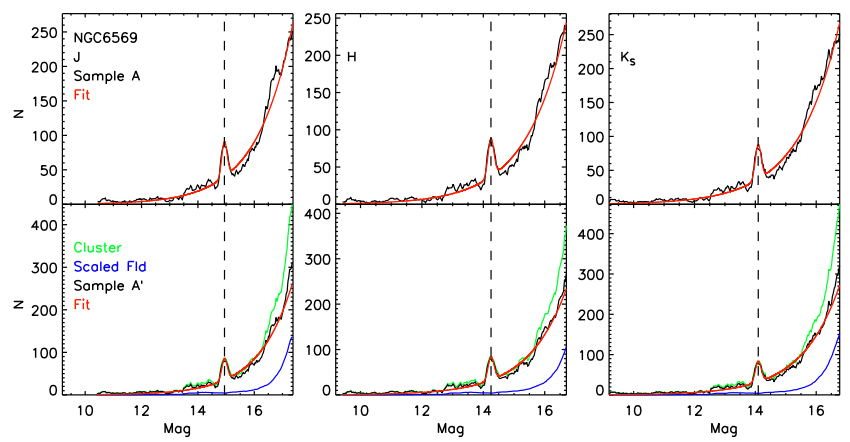

Figure 8. An example of the observed LFs for NGC 6569 in all three $J H K_{S}$ filters (left to right). In the upper panels, the LF constructed from the processed CMDs (Sample A) is shown in black, and the exponential plus Gaussian fit obtained using equation (1) is shown in red. In the lower panels, we show the LFs constructed using Sample $A^{\prime}$, in which the LF is built using all stars in the cluster region (green), the LF of the comparison field is constructed using an identical CMD region and scaled to the spatial area of the cluster region (blue). This scaled field LF is then subtracted to yield a field-subtracted cluster LF (black), shown with the corresponding exponential plus Gaussian fit (red). In all panels, the RGBB magnitude resulting from the fits is shown as a vertical dashed line. RGB LFs, as shown for this example case, are included for all target clusters in the supplementary figures.
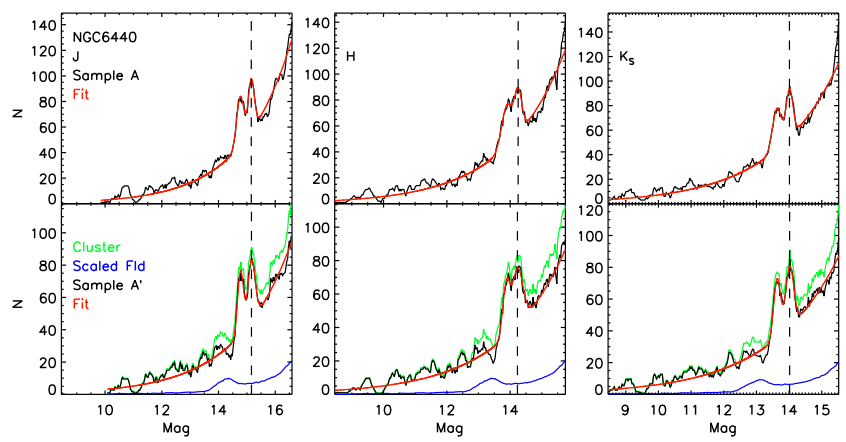

Figure 9. As for Fig. 8, but illustrating an exponential plus double Gaussian fit due to the intrusion of the HB on the RGB LF.

There are four cases where the HB intrudes on the RGB LF due to residual small-scale differential reddening that is unaccounted for by our maps. In these cases (NGC 6440, NGC 6441, NGC 6528 and NGC 6553), the HB causes a discernible second peak in the RGB LF, so an exponential plus double Gaussian is fit (e.g. Nataf et al. 2011):

$$
\begin{aligned}
N(m)= & A \exp \left[B\left(m-m_{\mathrm{RGBB}}\right)\right] \\
& +\frac{N_{\mathrm{RGBB}}}{\sqrt{2 \pi} \sigma_{\mathrm{RGBB}}} \exp \left[-\frac{\left(m-m_{\mathrm{RGBB}}\right)^{2}}{2 \sigma_{\mathrm{RGBB}}^{2}}\right] \\
& +\frac{N_{H B}}{\sqrt{2 \pi} \sigma_{\mathrm{HB}}} \exp \left[\left(-\frac{\left(m-m_{\mathrm{HB}}\right)^{2}}{2 \sigma_{\mathrm{HB}}^{2}}\right)\right] .
\end{aligned}
$$

An example of an exponential plus double Gaussian fit is shown in Fig. 9.

Because we employ the entire magnitude range of the RGB for our exponential plus Gaussian fits rather than a restricted magnitude range around the RGBB (e.g. Nataf et al. 2013; Calamida et al. 2014), the resulting RGBB magnitudes are robust to both gaps in the LFs of the processed CMDs as well as stochastic fluctuations at the bright end of the LFs due to the exponential nature of the RGB LF.

\subsubsection{Quantifying Uuncertainties}

To calculate the total uncertainty on the bump magnitudes resulting from the fit, we take our multibinning approach as well as the photometric errors into account using bootstrap resampling in each cluster. For each of 1000 Monte Carlo iterations, all stars are offset in the CMD by a random amount drawn from a Gaussian distribution that has a standard deviation equal to their photometric error. The entire fitting procedure is then repeated, including the multibin generation of the LF and the exponential plus Gaussian fits, and the resulting bump magnitudes are reported for each iteration. To be conservative, the uncertainty that we report for each parameter is the quadrature sum of the reported uncertainty from the fit to the observed LF plus the standard deviation of the 1000 best-fitting values output from the bootstrapping iterations. Furthermore, if the observed value of a parameter is deviant from the median of the 1000 values output by the bootstrapping procedure by more than this standard deviation, it is considered dubious, indicated by parentheses in Table 3.

To test whether the measurement of the RGBB magnitude is affected by discontinuities or other artefacts of imperfect field star decontamination that may be present in the processed CMDs seen in Figs 4 and 5, we have redetermined the RGBB magnitudes using an alternate procedure. Rather than constructing the LF from the processed CMDs, we directly decontaminate the LF itself. A multibin LF is generated employing the stars in the CMD region occupied by Sample A, but using all stars in the cluster region before the decontamination procedure was applied. Next, another multibin LF is constructed from the same CMD area, but using only stars physically located in the comparison region (e.g. outside the cluster tidal radii). This comparison region LF is scaled to the relative area of the cluster region and subtracted from the cluster region LF, again performing 1000 Monte Carlo iterations where the comparison and cluster stars are offset by Gaussian deviates of their photometric errors. A comparison between the RGBB magnitudes obtained from this alternate procedure, that we refer to as Sample A', versus those obtained above from Sample A, is shown in Fig. 10. The mean offset in each filter between the RGBB magnitude from Sample A and Sample $A^{\prime}$ is given in each panel of Fig. 10 along with the standard deviation of the mean, revealing a mean offset of $<0.02$ mag in all three filters. Furthermore, the uncertainties in the RGBB determined using Sample $A^{\prime}$ are not larger than those determined from Sample A, and the ratio of the RGBB uncertainties measured from the two samples has a median of 1 in all three filters. Given the generally smoother LFs and more stable fits to sample $\mathrm{A}^{\prime}$ as compared to Sample A, we adopt the RGBB magnitudes resulting from the fits to Sample A'. However, both sets of RGB LFs as presented in Figs 8 and 9 are included for all target clusters in the supplementary figures, along with cluster CMDs zoomed on the RGBs. Finally, while a detailed study of other RGBB parameters (i.e. number counts, radial gradients, and skewness) is better performed with high spatial resolution, completeness-corrected photometry, the values of the LF exponent $B$ that we obtain from Sample $\mathrm{A}^{\prime}$ are $B=(0.63,0.59) \pm(0.11,0.12)$ in $J$ and $K_{S}$, respectively, in reasonable $(<1 \sigma)$ agreement with values found in the $I$ band by Nataf et al. (2013).

The magnitudes of the RGBB in each filter for all of our target clusters are listed in Table 3, using parentheses to indicate uncertain 


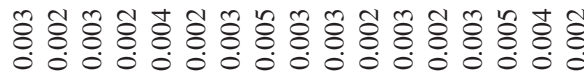
H H H H H H H H H H H H H H H H H

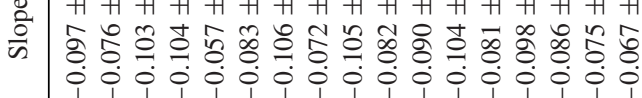

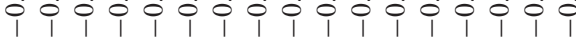

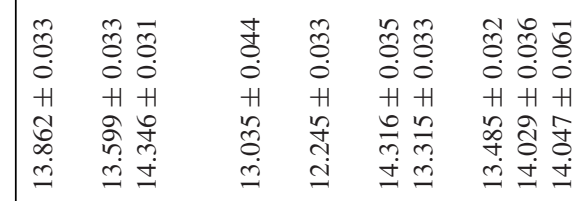

\begin{tabular}{|c|c|c|c|c|c|}
\hline & & & & & \\
\hline$\stackrel{m}{0}$ & 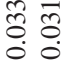 & $\stackrel{\Xi}{\Xi}$ & 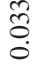 & $\begin{array}{ll}n & m \\
0 & 0 \\
0 & 0\end{array}$ & $\begin{array}{lll}2 & 0 & 0 \\
0 & 0 & 0 \\
0 & 0 & 0 \\
0\end{array}$ \\
\hline$H$ & $H+H$ & $H$ & $H$ & $H+H$ & $H+H$ \\
\hline ले & $\vec{\infty} \stackrel{\infty}{\dot{\infty}}$ & ర్ల & $\stackrel{\infty}{+}$ & 合 & ㅇํำ \\
\hline$\dot{\Xi}$ & $\dot{\theta} \dot{I}$ & $\dot{\sigma}$ & ㄱ & $\dot{\Xi}$ & $\ddot{g} \dot{\square}$ \\
\hline & $m \vec{m}$ & 寸 & $m$ & & \\
\hline & 00 & ㅁ․ & 0 & 0 & 0 \\
\hline 0 & $\dot{0} 0$ & 0 & 0 & 00 & $\dot{0} 0 \dot{0}$ \\
\hline$H$ & $H H$ & $H$ & $H$ & $H+H$ & $H H H$ \\
\hline$\stackrel{n}{\ddagger}$ & 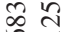 & 0 & I & ○ $\infty$ & n $n$ n \\
\hline gे & 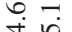 & gे & $\stackrel{n}{n}$ & aे & 我 \\
\hline & $\simeq$ & 2 & 2 & \pm 2 & I \\
\hline
\end{tabular}

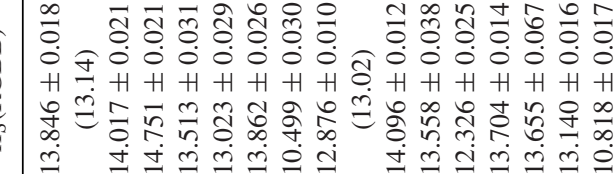

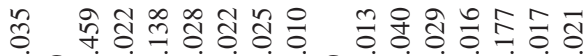

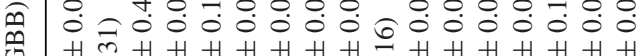

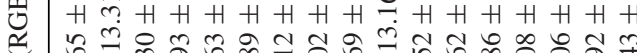

赵

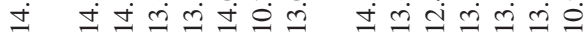

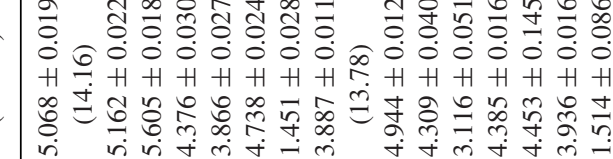

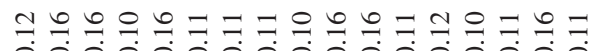

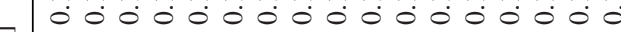

IH H H H H H H H H H H H H H

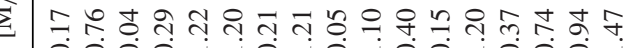

$\begin{array}{llllllllllll}1 & 1 & 1 & 1 & 1 & 1 & 1 & 1\end{array}$

oे

I $H+H+H+H+H+H+H+H+H+H+1$

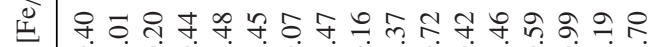

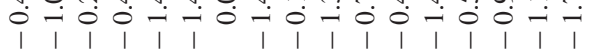

s

宓

-

8 事

$m \approx \pm$
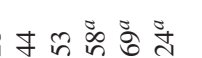

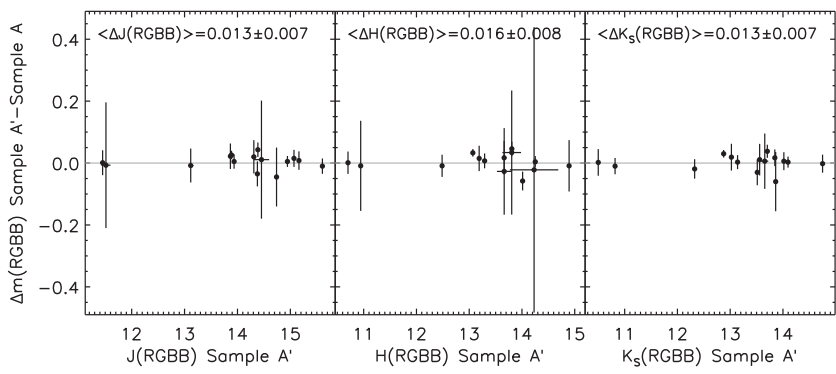

Figure 10. The difference between the RGBB magnitudes measured using Sample A and Sample A', shown as a function of $m$ (RGBB) from Sample $\mathrm{A}^{\prime}$ in all three $J H K_{S}$ filters. The grey horizontal line represents equality, and the mean offset and its standard deviation are given at the top of each panel.

values. The magnitudes of photometric features given in Table 3 are apparent magnitudes, measured using photometry that has been corrected for reddening differentially across each cluster, but has not been corrected for total line-of-sight extinction or distance.

\subsection{HB magnitude}

Various methods have historically been applied to measure the magnitude of the HB and its uncertainty, including the median of a CMD-selected region (e.g. Grocholski \& Sarajedini 2002; Nataf et al. 2013), Gaussian fits to the LF peak (e.g. Calamida et al. 2014), and the location of the maximum of the cluster LF (e.g Valenti et al. 2004a). In the near-IR, an obvious complicating factor is the nearverticality of the HB for less metal-rich clusters, so that an LF peak representative of the HB location is not always detectable for GGCs with exclusively blue HBs (e.g. Cohen et al. 2015). Therefore, for compatibility with previous studies, we restrict our HB analysis to clusters with relatively red HBs with a detectable peak in the LF of the HB, and use the observed cluster LF peak to quantify the location of the $\mathrm{HB}$ in $J H K_{S}$ magnitude. In order to isolate the HB from the influence of the RGBB, we construct the LF using only stars in the processed CMDs in Sample B, that are those lying more than $3 \sigma$ blueward of the cluster fiducial sequences. The LF is built from this sample using the same bin sizes, multibinning, and bootstrap resampling as in the case of the RGBB. However, in lieu of a Gaussian fit to the LF, the reported HB magnitude is simply the magnitude corresponding to the LF peak. This is done both for compatibility with previous near-IR studies (e.g. Valenti et al. 2004a), and because models and data demonstrate that the HB LF may be non-Gaussian in near-IR magnitude (e.g. Salaris et al. 2007, see their fig. 10). Therefore, as in Cohen et al. (2015), the reported uncertainties are the quadrature sum of the standard deviation of the LF peak over the bootstrap iterations plus the effective resolution element of the LF.

We have performed our measurement of the HB LF and its peak neglecting known RR Lyrae variables in our target clusters. To check whether their inclusion affects the measured HB magnitude, we have reperformed our fits (including the bootstrapping iterations) with all known variables included. We found that in all cases, the resultant HB magnitude is unaffected beyond the reported uncertainties, consistent with simulations by Milone et al. (2014) demonstrating that even in optical bandpasses, the influence of RRL photometric variability on single-epoch photometry negligibly affected the HB morphological parameters that they measured.

In order to assess the influence of the decontamination procedure, including potential imperfect subtraction of blue Galactic disc stars on the measured HB magnitudes, we have performed a 


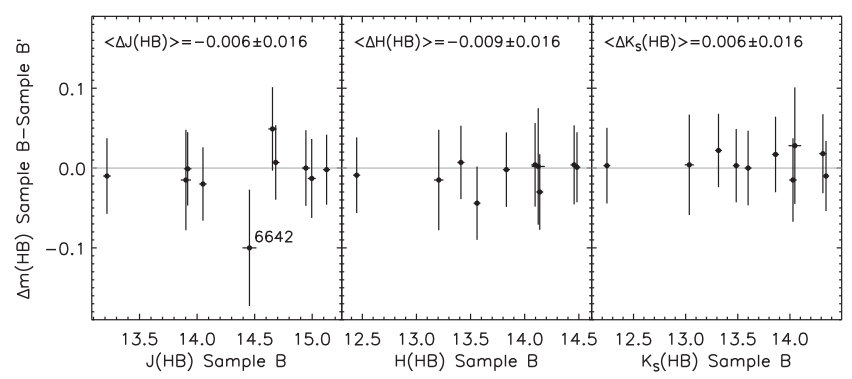

Figure 11. The difference between the HB magnitudes measured using Samples B and B', shown as a function of $m(\mathrm{HB})$ from Sample B in all three $J H K_{S}$ filters. Symbols are as in Fig. 10.

Table 4. Comparison to literature values.

\begin{tabular}{lrc}
\hline Parameter & $\langle$ This study-literature $\rangle$ & $N($ clus $)$ \\
\hline$J($ RGBB $)$ & $-0.045 \pm 0.020$ & 10 \\
$H($ RGBB $)$ & $0.014 \pm 0.027$ & 7 \\
$K_{S}($ RGBB $)$ & $-0.030 \pm 0.020$ & 11 \\
$J(\mathrm{HB})$ & $-0.052 \pm 0.020$ & 9 \\
$H(\mathrm{HB})$ & $0.004 \pm 0.023$ & 7 \\
$K_{S}(\mathrm{HB})$ & $-0.025 \pm 0.020$ & 7 \\
$J($ TRGB $)$ & $0.102 \pm 0.026$ & 14 \\
$H($ TRGB $)$ & $0.113 \pm 0.028$ & 11 \\
$K_{S}($ TRGB $)$ & $0.117 \pm 0.043$ & 16 \\
Slope $_{J K}$ & $-0.0003 \pm 0.0011$ & 11 \\
\hline
\end{tabular}

comparison analogous to Fig. 10. Specifically, the HB LF was generated using all stars in the CMD region occupied by Sample B before statistical decontamination, and a field HB LF was generated from this same CMD area using stars spatially located in the comparison region. The comparison region LF was scaled to the area of the cluster region and subtracted before measuring the peak of the resultant LF over 1000 bootstrapping iterations in which photometric errors were applied to both the cluster and comparison region stars. A comparison between the HB magnitudes measured from this sample, denoted as Sample B' ${ }^{\prime}$, and the HB magnitudes measured from Sample B (using the statistically decontaminated CMD directly) is shown in Fig. 11. This comparison illustrates that the HB magnitudes obtained using the two methods agree to within their uncertainties, with the only slight $(<1.4 \sigma)$ exception of NGC 6642 in the $J$ band, that, in any case, is excluded from the calibration of our photometric metallicity relations (see Section 4).

\subsection{Comparison with literature values}

We compare our observed values listed in Tables 3 and A1 with those from the literature, using the most recent sources as follows: Where available, we use values from the systematic near-IR photometric studies of our target clusters by Valenti et al. (2004a,b) and V10. Otherwise, we take from Chun et al. (2010) the $K_{S}$ values and RGB slope for NGC 6642, the $K_{S}$ magnitude of the RGB bump for NGC 6401 and all available near-IR parameters for M 28. Additionally, the magnitude of the RGB tip in M 22 from 2MASS is taken from Monaco et al. (2004). In Table 4, we list the mean offset between our values and these literature values and its standard deviation, as well as the total number of clusters available for comparison. Bearing in mind that both photometric calibration uncertainties as well as observational measurement uncertainties contribute to this difference, the values are generally in good agreement. Our values for the TRGB magnitude lie $\sim 0.1$ faintward of those re- ported by Valenti et al. (2004b), consistent with the suggestions of both Dalcanton et al. (2012) and Gorski et al. (2016) that the near-IR TRGB magnitude from the Valenti et al. (2004b) calibration is $0.1-0.2 \mathrm{mag}$ too bright. In this case, the discrepancy could be partially due to the exclusion of (then-unknown) AGB variables, although it is well within the margin suggested by measurement error alone: the median published uncertainty of literature TRGB measurements is $0.22 \mathrm{mag}$ in $K_{S}$ and $0.23 \mathrm{mag}$ in $J$ and $H$ (noting that these reported values neglect the additional contribution from uncertainties in the photometric calibration to the 2MASS system), and we revisit empirical constraints on the precision of the near-IR TRGB magnitude in Section 4.6.

\subsection{Some special cases}

There are a few specific cases of clusters for which a single $\mathrm{HB} / \mathrm{RGBB}$ value may not be appropriate that deserve some mention. For the double HBs of NGC 6440 and NGC 6569 reported by Mauro et al. (2012), the values that we obtain are in good agreement, both intermediate between the two HB peaks that they report in each cluster: for NGC 6440, we find $K_{S}(H B)=13.599 \pm 0.033$, in comparison with their values of 13.55 and 13.67 for the two HBs, and for NGC 6569, we obtain $K_{S}(H B)=14.316 \pm 0.035$ in comparison to 14.26 and 14.35 for the two HBs. Since we employ the same data as in that study, we cannot constrain the nature of the HBs beyond the results that they report, and a more detailed study of the HB morphology in these clusters using deep, high-resolution imaging is underway (Mauro et al., in preparation). Our $K_{S}(H B)$ values also agree well with those employed by M14 to devise reduced Ca II equivalent width-[Fe/H] relations: All clusters in common have $K_{S}(\mathrm{HB})$ values that agree to within their uncertainties, with the exception of NGC 6638, for which M14 report a significantly brighter value $(13.70 \pm 0.05$ versus $14.029 \pm 0.036)$. This merely reflects a difference in methodology, since M14 used the reddest part of the $\mathrm{HB}$, as given by theoretical models in combination with distances of V10, to calculate their $K_{S}(\mathrm{HB})$ values, whereas we report the location of the observed LF peak.

We also compare our $K_{S}(\mathrm{HB})$ and $K_{S}(\mathrm{RGBB})$ values to those reported for NGC 6528 by Calamida et al. (2014) using a sample of proper motion-selected cluster members. Their value of $K_{S}$ (bump) $=13.85 \pm 0.05$ compares well with our measurement of $K_{S}$ (bump) $=13.862 \pm 0.026$, and they suggest a double-peaked HB with peaks at $K_{S}=12.97 \pm 0.02$ and $13.16 \pm 0.02$. As we employ some of the same data that they used, we cannot comment further on this feature, but our intermediate value of $K_{S}(H B)=13.035 \pm 0.044$ supports both the location and atypically large width in magnitude that they report for the HB of this cluster. As they cite possible residual field contamination of their proper motion-selected sample as one possible cause of the bimodality, a detailed study of this feature may benefit from high-resolution near-IR imaging of a thoroughly cleaned sample of cluster members (Cohen et al., in preparation).

\section{DISTANCE- AND REDDENING-INDEPENDENT CALIBRATIONS}

Several of the photometric parameters that we have reported can be used to construct indices from relative measurements made on a cluster CMD. By choosing a set of calibrating clusters with wellmeasured metallicities, we can build relations between photometric indices versus metallicity that may be applied as distance- and reddening-independent metallicitity indicators. The relative photometric indices that we explore include the slope of the RGB in the 
Table 5. $[\mathrm{Fe} / \mathrm{H}]$ and $[\mathrm{M} / \mathrm{H}]$ values for target clusters.

\begin{tabular}{|c|c|c|c|c|c|c|c|}
\hline Cluster & {$[\mathrm{Fe} / \mathrm{H}](\mathrm{C} 09)$} & {$[\alpha / \mathrm{Fe}]$} & Reference & {$[\mathrm{M} / \mathrm{H}]$} & {$[\mathrm{Fe} / \mathrm{H}](\mathrm{M} 14)$} & {$[\mathrm{Fe} / \mathrm{H}](\mathrm{HiRes})$} & Reference \\
\hline${\mathrm{NGC6} 380^{a}}^{a}$ & $-0.40 \pm 0.09$ & & & $-0.17 \pm 0.12$ & $-0.72 \pm 0.11$ & & \\
\hline NGC6401 & $-1.01 \pm 0.14$ & & & $-0.76 \pm 0.16$ & & $-1.10 \pm 0.20$ & 1 \\
\hline $\mathrm{NGC6440}^{a}$ & $-0.20 \pm 0.14$ & 0.34 & 2 & $0.04 \pm 0.16$ & $-0.41 \pm 0.11$ & $-0.57 \pm 0.02$ & 2 \\
\hline NGC6441 & $-0.44 \pm 0.07$ & 0.21 & 2 & $-0.29 \pm 0.10$ & $-0.65 \pm 0.11$ & $-0.57 \pm 0.02$ & 2 \\
\hline NGC6453 & $-1.48 \pm 0.14$ & & & $-1.22 \pm 0.16$ & & & \\
\hline $\mathrm{NGC6522}^{a}$ & $-1.45 \pm 0.08$ & 0.35 & 3 & $-1.20 \pm 0.11$ & $-1.38 \pm 0.12$ & $-1.08 \pm 0.13$ & 3 \\
\hline NGC6528 ${ }^{a}$ & $0.07 \pm 0.08$ & 0.20 & 4,5 & $0.21 \pm 0.11$ & $-0.24 \pm 0.11$ & $-0.24 \pm 0.19$ & 6 \\
\hline NGC6544 & $-1.47 \pm 0.07$ & & & $-1.21 \pm 0.11$ & $-1.50 \pm 0.12$ & & \\
\hline NGC6553 & $-0.16 \pm 0.06$ & 0.30 & & $0.05 \pm 0.10$ & $-0.13 \pm 0.11$ & $-0.20 \pm 0.15$ & 7 \\
\hline $\mathrm{NGC6558}^{a}$ & $-1.37 \pm 0.14$ & 0.37 & 8 & $-1.10 \pm 0.16$ & $-1.07 \pm 0.11$ & $-0.97 \pm 0.15$ & 8 \\
\hline NGC6569 ${ }^{a}$ & $-0.72 \pm 0.14$ & 0.43 & 9 & $-0.40 \pm 0.16$ & $-1.18 \pm 0.11$ & $-0.90 \pm 0.02$ & 9 \\
\hline NGC6624 $4^{a}$ & $-0.42 \pm 0.07$ & 0.37 & 9 & $-0.15 \pm 0.11$ & $-0.72 \pm 0.12$ & $-0.79 \pm 0.02$ & 9 \\
\hline M28 & $-1.46 \pm 0.09$ & & & $-1.20 \pm 0.12$ & $-1.31 \pm 0.12$ & & \\
\hline M69 & $-0.59 \pm 0.07$ & 0.31 & 10 & $-0.37 \pm 0.10$ & $-0.66 \pm 0.12$ & $-0.77 \pm 0.02$ & 10 \\
\hline NGC6638 & $-0.99 \pm 0.07$ & & & $-0.74 \pm 0.11$ & $-0.95 \pm 0.12$ & & \\
\hline $\mathrm{NGC6642}^{a}$ & $-1.19 \pm 0.14$ & & & $-0.94 \pm 0.16$ & & $-1.40 \pm 0.20$ & 1 \\
\hline M22 & $-1.70 \pm 0.08$ & 0.32 & 11 & $-1.47 \pm 0.11$ & $-1.83 \pm 0.11$ & $-1.75 \pm 0.04$ & 12 \\
\hline
\end{tabular}

${ }^{a}$ Cluster excluded from calibrations in Sections 4.4.1, 4.5 and 4.6 due to uncertain metallicity. References: (1) Minniti (1995), (2) Origlia et al. (2008),

(3) Ness et al. (2014), (4) Zoccali et al. (2004), (5) Origlia et al. (2005), (6) Sobeck et al. (2006), (7) Alves-Brito et al. (2006), (8) Barbuy et al. (2007),

(9) Valenti et al. (2011), (10) Lee (2007), (11) Marino et al. (2011), (12) Mucciarelli et al. (2015).

$K_{S},\left(J-K_{S}\right)$ plane ( slope $\left._{J K}\right)$, as well as the magnitude difference between the $\mathrm{HB}$ and $\mathrm{RGBB}\left(\Delta m_{\mathrm{RGBB}}^{\mathrm{HB}}\right)$ and the magnitude difference between the RGB bump and the tip of the RGB $\left(\Delta m_{\mathrm{TRGB}}^{\mathrm{RGB}}\right)$ in each of the three $J H K_{S}$ bandpasses. While we defer a discussion of calibrations versus absolute magnitude, and hence the GGC distance scale to a forthcoming publication, the distance- and reddeningindependent relations that we derive can serve as quantitative tests of evolutionary models as well as photometric metallicity indicators for old stellar populations.

\subsection{Input metallicities: $[\mathrm{Fe} / \mathrm{H}]$}

We wish to use GGCs with the most reliable spectroscopic abundances to calibrate relations between photometric features and cluster metallicity. While recent large-scale spectroscopic campaigns have vastly increased the number of GGCs with high-quality selfconsistent spectroscopic measurements of both $[\mathrm{Fe} / \mathrm{H}]$ and $[\alpha / \mathrm{Fe}]$ (C09; Carretta et al. 2010; Dias et al. 2016), the issue of spectroscopic metallicities remains complicated with regard to the GGCs located towards the Galactic bulge. In some cases, the values of $[\mathrm{Fe} / \mathrm{H}]$ listed by $\mathrm{C} 09$ are significantly at odds with those from other recent, independent spectroscopic investigations. Therefore, we summarize in Table 5 various spectroscopic $[\mathrm{Fe} / \mathrm{H}]$ values for our target clusters from several sources in addition to C09. These include the near-IR Ca II triplet studies by M14, and an additional set comprised of any independent spectroscopic metallicity measurements in the literature, that we denote as 'HiRes'. These values are further compared in Fig. 12, where we plot $[\mathrm{Fe} / \mathrm{H}]$ from $\mathrm{C} 09$ versus the HiRes values in the top panel. Significant $(>0.3 \mathrm{dex})$ discrepancies are evident, as noted by M14, who devised a set of 'corrected' C09 values (that they denote 'C09c') for clusters where C09 values showed significant discrepancies from other studies. In the bottom panel of Fig. 12, we compare $\mathrm{C} 09[\mathrm{Fe} / \mathrm{H}]$ with the values given by the $\mathrm{Ca}$ II triplet calibrations of M14, employing their best-fitting relations of $\mathrm{Ca}$ II equivalent width versus $\mathrm{C} 09 \mathrm{c}$ $[\mathrm{Fe} / \mathrm{H}]$ values, that are cubic in the case of the Saviane et al. (2012) equivalent widths (column IIIa of their table 3) and quadratic in the case of the Rutledge, Hesser \& Stetson (1997) equivalent

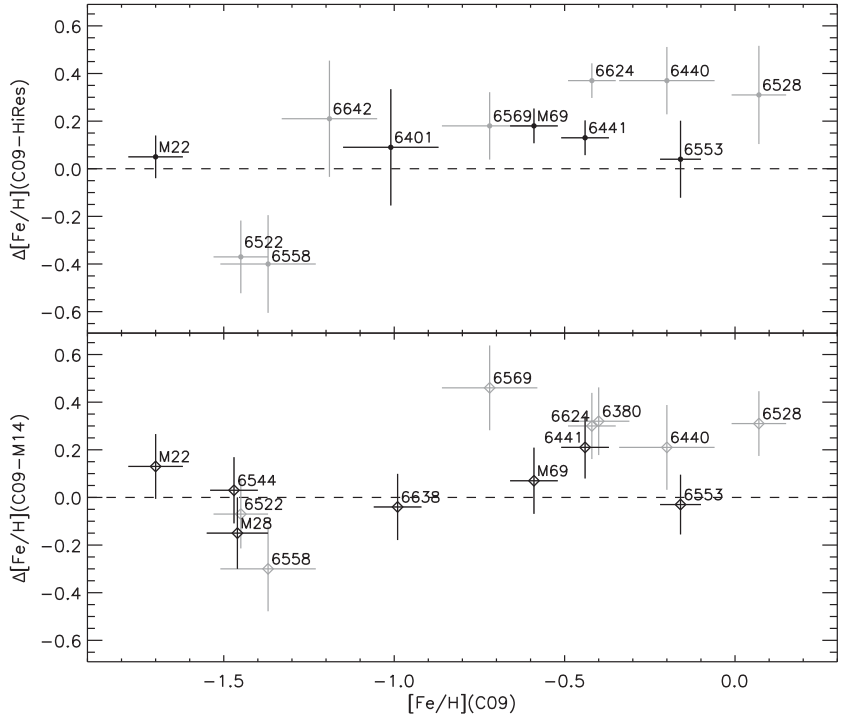

Figure 12. Comparison between $[\mathrm{Fe} / \mathrm{H}]$ values reported by $\mathrm{C} 09$, versus those from independent spectroscopic studies (top panel) as well as the Ca II triplet calibration of M14 (bottom panel). Clusters included as calibrators are plotted in black, and those excluded due to controversial $[\mathrm{Fe} / \mathrm{H}]$ values are plotted in grey. The dotted horizontal line in each panel represents equality, and clusters are labelled by NGC or Messier number.

widths (column IIa of their table 6). The uncertainties on the M14 $[\mathrm{Fe} / \mathrm{H}]$ values employed in Table 5 and Fig. 12 are the unbiased rms that they report from the applicable calibration (evaluated considering only the calibrating clusters), and for the two clusters with equivalent width measurements from both Saviane et al. (2012) and Rutledge, Hesser \& Stetson (1997) (NGC 6528 and NGC 6553), we use the $[\mathrm{Fe} / \mathrm{H}]$ values resulting from the calibration employing the more recent Saviane et al. (2012) equivalent widths.

Given the evident discrepancies in $[\mathrm{Fe} / \mathrm{H}]$ for some clusters, we adopt the following strategy: clusters with controversial $[\mathrm{Fe} / \mathrm{H}]$ values, plotted in grey in Fig. 12 , are excluded from our 
calibrations of photometric indices versus metallicity, and we later use our results to comment on the metallicities of these clusters. The remainder of our VVV targets, plotted in black in Fig. 12, are used to calibrate photometric metallicity indicators, together with recent literature results. Meanwhile, a few of the cases listed in Table 5 deserve further comment regarding their HiRes $[\mathrm{Fe} / \mathrm{H}]$ values as they have been subjected to multiple recent spectroscopic investigations.

NGC 6522: spectroscopic analyses were recently presented by both Ness, Asplund \& Casey (2014) and Barbuy et al. (2014), targeting eight and four giants, respectively. Despite having several stars in common, the two studies report mean $[\mathrm{Fe} / \mathrm{H}]$ values that differ by 0.2 dex, albeit with an uncertainty of $0.15 \mathrm{dex}$ in both cases. In addition, Ness, Asplund \& Casey (2014) find that the cluster is significantly $\alpha$-enhanced, while Barbuy et al. (2014) claim only low to moderate enhancements of $\mathrm{Si}, \mathrm{Ca}$ and $\mathrm{Ti}$. We choose to adopt the abundances of Ness et al. (2014) due to their larger sample size, but we recalculate the mean $[\mathrm{Fe} / \mathrm{H}]$ excluding star B-108 as Barbuy et al. (2014) found that it is blended, bringing the two studies into agreement at the $1 \sigma$ level. While we cannot exclude the possibility that any of the three stars in the Ness et al. (2014) sample not studied by Barbuy et al. (2014) is likewise affected by blending, there is no obvious indication among the reported radial velocities or abundances that this is the case.

NGC 6528: as discussed by M14 and Dias et al. (2016), several recent spectroscopic studies have found $[\mathrm{Fe} / \mathrm{H}]$ values lower than the supersolar value of $[\mathrm{Fe} / \mathrm{H}]=+0.07 \pm 0.08$ from Carretta et al. (2001) used in the compilation of C09. Origlia, Valenti \& Rich (2005) report $[\mathrm{Fe} / \mathrm{H}]=-0.17 \pm 0.01$ from high-resolution near-IR spectra of four RGB stars, while Zoccali et al. (2004) and Sobeck et al. (2006) report $[\mathrm{Fe} / \mathrm{H}]=-0.1 \pm 0.2$ and $-0.24 \pm 0.19 \mathrm{dex}$, respectively, from high-resolution optical spectra of three stars (one HB star and two RGB stars). Both of these values are in good agreement with the low-resolution optical spectra of 17 stars by Dias et al. (2015), who report $[\mathrm{Fe} / \mathrm{H}]=-0.13 \pm 0.05$, and we adopt the estimate of Sobeck et al. (2006) for the HiRes set of $[\mathrm{Fe} / \mathrm{H}]$ values and comment further on photometric constraints in Section 5.

M 22 (NGC 6656): several spectroscopic investigations have claimed a split/multimodality in [Fe/H] (Marino et al. 2011, 2012; Alves-Brito et al. 2012; Marino, Milone \& Lind 2013). However, Mucciarelli et al. (2015) found that when Fe I lines, that are more vulnerable to non-local thermodynamic equilibrium effects, are excluded, Fe II lines show no significant spread in iron abundance. For our purposes, this turns out to be somewhat of a moot point, since the value that Mucciarelli et al. (2015) calculate from Fe II lines, $[\mathrm{Fe} I \mathrm{I} / \mathrm{H}]=-1.75 \pm 0.04$, is in good agreement with the $\mathrm{C} 09$ value of $[\mathrm{Fe} / \mathrm{H}]=-1.70 \pm 0.08$, so we include this cluster in our set of calibrators. $^{10}$

\subsection{The global metallicity $[\mathrm{M} / \mathrm{H}]$}

Since models and observations both suggest that the upper RGB is sensitive to variations in $[\alpha / \mathrm{Fe}]$ as well as $[\mathrm{Fe} / \mathrm{H}]$ in the near-IR (Cohen et al. 2015), we build relations in terms of both $[\mathrm{Fe} / \mathrm{H}]$ and the global metallicity $[\mathrm{M} / \mathrm{H}]$, defined by Salaris, Chieffi \&

\footnotetext{
${ }^{10}$ Incidentally, these $[\mathrm{Fe} / \mathrm{H}]$ values are not, on average, inconsistent with other high-resolution studies. Despite reporting a bimodality in $[\mathrm{Fe} / \mathrm{H}]$, the spectroscopic study of 35 RGB stars by Marino et al. (2011) gives a mean value of $[\mathrm{Fe} / \mathrm{H}]=-1.77 \pm 0.03 \mathrm{dex}$.
}

Straniero $(1993)$ as $[\mathrm{M} / \mathrm{H}]=[\mathrm{Fe} / \mathrm{H}]+\log \left(0.638 f_{\alpha}+0.362\right)$, where $f_{\alpha}=10^{[\alpha / \mathrm{Fe}]}$. For clusters without spectroscopic measurements of $[\alpha / \mathrm{Fe}]$, we assume the linear $[\alpha / \mathrm{Fe}]-[\mathrm{Fe} / \mathrm{H}]$ relation of Nataf et al. (2013), and conservatively assume $\sigma[\alpha / \mathrm{Fe}]=0.1 \mathrm{dex}$. This yields $0.3<[\alpha / \mathrm{Fe}]<0.4$ for all target clusters without literature values of $[\alpha / \mathrm{Fe}]$, in accord with spectroscopic measurements of $\alpha$-enhancement found in GGCs towards the Galactic bulge (Origlia, Rich \& Castro 2002; Origlia et al. 2005; Barbuy et al. 2007; Origlia, Valenti \& Rich 2008; Valenti, Origlia \& Rich 2011; Valenti et al. 2015). In the case of NGC 6528, Zoccali et al. (2004) find $[\alpha / \mathrm{Fe}]$ $\sim 0.1$, whereas Origlia et al. (2005) report $[\alpha / \mathrm{Fe}] \sim 0.33$, so we assume $[\alpha / \mathrm{Fe}]=0.2 \pm 0.1$. In all cases, we calculate the uncertainty in the resulting $[\mathrm{M} / \mathrm{H}]$ following equation 7 of Nataf et al. (2013), ${ }^{11}$ and the values of $[\alpha / \mathrm{Fe}]$ and their sources as well as the resulting $[\mathrm{M} / \mathrm{H}]$ for our VVV target clusters are listed in Table 5.

\subsection{Extending the calibration baseline}

Although our target clusters span a reasonably broad range in metallicity, they suffer from the limitation that there are no GGCs included that are more metal-poor than M $22([\mathrm{Fe} / H] \lesssim-1.7)$. In order to maximize the applicable metallicity range of our calibrations as well as increase the sample size, we supplement the values that we measure with those available in the literature that have high-quality spectroscopic abundances (C09; Carretta et al. 2010). For this purpose, we denote the target clusters described thus far as the ' $\mathrm{VVV}$ ' sample (including NGC 6544, as described in Cohen et al. 2014), and supplement them with near-IR measurements of 12 optically well-studied GGCs from Cohen et al. (2015; the 'ISPI' sample), as well as the data base of near-IR GGC photometry from V10 and references therein, designated the 'V10' sample. ${ }^{12}$ For convenience, we have compiled measured photometric features from these supplementary sources in Table 6 .

We now describe the construction of relations between cluster metallicity versus several relative photometric indices that can be measured from cluster CMDs. The indices studied typically span a colour range of $\Delta\left(J-K_{S}\right) \lesssim 0.5$, so in addition to being independent of distance and reddening, they are insensitive to photometric zeropoint uncertainties and the assumed reddening law.

\subsection{RGB Slope (slope $\left.{ }_{J K}\right)$}

\subsubsection{Observed slope measurements}

A linear relation has traditionally been used to describe cluster metallicity (in terms of $[\mathrm{Fe} / \mathrm{H}]$ and/or $[\mathrm{M} / \mathrm{H}]$ ) versus the slope of the upper RGB (in terms of colour as function of magnitude), calculated over a magnitude range on the upper RGB where the effects of metallicity variations are most prominent (e.g. Valenti et al. 2004a). The definition of this magnitude range is based on the observation of Kuchinski et al. (1995) that using stars in the range of 0.6-5.1 mag brighter than the zero-age horizontal branch (ZAHB) serves to avoid the influence of HB stars at the faint end and bright AGB variables close to the RGB tip. However, defining a ZAHB magnitude for metal-poor clusters in the near-IR is difficult

\footnotetext{
${ }^{11}$ Where not given explicitly, we calculate $\alpha$ as the mean of $\mathrm{Ti}, \mathrm{Si}, \mathrm{Mg}$ and $\mathrm{Ca}$ (e.g. Valenti et al. 2011) weighted by the inverse squares of their uncertainties.

${ }^{12}$ For values listed without uncertainties by V10, we conservatively assume an uncertainty of 0.005 on the RGB slope and 0.1 mag on the HB and RGB bump magnitudes based on the errors reported by Valenti et al. (2004a,b).
} 


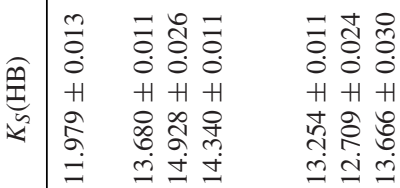

$\therefore \quad ㅇ$

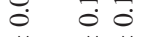

H H H

의 $=\frac{1}{6}$

$m \quad \pm m$

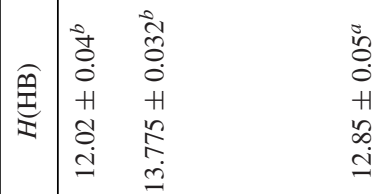

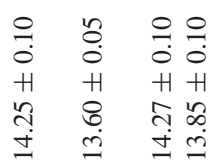

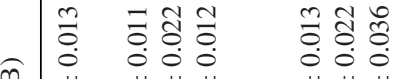

急

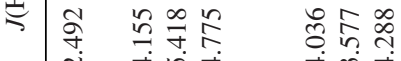

I $\quad \dot{2}$

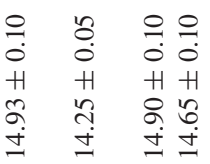

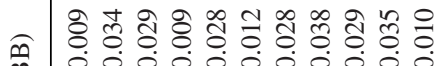

ㄴํำ ㄴํㅇำ

0.0

H H H H H H

$\therefore$ 은요 우

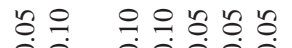

00 O 00000

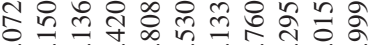

im

n $\mathrm{H} H \mathrm{H} H \mathrm{H}$

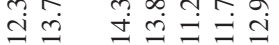

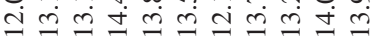

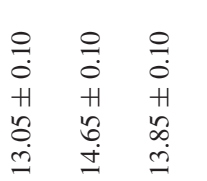

으요 용

$\circ \circ 0$

$+\mathrm{H}+\mathrm{H}$

ิิ

氝

i

0
$\stackrel{0}{0}$
+1
ले
$\stackrel{-}{2}$

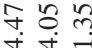

$\dot{I}=\stackrel{9}{=}$

网

券 H H H H H H H H H H H

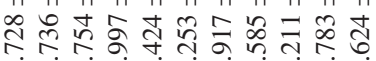

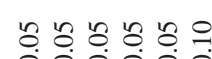

$\begin{array}{lllll}\mathrm{H} & \mathrm{H} & \mathrm{H} & \mathrm{H} & \mathrm{H}\end{array}$

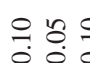

웅웅 ㅇํㅇ 응 H H H H H H H H

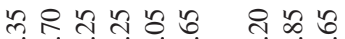

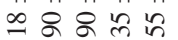
ป

$\ddot{m} \dot{m} \dot{m}$

넌

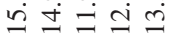

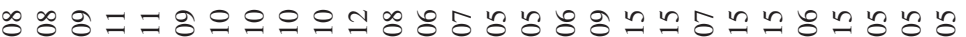
:

I $H H+H+H+H+H+H+H+H+H+H+H+H+H+H+H+1$

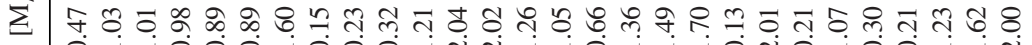

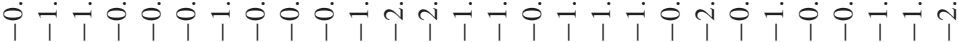

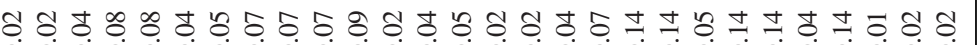

I H H H H H H H H H H H H H H H H H H H H H H H H H H H

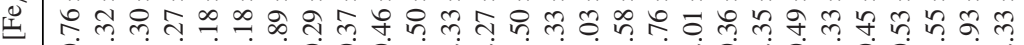

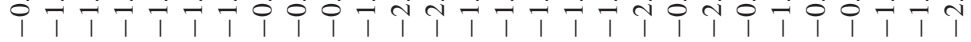




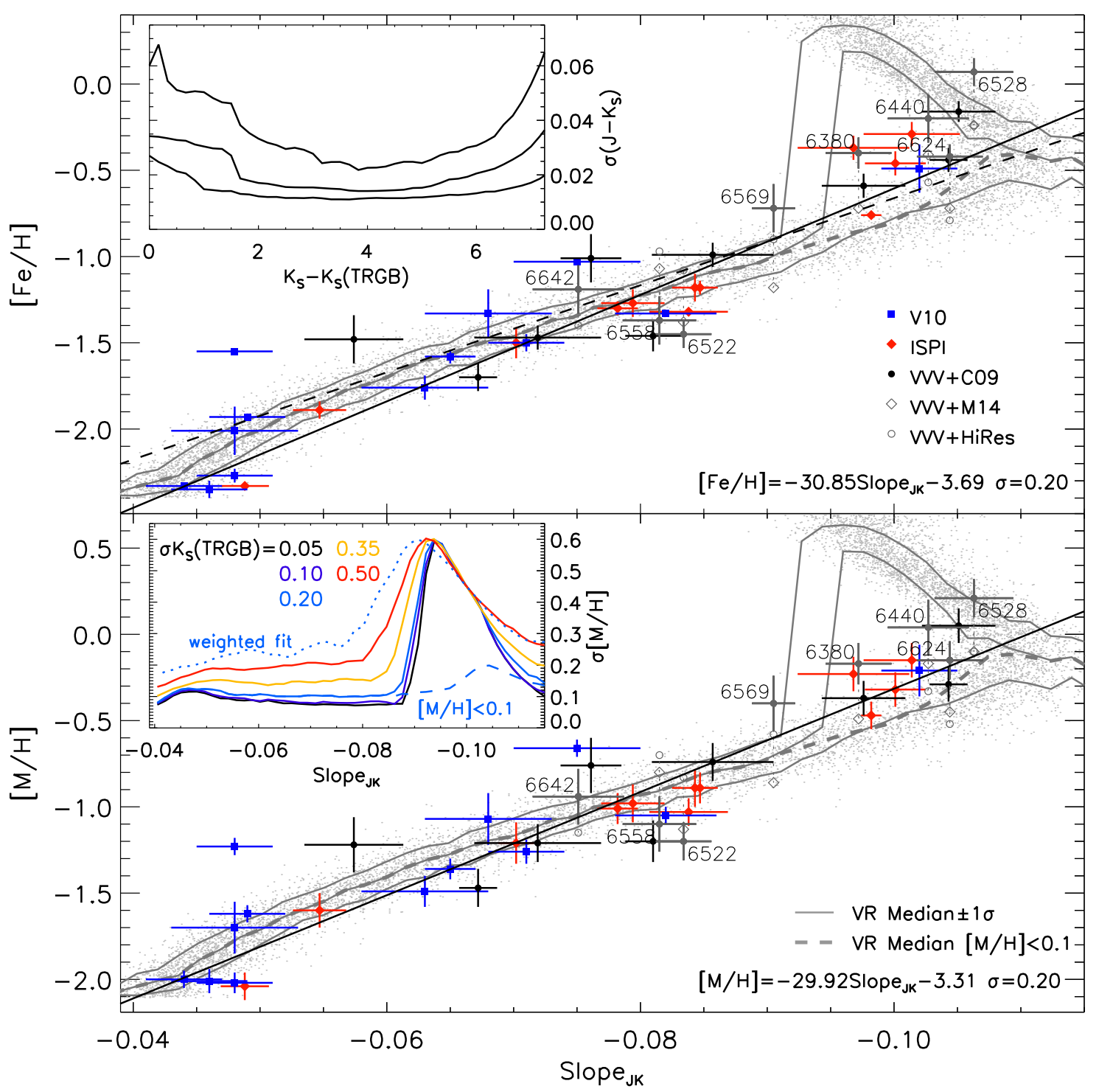

Figure 13. Relations between the slope of the red giant branch and cluster metallicity, in terms of $[\mathrm{Fe} / \mathrm{H}]$ (top) and global metallicity $[\mathrm{M} / \mathrm{H}](\mathrm{bottom})$. In each plot, values for clusters from the VVV sample are shown as filled black circles. Clusters in the VVV sample that are excluded as calibrators due to uncertain metallicities are labelled and plotted in grey rather than black, and their $\mathrm{C} 09$ values are connected by a dotted line to values corresponding to [Fe/H] from M14 (open diamonds) and the HiRes set given in Table 5 (open circles). Additional clusters used as calibrators from the V10 sample (V10, and references therein) are shown as blue squares, and calibrators from Cohen et al. (2015) are shown using red circles. The solid black line represents a least-squares fit to all calibrators (VVV+V10+ISPI) weighted using the uncertainties in metallicity, and the resulting best-fitting equation is given in the bottom right corner of each panel. The dashed black line represents the relation of Valenti et al. (2004a) transformed to the C09 metallicity scale. The curved grey lines represent the median and $\pm 1 \sigma$ values predicted from Monte Carlo simulations using Victoria-Regina evolutionary models (see the text for details), and the individual simulation results are shown as light grey points.

since their HBs are, in fact, almost vertical, so for consistency, we follow the methodology of Valenti et al. (2004a, and references therein) and use the magnitude range of $0.5<\left(K-K_{\mathrm{TRGB}}\right)<5.0$. The RGB slope is measured by fitting a line to all stars that lie in this magnitude range and have colours within $3 \sigma$ of the fiducial sequence (e.g. included in Sample A).

In Fig. 13, we show measured RGB slope values versus both $[\mathrm{Fe} / \mathrm{H}]$ and $[\mathrm{M} / \mathrm{H}]$ (on the $\mathrm{C} 09$ scale). Target clusters from the VVV sample are shown as filled black circles, while those from the literature are shown using blue squares (V04 sample) or red circles (ISPI sample). VVV clusters that were not used as calibrators due to their uncertain $[\mathrm{Fe} / \mathrm{H}]$ values are shown in grey and labelled by NGC number, and for each of these clusters, a vertical dotted line is shown connecting their $\mathrm{C} 09[\mathrm{Fe} / \mathrm{H}]$ values to those from the M14 and HiRes scales as reported in Table 5 (for clarity, error bars on these alternate values are not shown in Fig. 13). As in the case of the HB and RGBB magnitudes, the reported slope errors are the quadrature sum of the formal uncertainty on the slope from an unweighted least-squares fit plus the standard deviation of the slopes obtained over 1000 bootstrapping iterations in which the stars are offset by Gaussian deviates of their photometric errors. Given the difficulties in separating AGB and RGB cluster members discussed in Section 3.2, we have chosen to exclude all known variables from our slope measurements, and the use of a colour cut combined with the exclusion of stars within 0.5 mag of the TRGB serves to effectively remove most known variables from the 
CMD region used to measure the slope. However, in the minority of cases where a small number of variables fall in the CMD region used for slope measurement (one, two and five stars each in M28, M22 and NGC6441, respectively), we have reperformed the slope measurement including these variables and verified that the slopes are unaffected beyond their uncertainties.

\subsubsection{Uncertainties in the slope measurement}

In order to assess the impact of the fitting method, photometric errors and observational uncertainty in the TRGB magnitude, we have performed an additional series of simulations to examine systematic errors on the measurement of the RGB slope. We have generated 1000 synthetic RGBs distributed evenly over the metallicity range of $-2.5<[\mathrm{Fe} / \mathrm{H}]<0.5$ using $12 \mathrm{Gyr} \alpha$-enhanced $([\alpha / \mathrm{Fe}]=0.4)$ Victoria-Regina isochrones (VandenBerg et al. 2014) as these models reasonably reproduce the upper RGB morphology of GGCs in the near-IR (see Cohen et al. 2015 for details). For each iteration, the total number of RGB stars and a value of the LF exponent $B$ were randomly drawn from a Gaussian distribution with the observed mean and standard deviation. Next, all stars were offset using photometric errors randomly drawn from Gaussian distributions with standard deviations equal to the observed median photometric error as a function of magnitude below the TRGB across all of our target clusters, shown in the inset in the upper panel of Fig. 13. Finally, a random measurement uncertainty of 0.2 mag on the TRGB magnitude is added (we explore the choice of this value below), before measuring the RGB slope identically as for the target clusters. In the main panels of Fig. 13, the median and $\pm 1 \sigma$ standard deviation of the slopes from the synthetic RGBs (measured in 50 evenly spaced bins) are shown as curved grey lines.

Because RGBs are typically described in near-IR CMDs using low-order polynomials (e.g. Valenti et al. 2004a) as we have done, it is already known that the use of a line to fit the upper RGB is a first-order approximation (Ferraro et al. 2000), and models predict this. As the upper RGB becomes increasingly negative in slope (less vertical in the $\mathrm{CMD}$ ) at higher metallicities, its curvature increases as well. For this reason, linear fits to the upper RGB become increasingly degenerate at higher $([\mathrm{M} / \mathrm{H}] \gtrsim-0.5)$ metallicities. However, the simulations show that this effect depends entirely on the maximum assumed metallicity limit at the metal-rich end. In other words, the relation between slope and metallicity is no longer monotonic at the metal-rich end (or equivalently slope ${ }_{J K}<-0.09$ ), but this is due entirely to the inclusion of metallicities ranging well above solar in our simulations. To illustrate this conclusion, we have re-evaluated the median and $1 \sigma$ values of metallicity as a function of slope from the simulations, but excluding all iterations with an input $[\mathrm{M} / \mathrm{H}]$ $>-0.1$. The resulting median values are shown as a dotted grey line in the main panels of Fig. 13, illustrating that according to the models, the slope can remain an effective metallicity indicator at high (near-solar) metallicities only if supersolar metallicities can be excluded a priori. However, the data do not show any evidence for such a degeneracy, and, in fact, the more metal-rich calibrating clusters ( slope $_{J K} \leq-0.09$ ) show rms deviations from our linear fit of only 0.15 and 0.12 dex versus $[\mathrm{Fe} / \mathrm{H}]$ and $[\mathrm{M} / \mathrm{H}]$, respectively, as compared to an rms of 0.22 dex versus both $[\mathrm{Fe} / H]$ and $[\mathrm{M} / \mathrm{H}]$ at lower metallicities.

We have also performed sets of simulations to explore two additional sources of uncertainty in the slope measurements. The first of these is the observational uncertainty of the TRGB magnitude. We have reperformed the 1000 -iteration simulation several times, assuming a different observational uncertainty on the location of the TRGB ranging over $\sigma K_{S}(\mathrm{TRGB})=(0.05,0.1,0.2,0.35,0.5)$ mag in each simulation. In the inset in the lower panel of Fig. 13, we plot the median scatter (e.g. standard deviation) in metallicity as a function of slope, colour coded by the input (Gaussian) uncertainty in the TRGB magnitude. These simulations reveal that an uncertainty of up to $\sim 0.2$ mag in the TRGB location does not significantly impact the uncertainty in the inferred metallicity above a lower threshold that is set by the photometric errors and number of available cluster stars typical of our observations. However, if the uncertainty in the TRGB magnitude increases substantially above $\sigma K_{S}(T R G B) \sim 0.2$, the scatter in metallicity inferred from a given slope value is significantly affected, increasing by more than a factor of 2 if the TRGB location is not known to better than $0.5 \mathrm{mag}$.

The second source of systematic uncertainties that can be addressed with such simulations is the use of photometric errors to weight the stars in the relevant CMD region when performing the least-squares fit to measure the slope. An additional series of simulations was performed as described above, assuming $\sigma K_{S}(\mathrm{TRGB})=$ 0.2 to allow a direct comparison, but measuring the slopes by performing a weighted rather than unweighted least-squares fit. The results of this set of simulations are shown as a dotted blue line in the inset in the lower panel of Fig. 13, revealing that the uncertainty of the inferred metallicities increases by a factor of more than 2 when a weighting scheme is used. This result is specific to the distribution of photometric error versus magnitude for our target clusters, and the cause is illustrated in the inset in the upper panel of Fig. 13. The necessity of 2MASS photometry due to saturation in our VVV PSF photometry close to the TRGB causes the photometric errors to increase at the bright end of the magnitude range where the slope is measured. The downweighting of these stars in a photometric error-weighted fit combines with their relative sparseness at the brighter, more poorly populated end of the RGB LF to result in larger scatter in the measured slopes. For this reason, we have employed unweighted least-squares fits when measuring the RGB slopes of our target clusters (also, the use of an unweighted fit is presumably more consistent with previous studies as they do not mention a weighting scheme).

Lastly, we address the influence of uncertainties in the decontamination procedure on our measured slope values. The decontamination procedure gives a formal $1 \sigma$ uncertainty on the number of stars in the cluster region that are probable members, based on both photometric errors as well as Poissonian uncertainties on the number of stars in the cluster and comparison regions. By combining this quantity with the membership probability as a function of location in the cluster CMD, we can check whether any of the stars used in the calculation of the RGB slope have membership probabilities placing them within the $1 \sigma$ error margin of $N_{\text {clus }}$ (given in Table 1). We find that of the stars used to calculate the RGB slope in all target clusters less than 5 per cent of them have membership probabilities placing them within this $1 \sigma$ error margin, and we have verified that the inclusion or exclusion of these stars does not affect the measured slopes beyond their uncertainties. Furthermore, in half of our target clusters, none of the stars used to measure the slope are $1 \sigma$ non-members.

\subsection{HB-mump magnitude difference $\left(\Delta m_{\mathrm{RGBB}}^{\mathrm{HB}}\right)$}

The magnitude difference between the HB and the RGBB is another distance- and reddening-independent metallicity indicator that has not yet been explored in the near-IR. At optical wavelengths, a linear relation was found in the $I$ band between the magnitude of 


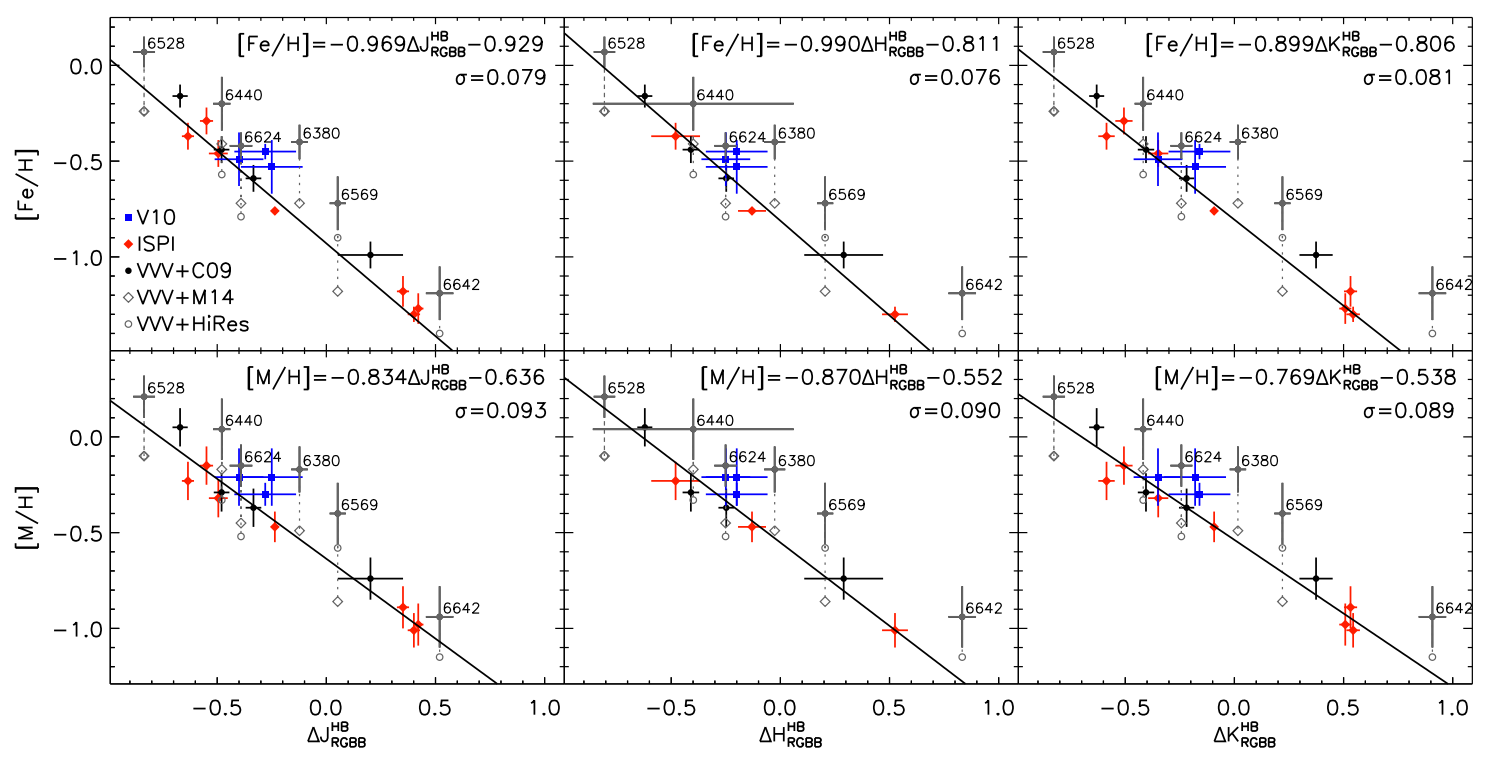

Figure 14. Linear fits in all three $J H K_{S}$ filters to metallicity as a function of the magnitude difference between the RGBB and the HB. Symbols are as in Fig. 13.

the (CMD-selected) red HB and the RGBB magnitude (Nataf et al. 2013). Meanwhile, several other studies (Cassisi \& Salaris 1997; Alves \& Sarajedini 1999; Zoccali et al. 1999; Riello et al. 2003; Di Cecco et al. 2010) have found a somewhat non-linear relation between cluster metallicity and the magnitude difference between the RGBB and the ZAHB in the $V$ band in accord with predictions of evolutionary models. However, among these studies, several different metallicity scales and methodologies for quantifying the ZAHB magnitude were employed.

We perform fits to the magnitude difference between the HB and the RGBB, denoted $\Delta m_{\mathrm{RGBB}}^{\mathrm{HB}}$, as a function of metallicity in all three near-IR $J H K_{S}$ bandpasses. Unlike some of the aforementioned optical studies, the HB magnitude that we employ corresponds to the peak of the observed LF, with an uncertainty ascertained through bootstrap resampling. Importantly, this procedure allows for nonGaussian HB magnitude distributions that are particularly relevant in the near-IR, where the HB may only be truly horizontal at nearsolar metallicities. The uncertainty of $\Delta m_{\mathrm{RGBB}}^{\mathrm{HB}}$ is calculated as the quadrature sum of the reported uncertainties on the HB and RGBB magnitude, and we conservatively assume an uncertainty of $0.1 \mathrm{mag}$ on the HB magnitude for clusters in Valenti et al. (2004b) and V10 without $\mathrm{HB}$ magnitude uncertainties.

In Fig. 14, we show linear fits of both $[\mathrm{Fe} / \mathrm{H}]$ and $[\mathrm{M} / \mathrm{H}]$ as a function of $\Delta m_{\mathrm{RGBB}}^{\mathrm{HB}}$ (in this case using uncertainties in both axes to weight the fits; cf. Cohen et al. 2015), with the resulting coefficients given in each panel of Fig. 14 and summarized in Table 7. As only the relatively metal-rich $([\mathrm{M} / \mathrm{H}] \gtrsim-1.1)$ clusters in our sample show a detectable peak in their LF resulting from the $\mathrm{HB}$, the $\Delta m_{\mathrm{RGBB}}^{\mathrm{HB}}$ diagnostic is only applicable at these higher metallicities, but, nevertheless, the standard deviation of the fit residuals is $\leq 0.1$ dex in all cases.

However, it should be somewhat surprising that the magnitude difference between the RGBB and the HB is well fit by a linear relation since current observational and theoretical evidence implies that the luminosity of neither the RGBB nor the HB is strictly a linear function of metallicity. On the observational side, Valenti et al. (2004b) and Cohen et al. (2015) used a quadratic relation to fit the near-IR bump luminosity versus metallicity, while from
Table 7. Linear coefficients for photometric metallicity indicators: $y=A+$ $B x$.

\begin{tabular}{lcccl}
\hline$x$ & $y$ & $A$ & $B$ & \multicolumn{1}{c}{$\mathrm{rms}$} \\
\hline slope $J K$ & {$[\mathrm{Fe} / \mathrm{H}]$} & $-3.69 \pm 0.06$ & $-30.85 \pm 0.80$ & 0.20 \\
$\Delta J_{\mathrm{RGBB}}^{\mathrm{HB}}$ & {$[\mathrm{Fe} / \mathrm{H}]$} & $-0.929 \pm 0.020$ & $-0.969 \pm 0.050$ & 0.079 \\
$\Delta H_{\mathrm{RGBB}}^{\mathrm{HB}}$ & {$[\mathrm{Fe} / \mathrm{H}]$} & $-0.811 \pm 0.034$ & $-0.990 \pm 0.080$ & 0.076 \\
$\Delta K_{\mathrm{RGBB}}^{\mathrm{HB}}$ & {$[\mathrm{Fe} / \mathrm{H}]$} & $-0.806 \pm 0.018$ & $-0.899 \pm 0.047$ & 0.081 \\
$\Delta J_{\mathrm{TRGB}}^{\mathrm{RGBB}}$ & {$[\mathrm{Fe} / \mathrm{H}]$} & $-5.61 \pm 0.27$ & $1.03 \pm 0.06$ & 0.15 \\
$\Delta H_{\mathrm{TRGB}}^{\mathrm{RGBB}}$ & {$[\mathrm{Fe} / \mathrm{H}]$} & $-5.82 \pm 0.40$ & $1.00 \pm 0.08$ & 0.16 \\
$\Delta K_{\mathrm{TRGB}}^{\mathrm{RGBB}}$ & {$[\mathrm{Fe} / \mathrm{H}]$} & $-5.25 \pm 0.22$ & $0.86 \pm 0.05$ & 0.11 \\
slope $J K^{\mathrm{HB}}$ & {$[\mathrm{M} / \mathrm{H}]$} & $-3.31 \pm 0.09$ & $-29.92 \pm 1.10$ & 0.20 \\
$\Delta J_{\mathrm{RGBB}}^{\mathrm{HB}}$ & {$[\mathrm{M} / \mathrm{H}]$} & $-0.636 \pm 0.034$ & $-0.834 \pm 0.077$ & 0.093 \\
$\Delta H_{\mathrm{RGBB}}^{\mathrm{HB}}$ & {$[\mathrm{M} / \mathrm{H}]$} & $-0.552 \pm 0.045$ & $-0.870 \pm 0.114$ & 0.090 \\
$\Delta K_{\mathrm{RGBB}}^{\mathrm{HB}}$ & {$[\mathrm{M} / \mathrm{H}]$} & $-0.538 \pm 0.031$ & $-0.769 \pm 0.070$ & 0.089 \\
$\Delta J_{\mathrm{TRGB}}^{\mathrm{RGBB}}$ & {$[\mathrm{M} / \mathrm{H}]$} & $-5.11 \pm 0.27$ & $0.97 \pm 0.06$ & 0.15 \\
$\Delta H_{\mathrm{TRGB}}^{\mathrm{RGBB}}$ & {$[\mathrm{M} / \mathrm{H}]$} & $-5.29 \pm 0.39$ & $0.95 \pm 0.08$ & 0.16 \\
$\Delta K_{\mathrm{TRGB}}^{\mathrm{RGBB}}$ & {$[\mathrm{M} / \mathrm{H}]$} & $-4.76 \pm 0.22$ & $0.81 \pm 0.05$ & 0.13 \\
\hline & & & & \\
\hline
\end{tabular}

a theoretical perspective, the models of Salaris \& Girardi (2002) suggest that the $K_{S}$ luminosity of the HB is a non-linear function of metallicity even at fixed age. Along similar lines, both the RGBB and HB magnitudes are predicted to depend on second, and likely third parameters in addition to metallicity. The Salaris \& Girardi (2002) models predict that the $K_{S}$ luminosity of the HB depends on age as well as metallicity (at a level of $\leq 0.05 \mathrm{mag} \mathrm{Gyr}^{-1}$ for typical GGC ages), although deep IR photometry of optically well-studied clusters is needed to confirm this predictions. Regarding the RGBB, Salaris et al. (2007) point out that at least at the metallicity of 47 Tuc, $\alpha$-enhanced BaSTI models (Pietrinferni et al. 2006) predict that the near-IR luminosity of the RGBB is affected by changes in $[\alpha / \mathrm{Fe}]$ (at a level of $\Delta K_{S}=0.06 \mathrm{mag}$ for $\Delta[\alpha / \mathrm{Fe}]=0.4$ ), and to a somewhat lesser extent by age (although in this case, models predict that the RGBB and HB change in the same direction). The data that we employ are insufficient to confirm or deny whether a 


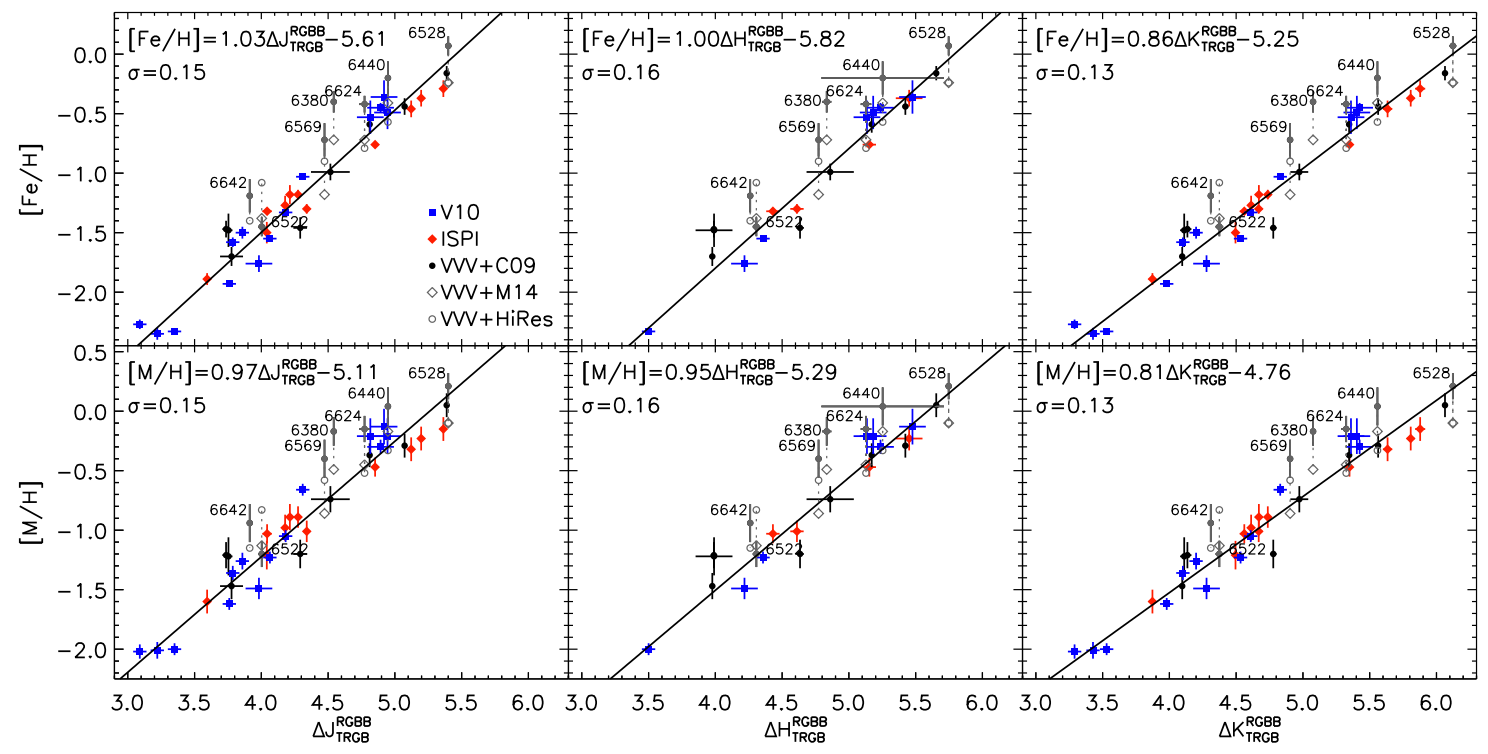

Figure 15. Linear fits in all three $J H K_{S}$ filters to metallicity as a function of the magnitude difference between the RGBB and the TRGB. Note the difference in axis scales as compared to Fig. 14. Symbols are as in Fig. 13, but the horizontal error bars represent only uncertainties in the RGBB magnitude and are not used in the fits.

higher order fit to the $\Delta m_{\mathrm{RGBB}}^{\mathrm{HB}}-$ metallicity relation is appropriate or not, and more secure ages and spectroscopic abundances for bulge GGCs could improve the situation. However, the current typical uncertainties of $\sim 0.1$ dex on global metallicity and $\sim 0.05$ mag on $\Delta m_{\mathrm{RGBB}}^{\mathrm{HB}}$ would not permit the detection of a subtle non-linearity in the relation, and indeed the relations between metallicity and near-IR RGBB and HB magnitude from Cohen et al. (2015), along with their uncertainties, imply that in the higher metallicity regime explored here $([\mathrm{M} / \mathrm{H}]<1)$, a relation between metallicity and $\Delta m_{\mathrm{RGBB}}^{\mathrm{HB}}$ is expected to be linear at the $\sim 0.1$ dex level.

\subsection{RGB bump-tip Magnitude Difference $\left(\Delta m_{\mathrm{TRGB}}^{\mathrm{RGBB}}\right)$}

The parameter $\Delta m_{\mathrm{TRGB}}^{\mathrm{RGBB}}$ is another potential metallicity indicator that we explore in the IR for the first time. Additionally, given that the RGBB magnitude can generally be measured with much greater precision than the TRGB magnitude (see Section 3.2, Appendix A and Table 3), we also explore the precision of the $\Delta m_{\mathrm{TRGB}}^{\mathrm{RGBB}}$ versus metallicity relations as a vehicle to empirically quantify the uncertainty of the TRGB magnitude. Relations between $\Delta m_{\mathrm{TRGB}}^{\mathrm{RGB}}$ and metallicity are shown in Fig. 15, and as the uncertainty in the TRGB location dominates the uncertainty in that of the RGBB, we weight our least-squares fit only by the (y-axis) uncertainty in metallicity. If we invert the best-fitting relations and calculate the rms residuals of a fit with respect to $\Delta m_{\mathrm{TRGB}}^{\mathrm{RGBB}}$ rather than metallicity, we obtain values of $0.14<\sigma \Delta m_{\mathrm{TRGB}}^{\mathrm{RGBB}}<0.17 \mathrm{mag}$. As the uncertainty in the RGBB location generally contributes negligibly to this quantity (the VVV and ISPI samples have median uncertainties of $<0.03$ mag on $\left.m_{\mathrm{RGBB}}\right)$, it would appear that the median uncertainty on the location of the TRGB is $<0.2 \mathrm{mag}$ in the near-IR, somewhat smaller than the typical values obtained by Valenti et al. (2004b, see their table 3 ) based on the prescription employed by Ferraro et al. (1999): The median of their reported TRGB uncertainties is $(0.25,0.23,0.26)$ mag in $\left(J, H, K_{S}\right)$. However, the discussion in Appendix A is presented to highlight the complexity of attempting to measure the TRGB location in GGCs, and when viewed on a case-by-case basis, ambiguities in the TRGB location are often larger than a naive extrapolation of the residuals of our linear fits in Fig. 15 would imply.

For convenience, the coefficients of the linear fits shown in Figs 13-15, along with their uncertainties and the rms residuals of the fits, are summarized in Table 7.

\section{DISCUSSION}

Our results shown in Figs 13-15 suggest that the three photometric metallicity indicators slope ${ }_{J K}, \Delta m_{\mathrm{RGBB}}^{\mathrm{HB}}$ and $\Delta m_{\mathrm{TRGB}}^{\mathrm{RGBB}}$ each have their respective advantages in different metallicity regimes. At relatively high $([\mathrm{M} / \mathrm{H}] \gtrsim-1)$ metallicities, $\Delta m_{\mathrm{RGBB}}^{\mathrm{HB}}$ yields the best overall precision with an rms deviation of $<0.1$ dex from our linear fit in all three $J H K_{S}$ filters, although both slope ${ }_{J K}$ and $\Delta m_{\mathrm{TRGB}}^{\mathrm{RGB}}$ do nearly as well, with rms deviations of $\sim 0.15$ dex. However, moving to lower metallicities, $\Delta m_{\mathrm{RGBB}}^{\mathrm{HB}}$ becomes difficult to apply for two reasons. First, clusters that are more metal-rich tend to have HB magnitudes that can be more reliably measured in the near-IR due to the increased horizontality of the HB in near-IR CMDs. For example, a peak in the cluster LF corresponding to the HB could not be reliably detected for clusters with $[\mathrm{Fe} / \mathrm{H}](\mathrm{C} 09)<-1.2$, similar to the results of Cohen et al. (2015). Secondly, the RGB bump becomes less prominent with decreasing metallicity (Nataf et al. 2013), also hindering the use of $\Delta m_{\mathrm{TRGB}}^{\mathrm{RGBB}}$. Therefore, despite its somewhat poorer rms deviation of $\sim 0.2 \mathrm{dex}$, slope ${ }_{J K}$ may be the best option at lower metallicities, particularly for relatively sparse stellar populations where either the RGBB and/or the TRGB location cannot be reliably measured.

We can apply the calibrations listed in Table 7 to obtain purely photometric metallicity estimates for our target clusters. For each cluster, there are a total of seven calibrations available to calculate $[\mathrm{Fe} / \mathrm{H}]$ or $[\mathrm{M} / \mathrm{H}]$, and we exclude those for which data are not available in individual cases (i.e. the $\mathrm{HB}$ magnitudes for metalpoor clusters). The resulting mean photometric $[\mathrm{Fe} / \mathrm{H}]$ and $[\mathrm{M} / \mathrm{H}]$, weighted by the inverse quadrature sum of the observational uncertainty and the calibration rms, are given in Table 8 along with the number of relations from Table 7 available. These photometric metallicity estimates are compared in Fig. 16 with $[\mathrm{Fe} / \mathrm{H}]$ values 
Table 8. Photometric metallicity estimates for target clusters.

\begin{tabular}{lccc}
\hline Cluster & {$[\mathrm{Fe} / \mathrm{H}]$} & {$[\mathrm{M} / \mathrm{H}]$} & $N($ relations $)$ \\
\hline NGC6380 & $-0.81 \pm 0.05$ & $-0.55 \pm 0.05$ & 7 \\
NGC6401 & $-1.34 \pm 0.21$ & $-1.03 \pm 0.21$ & 1 \\
NGC6440 & $-0.46 \pm 0.06$ & $-0.23 \pm 0.06$ & 7 \\
NGC6441 & $-0.44 \pm 0.05$ & $-0.21 \pm 0.05$ & 7 \\
NGC6453 & $-1.80 \pm 0.12$ & $-1.49 \pm 0.12$ & 4 \\
NGC6522 & $-1.38 \pm 0.11$ & $-1.10 \pm 0.11$ & 4 \\
NGC6528 & $-0.08 \pm 0.05$ & $0.10 \pm 0.05$ & 7 \\
NGC6544 & $-1.69 \pm 0.12$ & $-1.39 \pm 0.12$ & 4 \\
NGC6553 & $-0.23 \pm 0.05$ & $-0.03 \pm 0.05$ & 7 \\
NGC6558 & $-1.18 \pm 0.22$ & $-0.87 \pm 0.22$ & 1 \\
NGC6569 & $-1.00 \pm 0.05$ & $-0.71 \pm 0.05$ & 7 \\
NGC6624 & $-0.58 \pm 0.05$ & $-0.34 \pm 0.05$ & 7 \\
M28 & $-1.17 \pm 0.11$ & $-0.90 \pm 0.11$ & 4 \\
M69 & $-0.60 \pm 0.05$ & $-0.37 \pm 0.05$ & 7 \\
NGC6638 & $-1.09 \pm 0.07$ & $-0.79 \pm 0.07$ & 7 \\
NGC6642 & $-1.55 \pm 0.05$ & $-1.20 \pm 0.05$ & 7 \\
M22 & $-1.71 \pm 0.11$ & $-1.41 \pm 0.11$ & 4 \\
\hline
\end{tabular}

from Dias et al. (2016), M14, the HiRes spectroscopic values in Table 5, and the $\mathrm{H} 10$ catalogue.

In general, our linear fits in Figs 13-15 favour recent spectroscopic metallicities over those listed in the compilation of C09 for metal-rich clusters (NGC 6380, 6440, 6528, 6569 and to a lesser extent NGC 6624). For the six target clusters with $[\mathrm{Fe} / \mathrm{H}] \lesssim-0.7$, the agreement with the H10 catalogue is particularly good, with a mean offset of $-0.03 \pm 0.02$ dex. For NGC 6528, arguably the most metal-rich GGC, our calibrations give $[\mathrm{Fe} / \mathrm{H}]$ between the lower values reported by Zoccali et al. (2004), Origlia et al. (2005) and Sobeck et al. (2006) and the supersolar value of Carretta et al. (2001), in good agreement with the low-resolution spectra of Dias et al. (2015).

In a global sense, our photometric metallicity calibrations agree best with the Ca II triplet values of M14 compared to the other sets of literature metallicity values. For example, our photometric metallicities imply a decrease in $[\mathrm{Fe} / \mathrm{H}]$ of $\sim 0.3-0.4$ dex for NGC 6380 and NGC 6569 compared to their C09 values. For NGC 6380, this is also in agreement with the H10 catalogue, while NGC 6569 is a significant outlier in the $\mathrm{Ca}$ II triplet calibration of M14, who found $[\mathrm{Fe} / \mathrm{H}]=-1.18 \pm 0.11$, in better $(\sim 2 \sigma)$ accord with our photometric metallicities than any spectroscopic results. For NGC 6401 and NGC 6558 , our photometric values are $\sim 0.2$ dex lower than those measured from spectroscopy. However, because these clusters lack a detectable RGBB or red $\mathrm{HB}$, our photometric metallicity estimate is based only on the RGB slope, so the uncertainties are relatively large. Furthermore, since these clusters are relatively sparse and projected on the Galactic bulge, their TRGB magnitudes remain uncertain at the $\gtrsim 0.3 \mathrm{mag}$ level (see Appendix A). This implies yet a larger corresponding uncertainty of the RGB slope and hence the photometric metallicity should our chosen TRGB candidate be proven incorrect. For the remainder of metal-intermediate blue $\mathrm{HB}$ clusters in the VVV sample, we also find metallicities $>0.2-0.3$ dex lower than those of C09. For NGC 6544, the C09 value is supported by M14, although the discrepancy between their result and ours is only marginally significant in light of the large uncertainties. Meanwhile, our photometric $[\mathrm{Fe} / \mathrm{H}]$ value for NGC 6453 rests fairly heavily on the assumed TRGB magnitude, and, in fact, our photometric $[\mathrm{M} / \mathrm{H}]$ value is in good agreement with Dias et al. (2016) if this cluster has a relatively low level of $\alpha$-enhancement as suggested by their fits to synthetic spectra. Lastly, for NGC 6642, the value given by our photometric calibrations agrees with Minniti (1995) to within the uncertainties, and a value as low as $[\mathrm{Fe} / \mathrm{H}]=$ -1.8 was suggested by Balbinot et al. (2009) based on isochrone fitting to space-based optical photometry.

Our results underscore the need for high-resolution multi-object spectroscopy of poorly studied bulge GGCs. The tendency of the C09 compilation to overestimate the $[\mathrm{Fe} / \mathrm{H}]$ of metal-rich bulge clusters could be simply an artefact of high field star densities in the original integrated light studies compiled by $\mathrm{C} 09$ and/or the use of a supersolar metallicity for NGC 6528 to convert previous metallicity scales to their UVES scale. In either case, the GGC metallicity scale at high metallicities remains poorly constrained, and detailed spectroscopic analyses of large samples of cluster stars (for example, to assess contamination by AGB members) are crucial for accurate and self-consistent determinations of $[\mathrm{Fe} / \mathrm{H}]$ as well as $[\alpha / \mathrm{Fe}]$. This would be a valuable step towards testing GGC evolutionary

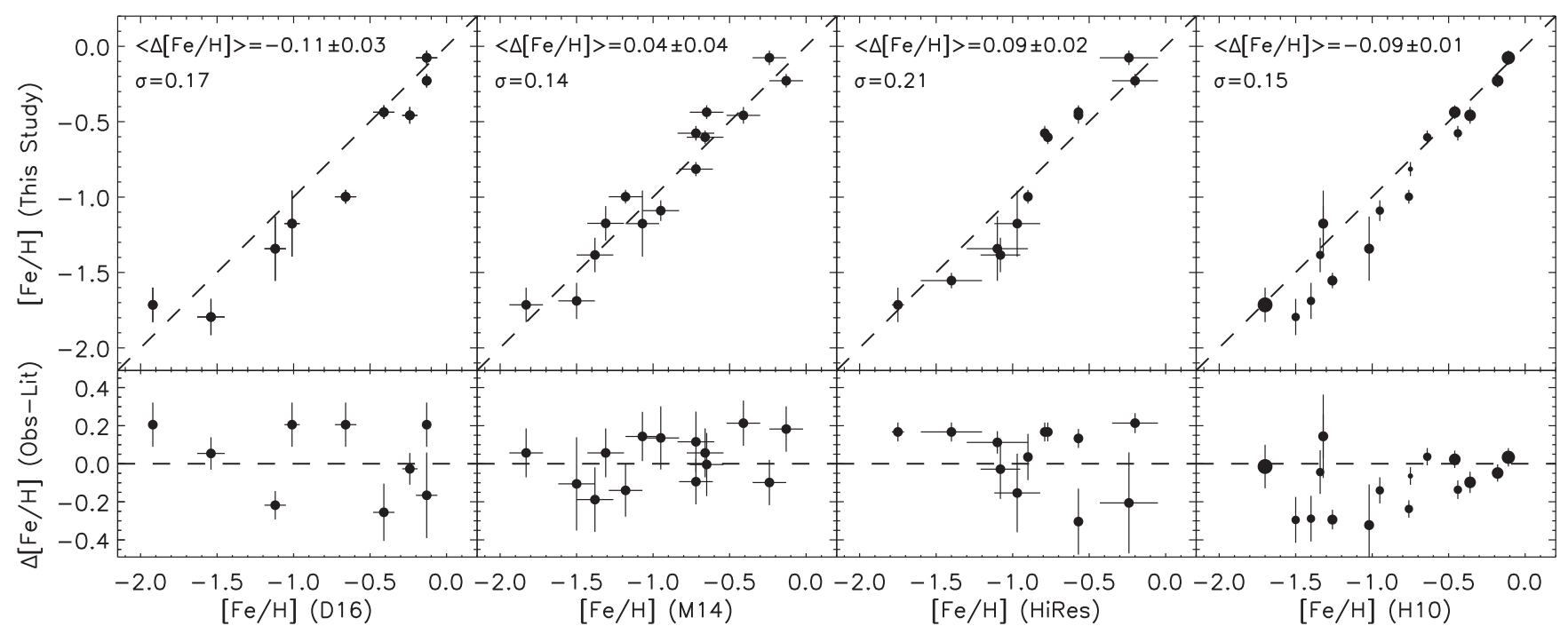

Figure 16. Comparison between our photometric $[\mathrm{Fe} / \mathrm{H}]$ estimates given in Table 8 and $[\mathrm{Fe} / \mathrm{H}]$ values from (left to right) Dias et al. (2016), M14, the HiRes values in Table 5 and the $\mathrm{H} 10$ catalogue. In each plot, the dashed line indicates equality. Because $\mathrm{H} 10$ give weights rather than formal uncertainties on their $[\mathrm{Fe} / \mathrm{H}]$ values, the size of the plotting symbol is proportional to the weight given to the $[\mathrm{Fe} / \mathrm{H}]$ value for each cluster in the rightmost panel by $\mathrm{H} 10$. 
models at near-solar metallicities and improving our relations to allow a deeper understanding of distant, composite and/or heavily extincted stellar populations.

\section{ACKNOWLEDGEMENTS}

It is a pleasure to thank Jim Emerson for discussions regarding the calibration of VISTA photometry to the 2MASS photometric system, and the anonymous referee for their insightful comments, that enhanced the quality and presentation of this study. REC is grateful for financial support from Fondo GEMINI-CONICYT 32140007 , and FM is thankful for financial support from FONDECYT for project 3140177. DG, FM and REC also acknowledge financial support from the Chilean BASAL Centro de Excelencia en Astrofisica y Technologias Afines (CATA) grant PFB-06/2007. CMB acknowledges financial support from FONDECYT through regular project 1150060 . This publication makes use of data products from the Two Micron All Sky Survey (2MASS), that is a joint project of the University of Massachusetts and the Infrared Processing and Analysis Center/California Institute of Technology, funded by the National Aeronautics and Space Administration and the National Science Foundation. This publication is based largely on observations collected at the European Organisation for Astronomical Research in the Southern hemisphere, Chile, under ESO program 179.B-2002 (VVV survey).

\section{REFERENCES}

Abbas M. A. et al., 2015, AJ, 149, 40

Alonso Garcia J., Mateo M., Sen B., Banerjee M., Catelan M., Minniti D., von Braun K., 2012, AJ, 143, 70

Alves D. R., Sarajedini A., 1999, ApJ, 511, 225

Alves-Brito A. et al., 2006, A\&A, 460, 269

Alves-Brito A., Yong D., Melendez J., Vasquez S., Karakas A. I., 2012, A\&A, 540, 3

Ammons S. M., Robinson S. E., Strader J., Laughlin G., Fischer D., Wolf A., 2006, ApJ, 638, 1004

Arellano Ferro A., Bramich D. M., Giridhar S., Figuera Jaimes R., Kains N., Kuppuswamy K., 2013, Acta. Astron., 63, 429

Arellano Ferro A., Bramich D. M., Giridhar S., Luna A., Muneer S., 2015, Inf. Bull. Var. Stars, 6137, 1

Balbinot E., Santiago B. X., Bica E., Bonatto C., 2009, MNRAS, 396, 1596

Barbuy B., Zoccali M., Ortolani S., Minniti D., Hill V., Renzini A., Bica E., Gómez A., 2007, AJ, 134, 1613

Barbuy B. et al., 2014, A\&A, 570, A76

Bedding T. R. et al., 2010, ApJ, 713, L176

Bessell M. S., Brett J. M., 1988, PASP, 100, 1134

Bonatto C., Bica E., 2007, MNRAS, 377, 1301

Bonatto C., Bica E., 2010, A\&A, 516, A81

Calamida A. et al., 2014, A\&A, 565, A8

Caloi V., D’Antona F., 2005, A\&A, 435, 987

Carretta E., 2006, AJ, 131, 1766

Carretta E., Cohen J. G., Gratton R. G., Behr B. B., 2001, AJ, 122, 1469

Carretta E., Bragaglia A., Gratton R., D’Orazi V., Lucatello S., 2009, A\&A, 508, 695 (C09)

Carretta E., Bragaglia A., Gratton R. G., Recio-Blanco A., Lucatello S., D’Orazi V., Cassisi S., 2010, A\&A, 516, 55

Casagrande L., VandenBerg D. A., 2014, MNRAS, 444, 392

Cassisi S., Salaris M., 1997, MNRAS, 285, 593

Chené A.-N. et al., 2012, A\&A, 545, A54

Cho D.-H., Lee S.-G., 2002, AJ, 124, 977

Chun S.-H. et al., 2010, A\&A, 518, 15

Clement C. M. et al., 2001, AJ, 122, 2587

Cohen R. E., Mauro F., Geisler D., Moni Bidin C., Dotter A., Bonatto C., 2014, AJ, 148, 18
Cohen R. E., Hempel M., Mauro F., Geisler D., Alsono Garcia J., Kinemuchi K., 2015, AJ, 150, 176

Conn A. R. et al., 2011, ApJ, 740, 69

Dalcanton J. J. et al., 2012, ApJS, 198, 6

Dalessandro E., Salaris M., Ferraro F. R., Mucciarelli A., Cassisi S., 2013, MNRAS, 430, 459

Di Cecco A. et al., 2010, ApJ, 712, 527

Dias W. S., Monteiro H., Caetano T. C., Léépine J. R. D., Assafin M., Oliveira A. F., 2014, A\&A, 564, A79

Dias B., Barbuy B., Saviane I., Held E. V., Da Costa G. S., Ortolani S., Vasquez S., Gullieuszik M., Katz D., 2015, A\&A, 573, A13

Dias B., Barbuy B., Saviane I., Held E. V., Da Costa G. S., Ortolani S., Gullieuszik M., Vàsquez S., 2016, A\&A, 590, A9

Dotter A., Chaboyer B., Jevremovic D., Kostov V., Baron E., Ferguson J. W., 2008, ApJS, 178, 89

Emerson J., McPherson A., Sutherland W., 2006, The Messenger, 126, 41

Feltzing S., Johnson R. A., 2002, A\&A, 385, 67

Ferraro F. R., Messineo M., Fusi Pecci F., de Palo M. A., Straniero O., Chieffi A., Limongi M., 1999, AJ, 118, 1738

Ferraro F. R., Montegriffo P., Origlia L., Fusi Pecci F., 2000, AJ, 119, 1282

Fusi Pecci F., Ferraro F. R., Crocker D. A., Rood R. T., Buonanno R., 1990, A\&A, 238, 95

Gonzalez O. A., Rejkuba M., Zoccali M., Valenti E., Minniti D., Schultheis M., Tobar R., Chen B., 2012, A\&A, 543, 13

Gorski M. et al., 2016, ApJ, 151, 167

Grocholski A. J., Sarajedini A., 2002, AJ, 123, 1603

Gullieuszik M., Held E. V., Rizzi L., Saviane I., Momany Y., Ortolani S. 2007, A\&A, 467, 1025

Harris W. E., 1996, AJ, 112, 1487 (H10)

Hartwick F. D. A., Barlow D. J., Hesser J. E., 1981, AJ, 86, 1044

Hendricks B., Stetson P. B., VandenBerg D. A., Dall'Ora M., 2012, AJ, 144, 25

Hughes J., Wallerstein G., Covarrubias R., Hays N., 2007, AJ, 134, 229

Iben I., 1968, Nature, 220, 143

Ivanov V. D., Borissova J., 2002, A\&A, 390, 937

Johnson C. I., Pilachowski C. A., 2012, ApJ, 754, L38

Kiss L. L., Bedding T. R., 2003, MNRAS, 343, L79

Kiss L. L., Bedding T. R., 2004, MNRAS, 347, L83

Kordopatis G. et al., 2013, AJ, 146, 134

Kuchinski L. E., Frogel J. A., Terndrup D. M., Persson S. E., 1995, AJ, 109, 1131

Layden A. C., Ritter L. A., Welch D. L., Webb T. M. A., 1999, AJ, 117, 1313

Layden A. C. et al., 2010, PASP, 122, 1000

Lebzelter T., Wood P. R., 2005, A\&A, 441, 1117

Lebzelter T., Wood P. R., 2011, A\&A, 529, 137

Lebzelter T., Wood P. R., Hinkle K. H., Joyce R. R., Fekel F. C., 2005, A\&A, 432, 207

Lee J. W., 2007, Rev. Mex. Astron. Astrofis., 28, 120

Lee M. G., Freedman W. L., Madore B. F., 1993, ApJ, 417, 553

Libralato M., Bellini A., Bedin L. R., Piotto G., Platais I., Kissler-Patig M., Milone A. P., 2014, A\&A, 563, A80

Libralato M. et al., 2015, MNRAS, 450, 1664

McDonald I., van Loon J. T., Dupree A. K., Boyer M. L., 2010, MNRAS, 405, 1711

McLaughlin D. E., van der Marel R. P., 2005, ApJS, 161, 304

Makarov D., Makarova L., Rizzi L., Tully R. B., Dolphin A. E., Sakai S., Shaya E. J., 2006, AJ, 132, 2729

Marino A. F. et al., 2011, A\&A, 532, 8

Marino A. et al., 2012, A\&A, 541, 15

Marino A. F., Milone A. P., Lind K., 2013, ApJ, 768, 27

Massari D. et al., 2012, ApJ, 755, L32

Matsunaga N., Fukushi H., Nakada Y., 2005, MNRAS, 364, 117

Matsunaga N., Fukushi H., Nakada Y., 2006, MNRAS, 370, 1979

Matsunaga N., Kawadu T., Nishiyama S., Nagayama T., Hatano H., Tamura M., Glass I. S., Nagata T.2009, MNRAS, 399, 1709

Mauro F., Moni Bidin C., Cohen R., Geisler D., Minniti D., Catelan M. Chenéé A.é-N., Villanova S., 2012, ApJ, 761, 29 
Mauro F. et al., 2013, Rev. Mex. Astron. Astrofis., 49, 189

Mauro F. et al., 2014, A\&A, 563, 76 (M14)

Méndez B., Davis M., Moustakas J., Newman J., Madore B. F., Freedman W. L., 2002, AJ, 124, 213

Mészáros S., Dupree A. K., Szalai T., 2009, AJ, 137, 4282

Milone A. P. et al., 2012, A\&A, 540, 16

Milone A. P. et al., 2014, ApJ, 785, 21

Minniti D., 1995, A\&A, 303, 468

Minniti D. et al., 2011, A\&A, 527, 81

Monaco L., Pancino E., Ferraro F. R., Bellazzini M., 2004, MNRAS, 349, 1278

Moni Bidin C. et al., 2011, A\&A, 535, 33

Origlia, L,.Montegriffo P., Ferraro F. R., Fusi Pecci F.1995, MNRAS, 276, 738

Mucciarelli A., Lapenna E., Massari D., Pancino E., Stetson P. B., Ferraro F. R., Lanzoni B., Lardo C., 2015, ApJ, 809, 128

Nataf D. M., Udalski A., Gould A. P., Pinsonneault M. H., 2011, ApJ, 730, 118

Nataf D. M., Gould A. P., Pinsonneault M. H., Udalski A., 2013, ApJ, 766, 77

Ness M., Asplund M., Casey A. R., 2014, MNRAS, 445, 2994

Origlia L., Rich R. M., Castro S., 2002, AJ, 123, 1559

Origlia L., Valenti E., Rich R. M., 2005, MNRAS, 356, 1276

Origlia L., Valenti E., Rich R. M., 2008, MNRAS, 388, 1419

Peterson R. C., Cudworth K. M., 1994, ApJ, 420, 612

Pietrinferni A., Cassisi S., Salaris M., Castelli F., 2006, ApJ, 642, 797

Piotto G. et al., 2002, A\&A, 391, 945

Piotto G. et al., 2015, AJ, 149, 91

Prieto G., Catelan M., Contraras Ramos R., Pritzl B. J., Smith H. A., Alonso Garcia J., 2012, A\&A, 543, 148

Rees R. F., Cudworth K. M., 1991, AJ, 102, 152

Riello M. et al., 2003, A\&A, 410, 553

Rutledge G. A., Hesser J. E., Stetson P. B., 1997, PASP, 109, 907

Sahay A., Lebzelter T., Wood P. R., 2014, Publ. Astron. Soc. Austron., 31, 12

Saito R. et al., 2010, The Messenger, 141, 24

Saito R. K. et al., 2012, A\&A, 537, 107

Salaris M., Girardi L., 2002, MNRAS, 337, 332

Salaris M., Chieffi A., Straniero O., 1993, ApJ, 414, 580

Salaris M., Held E. V., Ortolani S., Gullieuszik M., Momany Y., 2007, A\&A, 476,243

Samus N. N., Kazarovets E. V., Pastukhova E. N., Tsvetkova T. M., Durlevich O. V., 2009, PASP, 121, 1378

Sarajedini A. et al., 2007, AJ, 133, 1658

Saviane I., da Costa G. S., Held E. V., Sommariva V., Gullieuszik M., Barbuy B., Ortolani S., 2012, A\&A, 540, 27

Schultheis M. et al., 2014, AJ, 148, 24

Skottfelt J. et al., 2015, A\&A, 573, A103

Skrutskie M. F. et al., 2006, AJ, 131, 1163

Sloan G. C. et al., 2010, ApJ, 719, 1274

Sobeck J. S., Ivans I. I., Simmerer J. A., Sneden C., Hoeflich P., Fulbright J. P., Kraft R. P., 2006, AJ, 131, 2949

Soszynski I., Udalski A., Kubiak M., Szymanski M., Pietrzynski G., Zebrun K., Szewczyk O., Wyrzykowski L., 2004, Acta. Astron., 54,129

Soszyński I. et al., 2013, Acta. Astron., 63, 21

Stetson P. B., 1987, PASP, 99, 191

Stetson P. B., 1994, PASP, 106, 250

Thomas H.-C., 1967, Z. Astrophys., 67, 420

Trager S. C., King I. R., Djorgovski S., 1995, AJ, 109, 218

Udalski A. et al., 2002, Acta. Astron., 52, 217

Valenti E., Ferraro F. R., Origlia L., 2004a, MNRAS, 351, 1204

Valenti E., Ferraro F. R., Origlia L., 2004b, MNRAS, 354, 815

Valenti E., Ferraro F. R., Origlia L., 2010, MNRAS, 402, 1729 (V10)

Valenti E., Origlia L., Rich R. M., 2011, MNRAS, 414, 2690

Valenti E., Origlia L., Mucciarelli A., Rich R. M., 2015, A\&A, 574, 80

VandenBerg D. A., Brogaard K., Leaman R., Casagrande L., 2013, ApJ, 775,134
VandenBerg D. A., Bergbusch P. A., Ferguson J. W., Edvardsson B., 2014, ApJ, 794, 72

Wray J. J., Eyer L., Paczyński B., 2004, MNRAS, 349, 1059

Zloczewski K., Kaluzny J., Rozyczka M., Krzeminski W., Mazur B., 2012, Acta. Astron., 62, 357

Zoccali M., Cassisi S., Piotto G., Bono G., Salaris M., 1999, ApJ, 518, L49

Zoccali M. et al., 2004, A\&A, 423, 507

Zoccali M., Hill V., Lecureur A., Barbuy B., Renzini A., Minniti D., Góómez A., Ortolani S., 2008, A\&A, 486, 177

\section{SUPPORTING INFORMATION}

Additional Supporting Information may be found in the online version of this article:

cmds_lfs.pdf

(http://www.mnras.oxfordjournals.org/lookup/suppl/doi:10.1093/ mnras/stw2435/-/DC1).

Please note: Oxford University Press is not responsible for the content or functionality of any supporting materials supplied by the authors. Any queries (other than missing material) should be directed to the corresponding author for the article.

\section{APPENDIX A: DETAILS OF THE TRGB IDENTIFICATION}

Here we give the details concerning the choice of the brightest RGB member star in each cluster. Care has been taken to use published proper motion, photometric variability, radial velocity and/or chemical abundance studies as well as additional photometry from the literature where available in order to assess the likelihood that a CMD-selected TRGB candidate is a cluster RGB member. As this still leaves the choice of TRGB star ambiguous in some cases, radial location in the cluster is also employed to judge the membership of TRGB candidates, so in many cases, we refer to the radial distance from the centre of the cluster as a fraction of the core radius $\left(R_{\mathrm{c}}\right)$ or half-light radius $\left(R_{\mathrm{hl}}\right)$ from the $\mathrm{H} 10$ catalogue. ${ }^{13}$ Clusters are listed by the source of their photometry, with the VVV clusters from the present study first, followed by those from Cohen et al. (2015), and finally those from Valenti et al. (2004b) and V10 absent from the two more recent studies. The TRGB candidate that we have chosen in each cluster is given in Table A1, along with its position, photometry, 2MASS ID, and the source of the given position and photometry. Although all TRGB candidates could be reliably matched to 2MASS counterparts, where possible, we have employed the photometry from the given source (corrected for differential reddening in the case of the VVV clusters) in place of 2MASS due to the improved spatial resolution of the source catalogue over the 2MASS PSC, as well as photometric quality flags in 2MASS warning of low-quality photometry. Conversely, in some casesm available near-IR catalogues saturate below the TRGB, requiring the use of photometry from 2MASS.

NGC 6380: the selected tip star has Xflg $=2$ (signifying that it is within an extended source) in 2MASS. There are several slightly brighter candidate tip stars present in 2MASS or only in the V10 catalogue, but all of these have $J-H$ and/or $J-K_{S}$ colours somewhat ( $\sim 0.1 \mathrm{mag}$ ) discrepant from the observed cluster RGB. Moving faintward, if our chosen TRGB candidate is not a true RGB star,

\footnotetext{
13 The structural parameters from H10 may not be reliable in some cases, as discussed by McLaughlin \& van der Marel (2005).
} 
Table A1. Selected TRGB Candidates.

\begin{tabular}{|c|c|c|c|c|c|c|c|}
\hline Cluster & RA(J2000) & Dec.(J2000) & $J(\mathrm{TRGB})$ & $H(\mathrm{TRGB})$ & $K_{S}(\mathrm{TRGB})$ & 2MASS ID & Source \\
\hline NGC6380 & 263.600525 & -39.064743 & 10.526 & 9.331 & 8.770 & $\mathrm{~J} 17342412-3903530$ & 2MASS \\
\hline NGC6401 & 264.650427 & -23.907622 & 10.436 & 9.348 & 9.040 & J17383610-2354275 & V10 \\
\hline NGC6440 & 267.226342 & -20.367685 & 10.214 & 8.977 & 8.459 & J14485434-2022034 & V10 \\
\hline NGC6441 & 267.558868 & -37.065826 & 10.532 & 9.470 & 9.188 & J17501414-3703569 & V10 \\
\hline NGC6453 & 267.715425 & -34.610149 & 10.622 & 9.673 & 9.402 & J17505170-3436365 & V10 \\
\hline NGC6522 & 270.871602 & -30.046986 & 9.863 & 8.882 & 8.649 & J18032918-3002491 & V10 \\
\hline NGC6528 & 271.182471 & -30.047783 & 9.338 & 8.265 & 7.739 & $\mathrm{~J} 18044378-3002523$ & V10 \\
\hline NGC6544 & 271.846548 & -24.976402 & 7.715 & 6.713 & 6.364 & $\mathrm{~J} 18072317-2458350$ & 2MASS \\
\hline NGC6553 & 272.325166 & -25.911547 & 8.499 & 7.415 & 6.812 & J18091804-2254415 & V10 \\
\hline NGC6558 & 272.573617 & -31.760925 & 10.079 & 9.065 & 8.822 & J18101766-3145393 & 2MASS \\
\hline NGC6569 & 273.410993 & -31.835494 & 10.471 & 9.479 & 9.192 & J18133863-3150077 & V10 \\
\hline NGC6624 & 275.943807 & -30.317759 & 9.536 & 8.534 & 8.234 & J18234651-3019039 & 2MASS \\
\hline M28 & 276.174360 & -24.884188 & 8.825 & 7.852 & 7.548 & $\mathrm{~J} 18244184-2453030$ & 2MASS \\
\hline M69 & 275.885411 & -32.293312 & 9.574 & 8.637 & 8.358 & $\mathrm{~J} 1831249-3217359$ & 2MASS \\
\hline NGC6638 & 277.732604 & -25.500245 & 9.936 & 8.946 & 8.682 & J18305581-2530007 & V10 \\
\hline NGC6642 & 277.970396 & -23.476840 & 10.023 & 9.031 & 8.830 & J18315289-2328365 & V10 \\
\hline M22 & 277.062931 & -23.915266 & 7.737 & 6.966 & 6.722 & J18361510-2354549 & 2MASS \\
\hline NGC104 & 6.063092 & -72.076809 & 7.876 & 6.997 & 6.723 & J00241513-7204365 & 2MASS \\
\hline NGC0288 & 13.171358 & -26.557552 & 9.693 & 8.819 & 8.589 & J00524112-2633271 & 2MASS \\
\hline NGC362 & 15.821451 & -70.847116 & 9.414 & 8.699 & 8.467 & J01031723-7050496 & 2MASS \\
\hline NGC1261 & 48.065417 & -55.211288 & 10.819 & 10.025 & 9.808 & J03121569-5512406 & ISPI \\
\hline NGC1851 & 78.531782 & -40.040782 & 10.210 & 9.320 & 9.138 & J05140762-4002267 & ISPI \\
\hline NGC2808 & 137.987816 & -64.858240 & 9.978 & 9.096 & 8.793 & J09115707-6451296 & ISPI \\
\hline NGC4833 & 194.955866 & -70.904366 & 9.323 & 8.493 & 8.260 & J12594940-7054157 & 2MASS \\
\hline NGC5927 & 231.992006 & -50.656418 & 9.222 & 8.233 & 7.882 & $\mathrm{~J} 15275807-5039230$ & 2MASS \\
\hline NGC6304 & 258.638476 & -29.430115 & 9.014 & 7.879 & 7.488 & J17143323-2925484 & 2MASS \\
\hline NGC6496 & 269.737148 & -44.264393 & 9.661 & 8.709 & 8.381 & J17585691-4415517 & 2MASS \\
\hline NGC6584 & 274.551755 & -52.170807 & 10.583 & 9.727 & 9.504 & J18181242-5210149 & 2MASS \\
\hline NGC7099 & 325.089558 & -23.164424 & 9.394 & 8.873 & 8.627 & J21402149-2309518 & ISPI \\
\hline NGC5272 & 205.562545 & 28.390408 & 9.842 & 9.198 & 8.900 & $\mathrm{~J} 13421508+2823256$ & V04 \\
\hline NGC5904 & 229.650179 & 2.110380 & 9.068 & 8.210 & 8.041 & $\mathrm{~J} 15183604+0206373$ & 2MASS \\
\hline NGC6205 & 250.424819 & 36.447708 & 9.264 & 8.493 & 8.299 & $\mathrm{~J} 16414196+3626518$ & V04 \\
\hline NGC6341 & 259.285217 & 43.137569 & 9.629 & 8.973 & 8.922 & $\mathrm{~J} 17170841+4308149$ & V04 \\
\hline NGC6342 & 260.305511 & -19.572372 & 9.705 & 8.668 & 8.346 & J17211332-1934205 & V10 \\
\hline NGC6752 & 287.783875 & -60.031040 & 7.836 & 6.993 & 6.717 & J19110813-6001517 & 2MASS \\
\hline NGC6273 & 255.655961 & -26.266617 & 9.668 & 8.832 & 8.572 & J17023743-2615599 & V10 \\
\hline NGC6316 & 259.148447 & -28.127740 & 10.281 & 9.174 & 8.832 & J17163562-2807398 & V10 \\
\hline NGC6355 & 260.994566 & -26.351975 & 10.191 & 9.300 & 8.918 & J17235869-2621071 & V10 \\
\hline NGC6388 & 264.037095 & -44.760095 & 10.287 & 9.233 & 8.905 & J17360890-4445363 & V10 \\
\hline NGC6539 & 271.212936 & -7.571320 & 10.084 & 8.915 & 8.470 & J18045110-0734166 & V10 \\
\hline
\end{tabular}

selection of the next several fainter candidates with colours compatible with RGB membership would affect the TRGB magnitudes by $\pm 0.1 \mathrm{mag}$ in each of the three filters.

NGC 6401: although V10 select 2MASS J17383033-2352537, this star may not be a member: after applying differential reddening corrections, it lies slightly $(\sim 0.05)$ blueward of the cluster sequence in $J-H$, and lies at $\sim 1.15 R_{\mathrm{hl}}$. Our selected TRGB candidate is a much more likely member based both on differential reddeningcorrected V10 photometry and distance of only $\sim 9 \operatorname{arcsec}\left(<R_{\mathrm{c}}\right)$ from the cluster centre. This represents a faintward revision of $0.2-$ $0.3 \mathrm{mag}$ in the TRGB of this cluster from the value reported by V10, and we note that the RGB of NGC 6401 is relatively sparse, and Chun et al. (2010) chose to refrain from reporting a TRGB magnitude. However, if the uncertain RGBB magnitude we report is correct, then photometric as well as spectroscopic metallicity estimates for this cluster argue for a significantly brighter $(\gtrsim 0.5 \mathrm{mag})$ TRGB, that would also likely move the measured RGB slope value into better accord with clusters at similar metallicities.

NGC 6440: the selected TRGB candidate is the brightest with $J-K_{S}$ colour consistent with the observed cluster fiducial sequence, but is not detected in the $H$ band in the V10 catalogue. This star is a $2 \sigma(0.389 \mathrm{arcsec})$ positional match with 2MASS J174854342022034, that gives a $J-H$ colour consistent with this star being an RGB member, although 2MASS reports Xflg $=2$.

NGC 6441: we discard the several brightest CMD-selected candidates (variables V1, V131, V134, OGLE-BLG-LPV-060919) due to their long periods and large pulsational amplitudes. Our chosen candidate is V23, for which Layden et al. (1999) find evidence of long-term variability but are unable to further constrain pulsational properties. Furthermore, mean magnitudes from their optical photometry place this star on the cluster RGB. However, if this candidate should turn out to be an AGB star, the next best candidate is 2MASS J17501619-3702476, for which the differential reddening-corrected V10 catalogue implies a faintward TRGB shift of $<0.08 \mathrm{mag}$ in all three $J H K_{S}$ filters.

NGC 6453: the brightest TRGB candidate, also selected by V10 has a 94 per cent probability of membership to the open cluster M 9 (Dias et al. 2014), so we select the next candidate for which Dias et al. (2014) reports a 0 per cent membership probability to M 9. 
NGC 6522: the tip star selected by V10 is V5476 Sgr, identified as an OSARG (Soszyński et al. 2013), as is the brighter candidate V5471 Sgr. While their pulsational properties alone do not exclude the possibility of RGB status or cluster membership, in these cases as well as all brighter candidates (V5462 Sgr, V5468 Sgr, V5475 Sgr), a comparison with photometry of the surrounding field from Udalski et al. (2002) suggests that these are bulge, rather than cluster giants. Conversely, our selected TRGB candidate, V5466 Sgr, has mean $V$ and $I$ magnitudes more consistent with cluster membership. However, pending confirmation of membership for any of these variables, the TRGB magnitude may be subject to change by as much as $0.5 \mathrm{mag}$ in all three filters.

NGC 6528: the TRGB location for this cluster is also somewhat uncertain. The TRGB magnitudes we report correspond to differential reddening corrected photometry of 2MASS J18044378$3002523=$ OGLE-BLG-LPV-200787. Another brighter candidate, the OSARG OGLE-BLG-LPV-201338, cannot be excluded from membership based on pulsational properties or photometry, but has proper motions more consistent with bulge than cluster membership (Feltzing \& Johnson 2002). As in the similarly ambiguous cases above, should our choice of TRGB candidate be proven incorrect, the TRGB magnitudes would be affected by $>0.2$ mag in all three filters.

NGC 6544: as the cluster and Galactic bulge sequences are well separated in the CMD in this case, 2MASS J18072317-2458350 appears to be a fairly unambiguous choice. However, this star is $1.48 \operatorname{arcmin}\left(>4 R_{\mathrm{hl}}\right.$; Cohen et al. 2014) from the cluster centre, whereas there is another potential tip star (2MASS J180719372459558 ) that is only $0.17 \mathrm{mag}$ fainter in $K_{S}$ and is only $10.3 \mathrm{arcsec}$ $\left(<R_{\mathrm{c}}\right)$ from the cluster centre, although its 2MASS photometry may be somewhat unreliable given its value of $\mathrm{Cflg}=\mathrm{ddd}$ and $\mathrm{Xflg}=2$.

NGC 6553: we reject the brightest two CMD-selected TRGB candidates in our decontaminated catalogues, V4 and V5. V4 is a Mira variable, and both have colours inconsistent with the location of the cluster RGB (at the 0.1 mag level in $(J-H)$ ). We select the same TRGB candidate as V10, that lies well inside the cluster core radius.

NGC 6558: this is yet another case where the TRGB determination is particularly uncertain, and where Chun et al. (2010) did not report the TRGB magnitude from their near-IR photometry. The brightest potential candidate is V2, that may, in fact, be constant, and Samus et al. (2009) noted that this star may be affected by blending. The next two brightest candidates lack any discriminating membership information aside from our CMDs, and our chosen TRGB candidate has a 2 MASS position that is an $\sim 1.9$ arcsec match to star NGC 6558_8 from Dias et al. (2015). If a true match, this is a spectroscopically confirmed member (also see Zoccali et al. 2008), and lies much closer to the cluster centre $\left(0.2 \operatorname{arcmin} \sim 0.1 R_{\mathrm{hl}}\right)$ than the two brighter candidates ( $>1.3$ arcmin). However, if any of the brighter candidates is confirmed as an RGB member, the TRGB magnitude of this cluster could move brightward by $>0.3$ mag.

NGC 6569: we have excluded the long period variables V3 and V21. Although the former has no additional pulsational properties listed, 2MASS warns of low-quality photometry. We have also excluded 2MASS J18133939-3149209 in light of its blue colour in the V10 catalogue (this star also has low-quality photometry in 2MASS). However, if either this star or V3 are confirmed as RGB members, the TRGB magnitude would move brightward by $>0.2 \mathrm{mag}$.

NGC 6624: the only TRGB candidates brighter than our selection have colours from both 2MASS and the V10 catalogue inconsistent with the location of the cluster RGB. However, as our candidate lies $2.9 \operatorname{arcmin}\left(\sim 3.5 R_{\mathrm{hl}}\right)$ from the cluster centre, if either of these are revealed to be RGB members, the TRGB magnitude could move brightward by $>0.2 \mathrm{mag}$. Conversely, if none of these stars, including our selected TRGB candidate, are members, the TRGB magnitude would move faintward from the values we report by $>0.13 \mathrm{mag}$ in all three filters.

NGC 6626 (M28): the brightest three TRGB candidates in this cluster all lie to the blue side of the cluster RGB in $(J-H)$ colour. Of these, one is NV8, suggested by Prieto et al. (2012) to be a Type II Cepheid based on its period and light curve. Another one, V10, has a membership probability of 90 per cent (Rees \& Cudworth 1991) and an amplitude of $A_{V}=0.6$, and cannot be definitively excluded as an RGB star. Meanwhile, the brightest of the three in $K_{S}$ is not a known variable and is absent from the proper motion study of Rees \& Cudworth (1991), but sits $\sim 0.08$ mag blueward of the cluster RGB. As 2MASS indicates excellent photometric quality for this star, we exclude it as a candidate based on its blue colour. Our selected candidate is the brightest star with colours in excellent agreement with the observed cluster RGB, and is star 2-56 in Rees \& Cudworth (1991), who give a membership probability of 92 per cent. In addition, its $K_{S}$ magnitude of $7.548 \pm 0.024$ is in reasonable agreement with the RGB tip location of $K_{S}(\mathrm{TRGB})=$ $7.45 \pm 0.10$ reported by Chun et al. (2010). However, if any of the brighter, blue candidates are confirmed as RGB members, the TRGB magnitude would move brightward by $>0.1 \mathrm{mag}$ in all three filters.

NGC 6637 (M69): our CMD-selected TRGB candidate is nearly 4 $\operatorname{arcmin}\left(\sim 4.7 R_{\mathrm{hl}}\right)$ from the cluster centre and its membership status could therefore be considered uncertain. If, in fact, a non-member, the next brightest candidate after eliminating the large amplitude variables $\mathrm{V} 1$ and $\mathrm{V} 3$ is V7, that, if an RGB (not AGB) variable, would shift the TRGB magnitudes faintward by $>0.2$ mag.

NGC 6638: we adopt the same tip star as V10. Although this is variable V70 of Skottfelt et al. (2015), it is likely an RGB rather than AGB variable given the period and amplitude they report.

NGC 6642: this is another case of some ambiguity in determination of the TRGB magnitudes. We have selected the same TRGB star as V10, that is a likely member at $<R_{\mathrm{hl}}$. However, after eliminating the Mira variable V2578 Sgr, there is one significantly brighter (>0.4 mag in all three filters) candidate that survived our decontamination procedure, although it lies much further from the cluster centre $\left(\sim 2.4 R_{\mathrm{hl}}\right)$. Similarly, if our selected TRGB candidate turns out to be a non-member or AGB star, there is another candidate at $R<R_{\mathrm{c}}$ that is only 0.05 mag fainter in $K_{S}$ but $>0.3$ mag fainter in $J$ and $H$.

NGC 6656 (M22): in this case, there are two likely TRGB candidates with very similar photometry, both of which are confirmed members (Peterson \& Cudworth 1994). The brighter of the two is V9, that has been found to be periodic (with a period and amplitude compatible with RGB status) but more recently appeared to be in a quiescent phase (Clement et al. 2001; Sahay et al. 2014). The TRGB candidate we adopt is the same tip star selected by Monaco et al. (2004), that is $<0.05$ mag fainter than V9 in $J H K_{S}$ and is not a known variable. However, since this cluster is nearby and has a large core, we cannot exclude the possibility that shallow high spatial resolution imaging could reveal additional TRGB candidates, and we note that the 2MASS-PSC gives Xflg $=2$ for this star. Clusters from Cohen et al. (2015): NGC 104 (47 Tuc): the brightest candidate TRGB star is variable LW5 from Lebzelter \& Wood (2005), who claim a period of $74 \mathrm{~d}$ superimposed on a more long-term variation. Given this period and the relatively small amplitude shown in their Fig. 1, this could be an RGB star, although an AGB status cannot be excluded based on its variability. 
NGC 288: The tip star chosen both here and by Valenti et al. (2004b) is a known semi-regular variable with a $V$ amplitude of $A_{V}=0.22 \mathrm{mag}$ and a period of $103 \mathrm{~d}$ (Arellano Ferro et al. 2013). However, the next brightest non-variable star with a $\left(J-K_{S}\right)$ colour consistent with the location of the RGB is more than 0.5 (0.7) mag fainter in $J\left(K_{S}\right)$.

NGC 362: selection of the TRGB location in this cluster is complicated by the presence of several low-amplitude variables near the RGB tip. The brightest candidate is variable LW6 from Lebzelter \& Wood (2011) (= Sz56), that appears to lie slightly redward of the cluster RGB but is only $16 \operatorname{arcsec}\left(\sim 0.3 R_{\mathrm{hl}}\right)$ from the cluster centre. Given its short period ( $34 \mathrm{~d})$ and small amplitude $\left(A_{V}=0.075\right)$, we consider this to be a likely RGB, rather than AGB star, noting that the brightest non-variable star lies $>0.4$ mag faintward in $J$ and $K_{S}$.

NGC 1261: The selected tip star lies $18 \operatorname{arcsec}\left(<0.5 R_{\mathrm{hl}}\right)$ from the cluster centre and is therefore a likely member. $H$-band photometry has been taken from 2MASS.

NGC 1851: the brightest candidate tip star in our catalogue, with $\left(J, K_{S}\right)=(9.797,8.704)$ is 5 arcsec from the cluster centre and therefore likely subject to blending. The next best candidate based on $\left(J-K_{S}\right)$ colour is $\mathrm{V} 9$, for which Layden et al. (2010) report $A_{V}=0.43$ and a period of $\sim 141 \mathrm{~d}$. Although this star has a sufficiently small amplitude that we cannot exclude an RGB status, in light of its relatively long period we instead adopt the next faintest candidate, that is $<0.04$ mag fainter in $J$ and $K_{S}$ and not known to be variable (again employing $J, K_{S}$ photometry from our ISPI catalogue and $H$ from 2MASS). This star is also a likely cluster member since it is $23 \operatorname{arcsec}\left(\sim 0.75 R_{\mathrm{hl}}\right)$ from the cluster centre.

NGC 2808: we exclude the brightest candidate TRGB star, variable V45, based on its long period (332 d) and large amplitude $\left(A_{V}=0.8\right)$. The next faintest candidate is also a variable, V31, but we consider this a viable candidate RGB star given the shorter period $(60 \mathrm{~d})$ and smaller amplitude $\left(A_{V}=0.5\right)$. It is almost certainly a member, given its distance from the cluster centre of $\sim 0.9 R_{\mathrm{hl}}$ as well as stellar parameters and abundances from high-resolution spectroscopy (Carretta 2006).

NGC 4833: we have rejected the two brightest candidate TRGB stars based on their blue colours, although one of these is variable V9 with a period of $87.7 \mathrm{~d}$ and unknown amplitude, and therefore an RGB status cannot be completely ruled out. The next best candidate, 2MASS J12594940-7054147, has photometry in excellent agreement with the location of the upper RGB and is located at $<0.9 R_{\mathrm{hl}}$ from the cluster centre. However, if either of the aforementioned brighter, bluer candidates is an RGB member, the TRGB magnitudes would move brightward by $<0.16$ and 0.08 mag in $J$ and $K_{S}$, respectively.

NGC 5927: the chosen tip star is $1.08 \operatorname{arcmin}\left(\sim R_{\mathrm{hl}}\right)$ from the cluster centre, so a likely member, although given its slightly blue colour and the field contamination in the direction of this cluster, its membership remains to be confirmed.

NGC 6304: the brightest candidate, 2MASS J171452742927586 , has not been chosen as its relatively large distance from the cluster centre $\left(>3.1 R_{\mathrm{hl}}\right)$ implies that it may not be a member in light of the field contamination towards this cluster. We choose the next brightest candidate, that is 0.25 and 0.06 mag fainter in $J$ and $K_{S}$, although at $1.35 R_{\mathrm{hl}}$ from the cluster centre, its membership also remains to be confirmed. This is variable V15, listed as NSV 08361 in Samus et al. (2009), although the Clement et al. (2001) catalogue states that it may be constant based on the study of Hartwick, Barlow \& Hesser (1981). The next two fainter candidates are well within $R_{\mathrm{hl}}$, and if our chosen candidate turns out to be a nonmember, a confirmation of membership for these two latter can- didates would move the TRGB as much as $\sim 0.12$ mag faintward in $J$ and $K_{S}$.

NGC 6496: we exclude the brightest candidate, that is variable V7 in the Clement et al. (2001) catalogue, as it lies near the cluster tidal radius and has mean optical colours and magnitudes inconsistent with RGB membership (Abbas et al. 2015), in addition to a relatively blue colour from 2MASS. Our chosen TRGB star is the brightest of the next three best candidates, that are all variables with mean optical photometry from Abbas et al. (2015) as well as single-epoch photometry from 2MASS placing them on the cluster RGB. However, we note that our selected TRGB candidate V4 has $\mathrm{Xflg}=2$ in 2 MASS.

NGC 6584: the chosen tip star is $>0.6 R_{\mathrm{hl}}$ from the cluster centre $\left(\sim 0.6 R_{\mathrm{t}}\right)$ and therefore its membership status should be confirmed. If a non-member, the two next best candidates are both within the cluster $R_{\mathrm{hl}}$ and would imply a faintward shift in the TRGB magnitude of 0.14 and 0.24 mag in $J$ and $K_{S}$, respectively.

NGC 7099 (M 30): we select the same TRGB star as Valenti et al. (2004b), that is within the cluster $R_{\mathrm{hl}}$. While there are two brighter candidates within the cluster tidal radius, they lie far $\left(>12 R_{\mathrm{hl}}\right)$ from the cluster centre, and in both cases, their foreground nature is confirmed by spectroscopic abundances (Kordopatis et al. 2013) and distances (Ammons et al. 2006). Additional clusters from Valenti et al. (2004b); V10: NGC 5904 (M 5): we adopt the same TRGB star as Valenti et al. (2004b), variable V50. Arellano Ferro et al. (2015) have determined a period of $107.6 \mathrm{~d}$, but given its small amplitude evident in their Fig. $3\left(A_{I} \sim 0.3\right)$, its location in optical and near-IR CMDs, and its proximity to the cluster $\left(<1.1 R_{\mathrm{hl}}\right)$ this star is a likely member. However, if not a member, the next brightest candidate, that lies with $R_{\mathrm{hl}}$, would move the TRGB magnitude 0.14 and $0.1 \mathrm{mag}$ faintward in $J$ and $K_{S}$.

NGC 6205 (M 13): the tip star we adopt, the same employed by Valenti et al. (2004b), is V24. Given its period of $45.34 \mathrm{~d}$ and small amplitude $\left(A_{V}=0.24\right)$ from the Clement et al. (2001) catalogue, its pulsational properties may be consistent with an RGB rather than AGB status. Alternatively, a significant $(\Delta Y>0.05)$ helium enhancement in this cluster (e.g. Caloi \& D'Antona 2005; Johnson \& Pilachowski 2012; Dalessandro et al. 2013; VandenBerg et al. 2013) could substantially affect the location of both the RGB bump and tip.

NGC 6388: the previously employed TRGB candidate (V10) is now known to be V3, a Mira variable (Sloan et al. 2010). Meanwhile, $\mathrm{V} 12$ (= star 1 in the catalogue of V10) is a brighter candidate, although it is listed as a long-period variable with an amplitude of $A_{V}=0.6$, leaving its evolutionary status uncertain. However, in the Washington CMD of Hughes et al. (2007), the location of this star appears inconsistent with the location of the RGB, so we discard it. As there are no other viable TRGB candidates within $\sim 12 R_{\mathrm{hl}}$, we therefore select the next faintest candidate, that has Washington photometry placing it on the RGB, and is not a known variable. This represents a faintward shift of $\sim 0.1$ mag in $K_{S}$ and a brightward shift of $>0.2$ mag in $H$ from the V10 candidate, and these shifts would be even larger (in absolute value) if V12 were to be confirmed as an RGB, rather than AGB, member.

NGC 6752: we select the same tip star as Valenti et al. (2004b), 2MASS J19110813-6001517, that is $3.43 \operatorname{arcmin}\left(\sim 1.8 R_{\mathrm{hl}}\right)$ from the cluster centre. Although there are several brighter candidate tip stars, they lie significantly farther $\left(\gtrsim 5 R_{\mathrm{hl}}\right)$ from the cluster centre, and unfortunately none of these could be matched to recent proper motion or radial velocity studies.

This paper has been typeset from a $\mathrm{T}_{\mathrm{E}} \mathrm{X} / \mathrm{L} \mathrm{T}_{\mathrm{E}} \mathrm{X}$ file prepared by the author. 